\title{
Fe(II) Hydride Complexes for the Homogeneous Dehydrocoupling of Hydrazine Borane: Catalytic Mechanism via DFT Calculations and Detailed Spectroscopic Characterization
}

\author{
Robert Knitsch,$^{\dagger \mathrm{a}}$ Delong Han,${ }^{\dagger \mathrm{b}}$ Felix Anke, ${ }^{\mathrm{b}}$ Lukas Ibing ${ }^{\mathrm{a}, \mathrm{c}}$, Haijun Jiao ${ }^{\mathrm{b}}$, Michael Ryan Hansen ${ }^{*^{\mathrm{a}}}$ and \\ Torsten Beweries ${ }^{*}$ \\ ${ }^{a}$ Institute for Physical Chemistry, Westfälische Wilhelms-Universität Münster, Corrensstr. 28/30, 48149 \\ Münster, Germany. \\ ${ }^{\text {b }}$ Leibniz-Institut für Katalyse an der Universität Rostock e.V., Albert-Einstein-Str. 29a, 18059 Rostock, \\ Germany. \\ ${ }^{\mathrm{c}}$ Institute for Physical Chemistry, MEET Battery Research Center, Westfälische Wilhelms-Universität \\ Münster, Corrensstr. 46, 48149 Münster, Germany. \\ mhansen@uni-muenster.de, $\underline{\text { torsten.beweries@ catalysis.de }}$
}

\section{Table of contents}

S2 Experimental details

S4 Volumetric data

S6 NMR spectra

S15 GC analysis

S16 Computational Details

S43 Solid-state NMR experiments

S49 XPS analysis

S50 Raman spectroscopy

S51 Validation of the combined NMR and DFT approach

S54 References 


\section{Experimental details}

General: All manipulations were carried out in an oxygen- and moisture-free argon atmosphere using standard Schlenk- and glovebox techniques. All solvents were dispensed from a solvent purification system (PureSolv, Innovative Technology) or dried over sodium/benzophenone and freshly distilled prior to use. Complexes $\mathbf{1}-\mathbf{B H}_{\mathbf{3}}{ }^{1}$ and $\mathbf{2}^{2}$ as well as hydrazine borane ${ }^{3}$ were prepared according to published procedures. $\left(\mathrm{N}_{2} \mathrm{H}_{5}\right) \mathrm{SO}_{4}, \mathrm{HN}\left(\mathrm{CH}_{2} \mathrm{CH}_{2} \mathrm{P}_{2} \mathrm{Pr}_{2}\right)_{2}$ (10\% in THF), $\mathrm{FeBr}_{2}, \mathrm{Fe}$ powder, $\mathrm{KO} t \mathrm{Bu}, \mathrm{NaHBEt}_{3}$ and $\mathrm{NaBH}_{4}$ were purchased from Sigma Aldrich and used as received.

NMR spectra were recorded on a Bruker AV 300 or AV 400 spectrometer. All ${ }^{1} \mathrm{H}$ NMR spectra are referenced using the chemical shifts of residual protio solvent resonances (THF- $d_{8}: \delta H 1.73,3.58, \delta \mathrm{C}$ 25.4, 67.6). Chemical shifts are reported in ppm $(\delta)$ relative to tetramethylsilane. The ${ }^{31} \mathrm{P}\left\{{ }^{1} \mathrm{H}\right\} \mathrm{NMR}$ spectra were referenced to external $\mathrm{H}_{3} \mathrm{PO}_{4}$, the ${ }^{11} \mathrm{~B}$ NMR spectra were referenced to $\mathrm{BF}_{3} \cdot \mathrm{Et}_{2} \mathrm{O}$ in chloroform. Gas chromatography was performed using an Agilent Technologies 7890A gas chromatograph with a 60/80 Carboxen 1000 (Supelco) column and a TCD was used to qualify the gas.

\section{Catalytic studies:}

General procedure for dehydrogenation experiments: Hydrazine borane (HB, $50 \mathrm{mg}, 1.09 \mathrm{mmol})$ and the corresponding Fe catalyst $(0.5,1.0,2.0$, or $5.0 \mathrm{~mol} \%$, respectively) were weighed in the glovebox and transferred to a three-necked dehydrogenation vessel and a Schlenk flask, respectively. Then, the HB containing dehydrogenation flask was connected to the gas buret and subsequently equipped with a dropping funnel with pressure compensation under Ar atmosphere. The automatic gas buret was initialised and $\mathrm{HB}$ and Fe catalyst were dissolved in $5 \mathrm{~mL}$ of THF, respectively. The catalyst solution was transferred to the dropping funnel, the data acquisition (values read automatically each 5 seconds) was started and the catalyst solution was quickly added to the HB solution via the dropping funnel. After completion of the dehydrogenation reaction, a gas sample was taken and analysed by TCD-GC. The reaction solution was filtered and the BN residue as well as the filtrate were further analysed. Details of the experimental setup used for volumetric analysis were published before. ${ }^{4,5}$

\section{Recycling of Fe catalysts:}

Fe catalysts $\left(5 \mathrm{~mol} \%\right.$ ) were dissolved in $2 \mathrm{~mL}$ of $\mathrm{THF}$ in a reaction vessel at $25{ }^{\circ} \mathrm{C}, 40 \mathrm{mg} \mathrm{HB}$ was dissolved in $8 \mathrm{~mL}$ of THF and was transferred into a dropping funnel connected to the reaction vessel. When the reaction started, $2 \mathrm{~mL}$ of the $\mathbf{H B} / \mathrm{THF}$ solution was added to the reactor. For complex $\mathbf{1 - B \mathbf { H } _ { 3 }}$ after release of approximately 0.7 equivalents of $\mathrm{H}_{2}, 2 \mathrm{~mL}$ of fresh HB/THF solution was added.

For complex 2 after release of approximately 2.2 equivalents of $\mathrm{H}_{2}, 2 \mathrm{~mL}$ of fresh HB/THF solution was added. The procedure was repeated three times. Catalysts were completely consumed after the fourth addition.

\section{$\mathrm{PMe}_{3}$ poisoning experiments:}

To verify the homogeneous nature of the catalyst $\mathrm{PMe}_{3}$ poisoning experiments were done. For a reaction using catalyst $\mathbf{1 - B H _ { 3 }}\left(25^{\circ} \mathrm{C}, 10 \mathrm{~mL}\right.$ THF, 2 mol\% $\left.\mathbf{1}-\mathbf{B H}_{3}\right) \mathrm{PMe}_{3}$ was added into a reaction system $(5 \mu 1$, $0.52 \mathrm{M}$ in THF, $0.02 \mathrm{~mol} \%$ loading) after release of approximately 0.3 equivalents of $\mathrm{H}_{2}$.

For a reaction using catalyst $2\left(25{ }^{\circ} \mathrm{C}, 10 \mathrm{~mL}\right.$ THF, 2 mol\% 2) $\mathrm{PMe}_{3}$ was added into a reaction system ( $5 \mu 1,0.52 \mathrm{M}$ in THF, $0.02 \mathrm{~mol} \%$ loading) after release of approximately 0.4 equivalents of $\mathrm{H}_{2}$. In both cases no influence on the activity of the systems was observed.

\section{NMR studies:}


For the NMR experiments at room temperature, reactions were done in an open system, i.e. in a Schlenk flask connected to an oil bubbler using the same parameters as used in volumetric experiments (vide supra), using THF- $d_{8}$ as solvent. An aliquot was taken at times reported and the sample was analyzed by NMR spectroscopy.

For the VT NMR experiments (closed system) samples were freshly prepared prior to introduction into the NMR probe head. For this, pre-cooled solvent was transferred into a Young NMR tube containing the catalyst and $\mathrm{HB}$ as solids at $-78{ }^{\circ} \mathrm{C}$. The tube was sealed and the Ar atmosphere was removed at $-78{ }^{\circ} \mathrm{C}$ to allow for build-up of hydrogen pressure during reaction. After immediate introduction into the NMR spectrometer, the temperature of the sample was gradually raised to room temperature with spectra being recorded at temperatures reported. 


\section{Volumetric data}

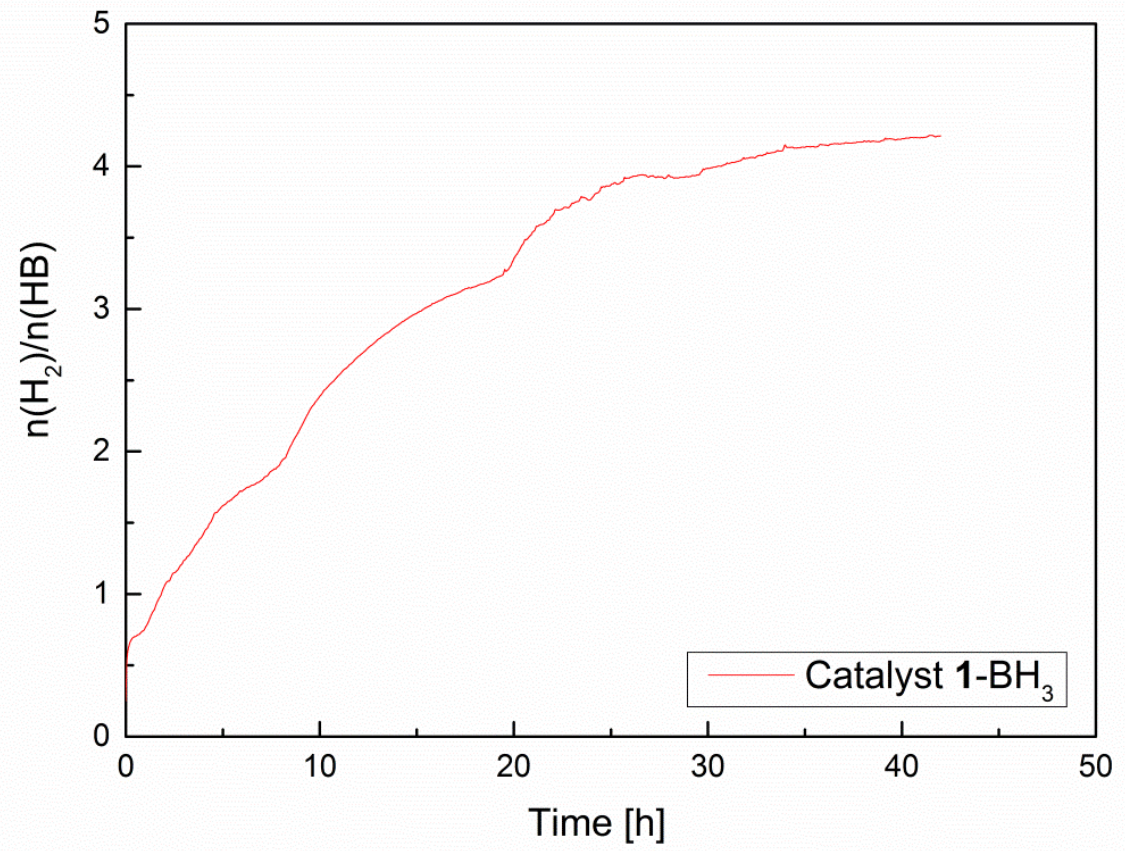

Figure S1. Volumetric data of recycling experiments using catalyst $\mathbf{1}-\mathbf{B H}_{3}(5 \mathrm{~mol} \%$ initial catalyst concentration, $25^{\circ} \mathrm{C}$, THF). Fresh solutions of HB were added at approximately $0.7,1.9,3.1$, and 3.9 equivalents of $\mathrm{H}_{2}$ released.

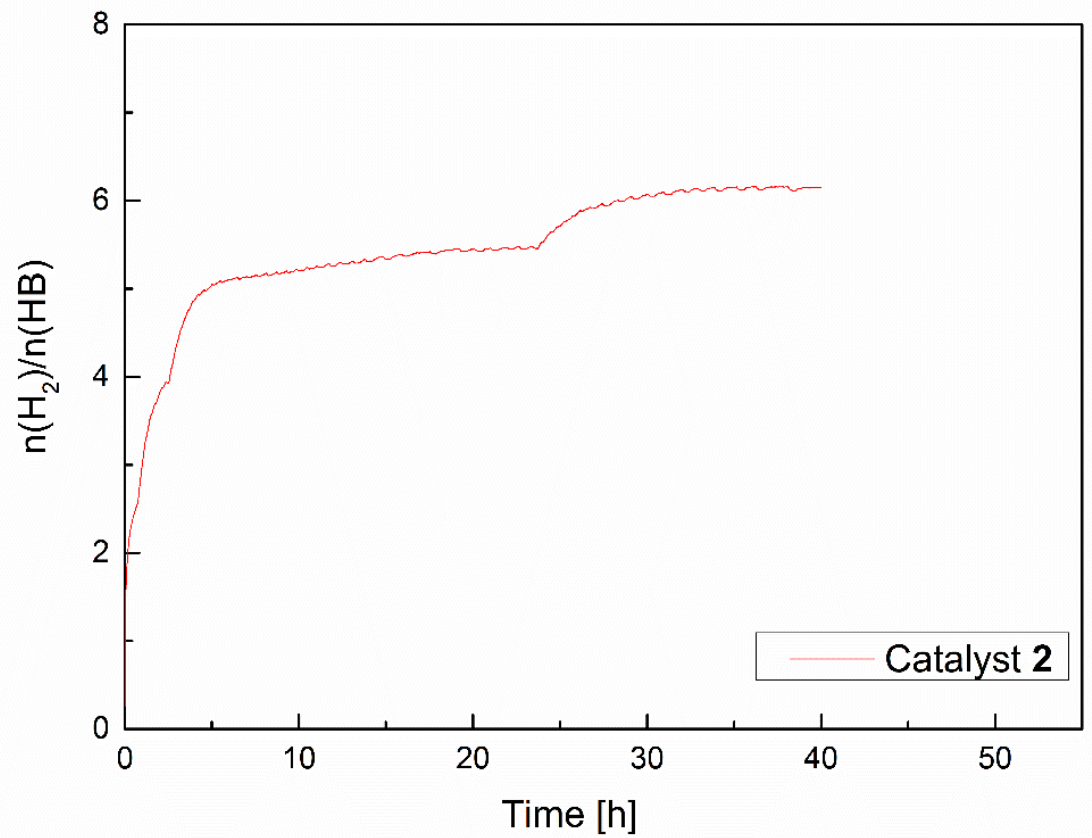

Figure S2 Volumetric data of recycling experiments using catalyst 2 (5 mol\% initial catalyst concentration, $25^{\circ} \mathrm{C}$, THF). Fresh solutions of $\mathrm{HB}$ were added at approximately 2.2, 4.0, and 5.2 equivalents of $\mathrm{H}_{2}$ released. 


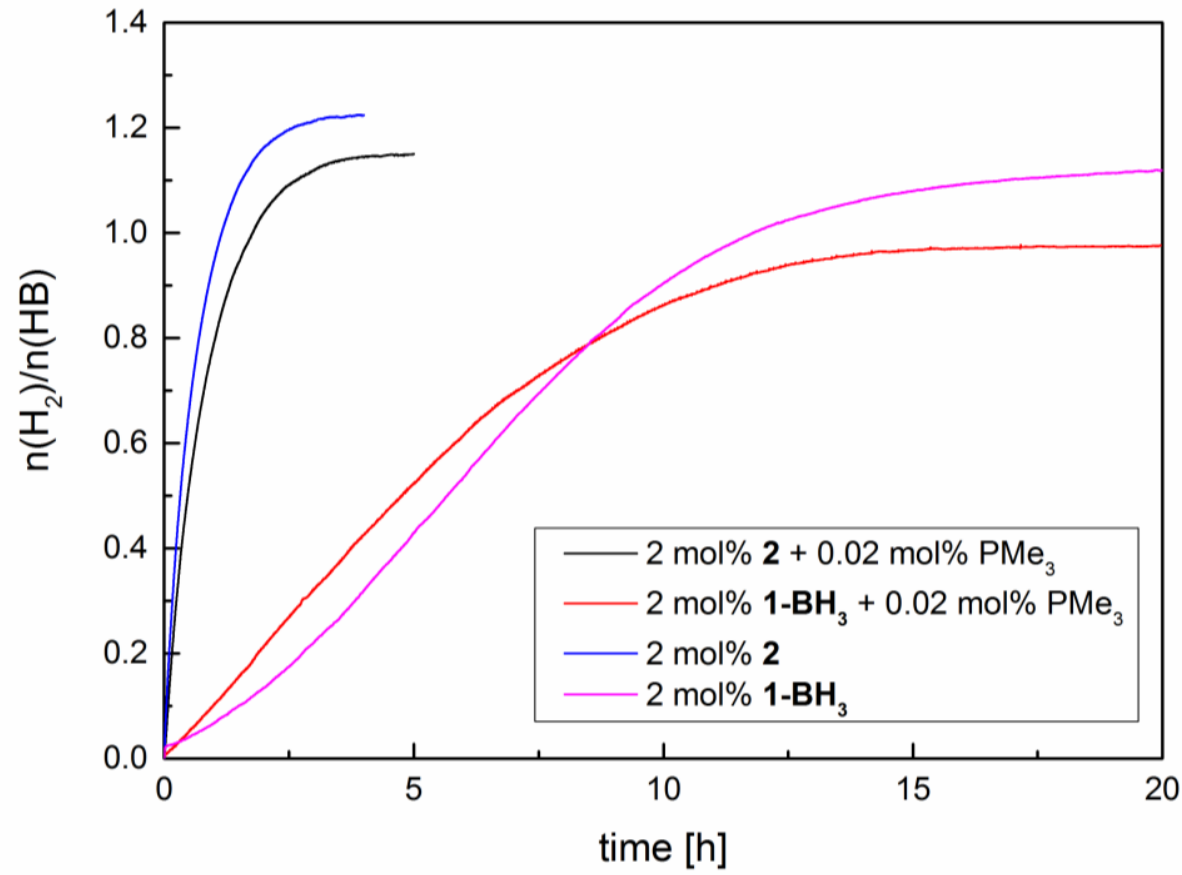

Figure S3. Volumetric data of $\mathrm{PMe}_{3}$ poisoning experiments. For both catalysts $\mathrm{PMe}_{3}$ was added after release of approximately 0.3-0.4 equivalents of hydrogen.

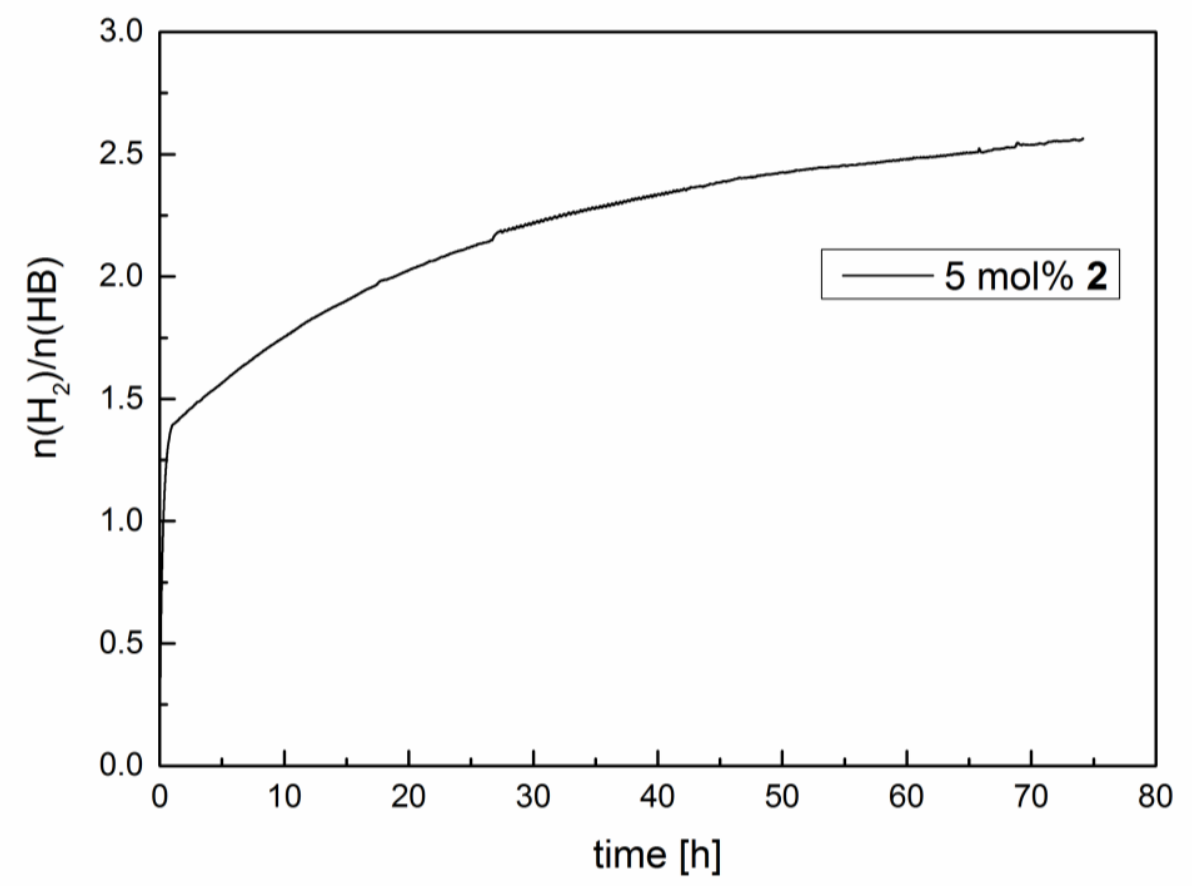

Figure S4. Volumetric curve illustrating a maximum release of 2.56 equivalents of $\mathrm{H}_{2}$ using $5 \mathrm{~mol} \%$ of catalyst 2 (THF, room temperature). 


\section{In situ NMR spectra}

\section{Catalyst 1- $\mathrm{BH}_{3}-$ room temperature (open system)}

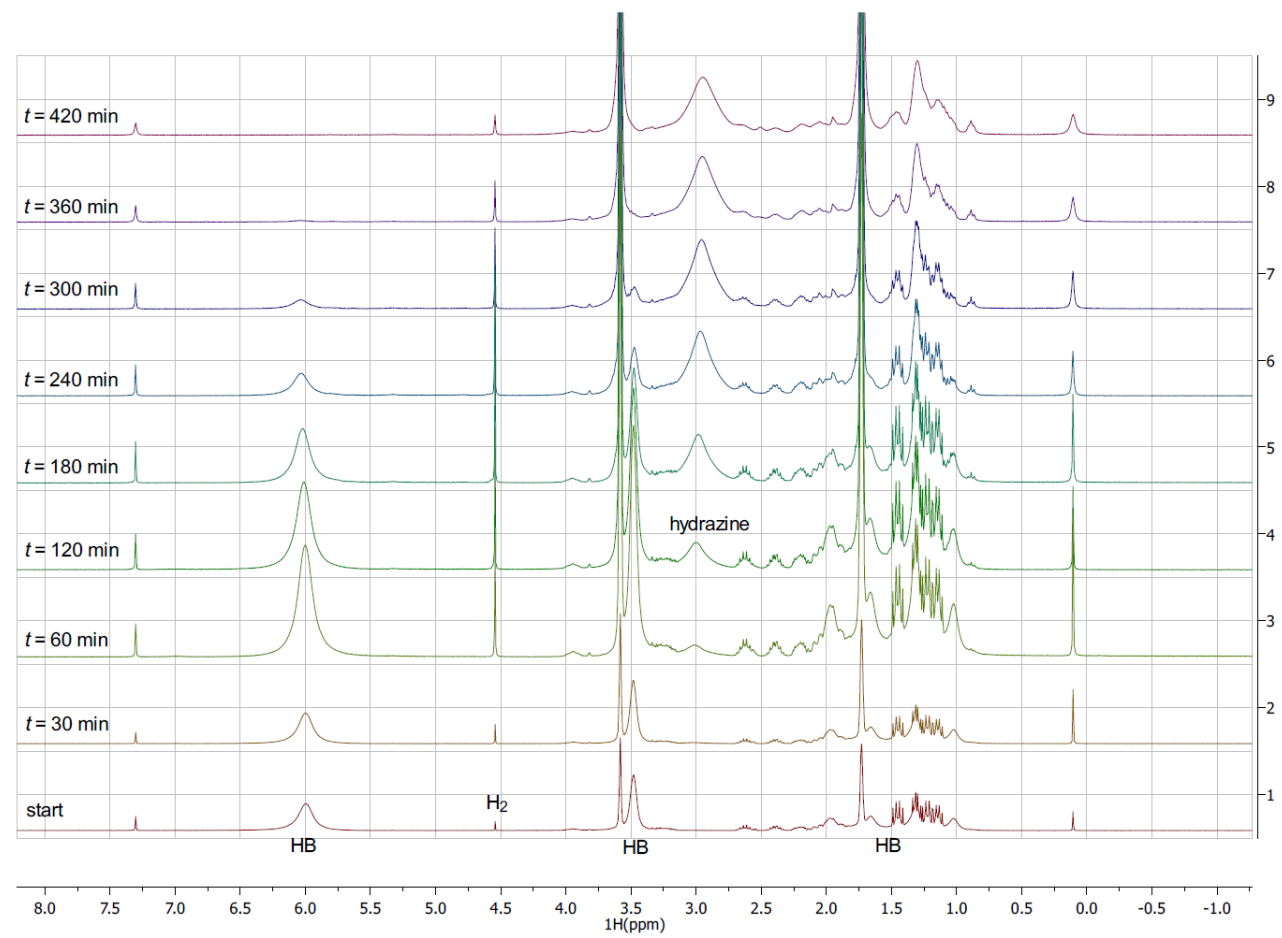

Figure S5. Downfield region of ${ }^{1} \mathrm{H}$ NMR spectra of $\mathrm{HB}$ dehydrogenation using $5 \mathrm{~mol} \% \mathbf{1 - B H _ { 3 }}$ as the catalyst (300 MHz, THF- $\left.d_{8}, 297 \mathrm{~K}\right)$.

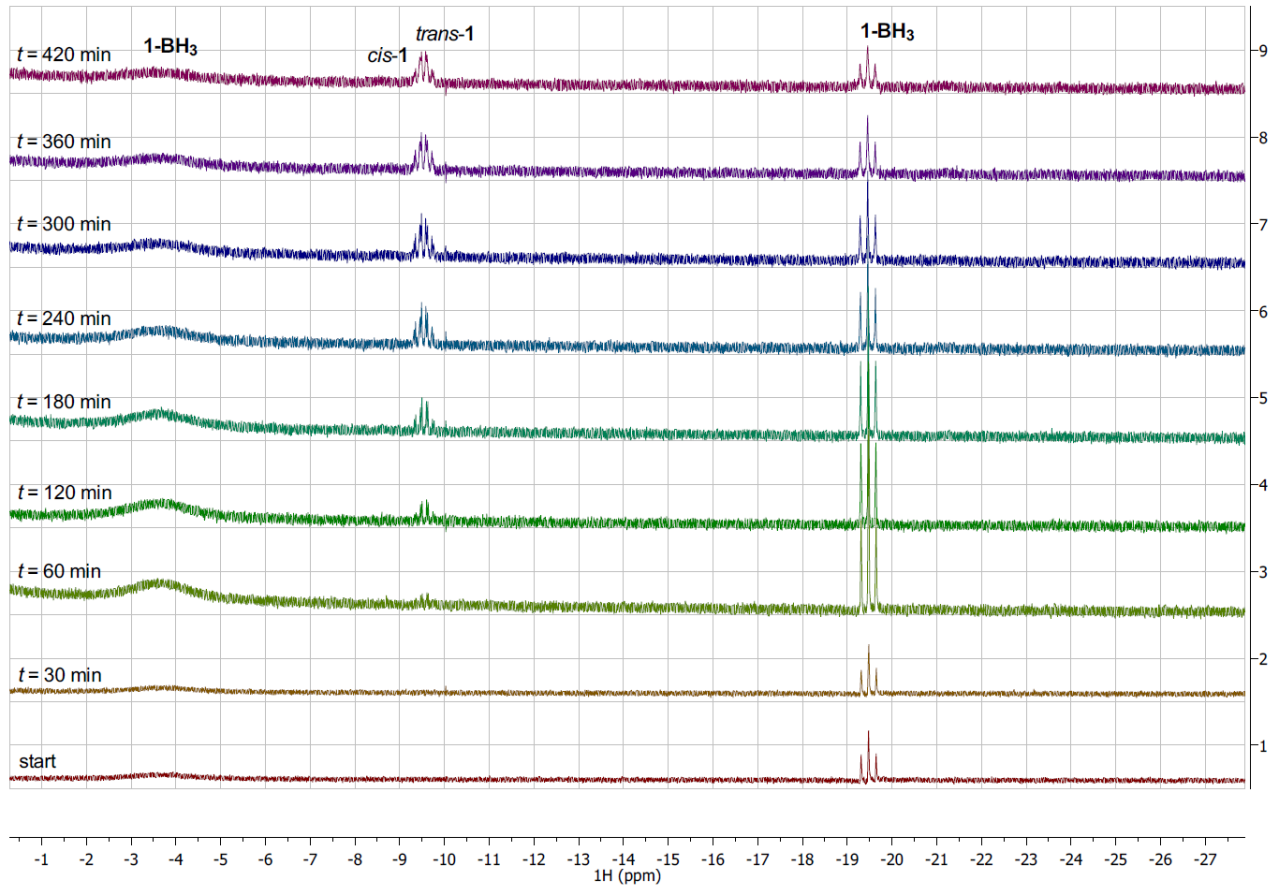

Figure S6. Upfield region of ${ }^{1} \mathrm{H}$ NMR spectra of $\mathrm{HB}$ dehydrogenation using $5 \mathrm{~mol} \% \mathbf{1 - B H}$ as the catalyst (300 MHz, THF- $d_{8}, 297 \mathrm{~K}$ ). 


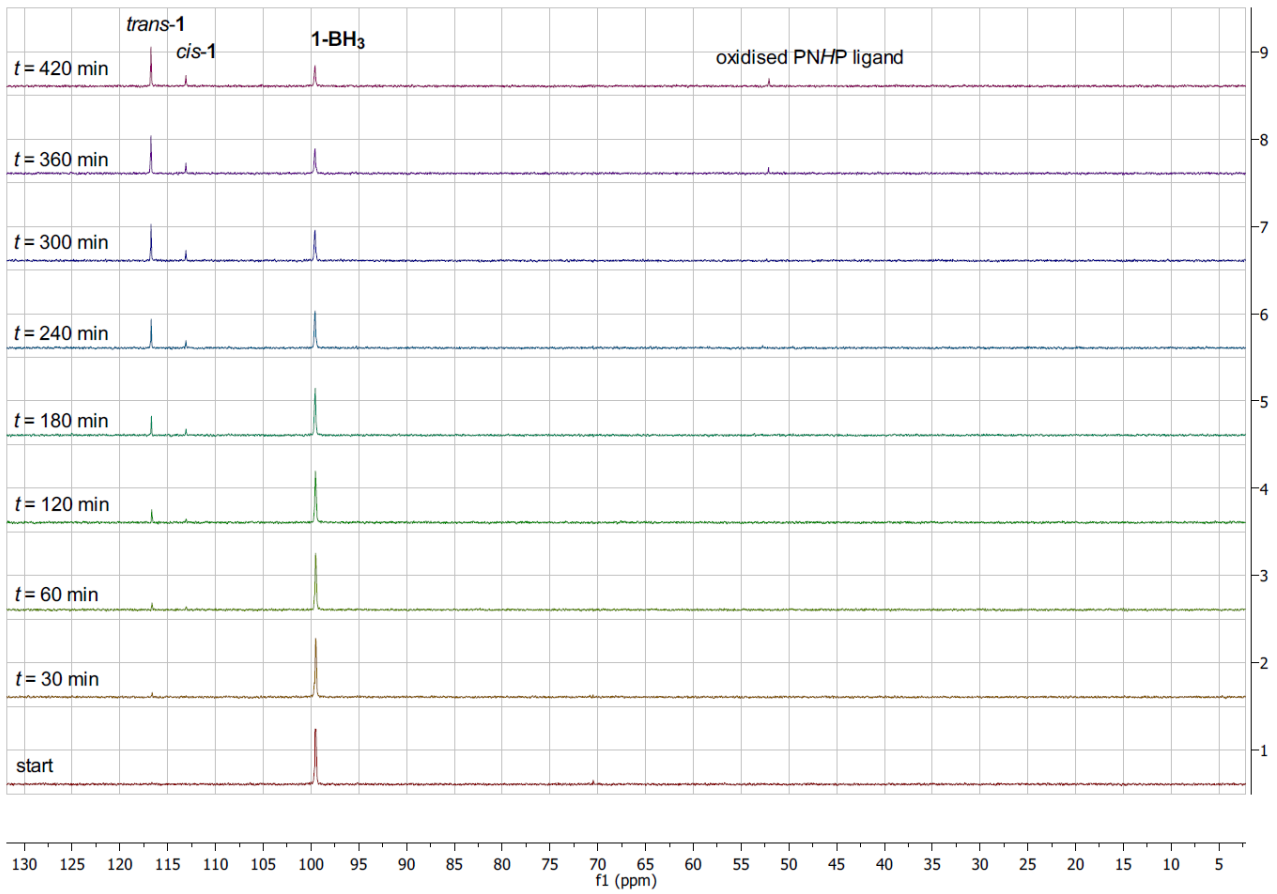

Figure S7. ${ }^{31} \mathrm{P}$ NMR spectra of $\mathrm{HB}$ dehydrogenation using 5 mol\% $\mathbf{1 - B H} \mathbf{B H}_{\mathbf{3}}$ as the catalyst $(121 \mathrm{MHz}$, THF$d_{8}, 297 \mathrm{~K}$ ).

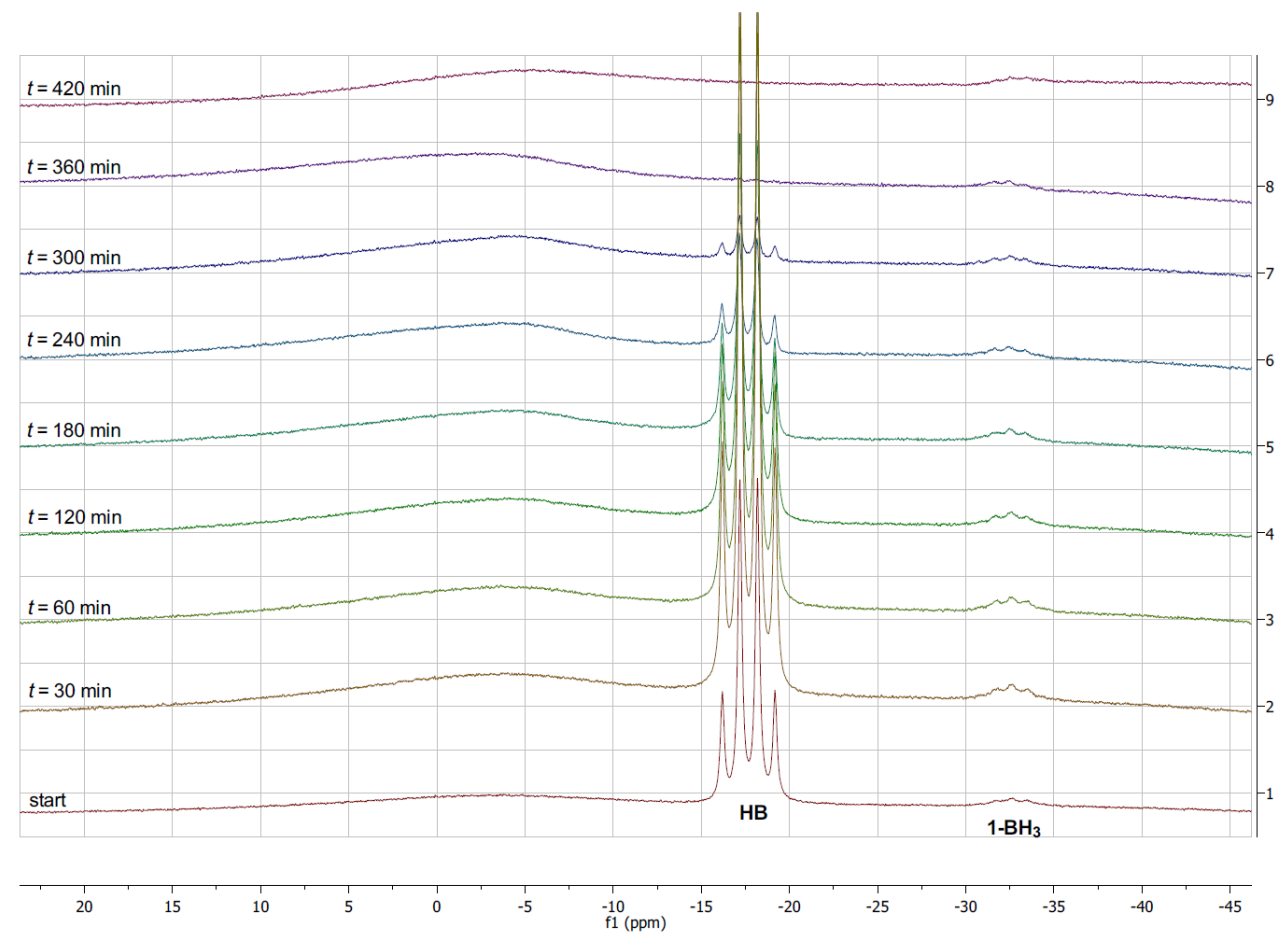

Figure S8. ${ }^{11} \mathrm{~B}$ NMR spectra of $\mathrm{HB}$ dehydrogenation using 5 mol\% $\mathbf{1}-\mathbf{B H}_{\mathbf{3}}$ as the catalyst $(96 \mathrm{MHz}$, THF$\left.d_{8}, 297 \mathrm{~K}\right)$. 


\section{Catalyst 1-BH - VT experiment (closed system)}

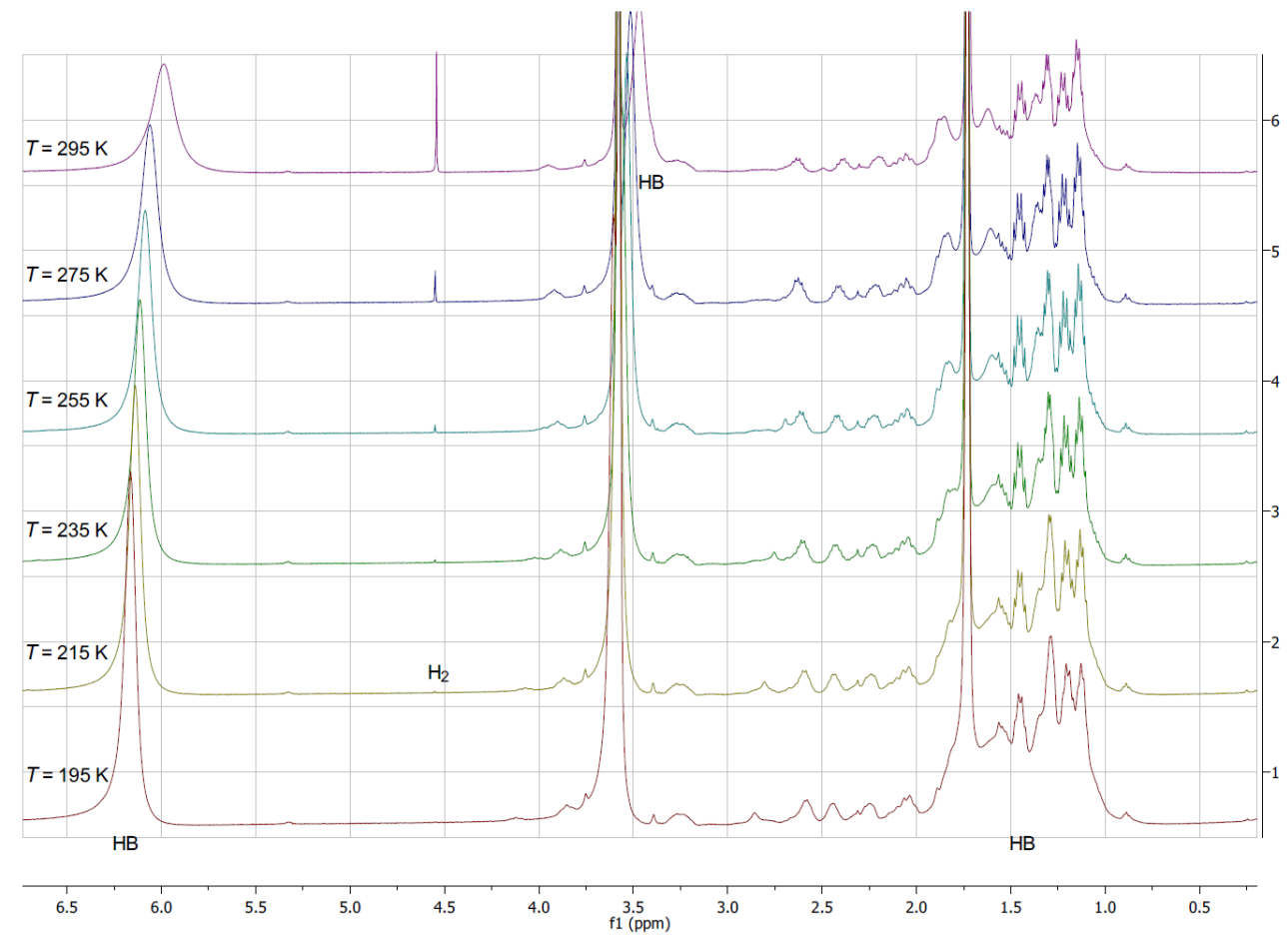

Figure S9. Downfield region of ${ }^{1} \mathrm{H}$ NMR spectra of $\mathrm{HB}$ dehydrogenation using 5 mol\% $\mathbf{1 - B \mathbf { B H } _ { 3 }}$ as the catalyst $\left(300 \mathrm{MHz}, \mathrm{THF}-d_{8}, 297 \mathrm{~K}\right)$.

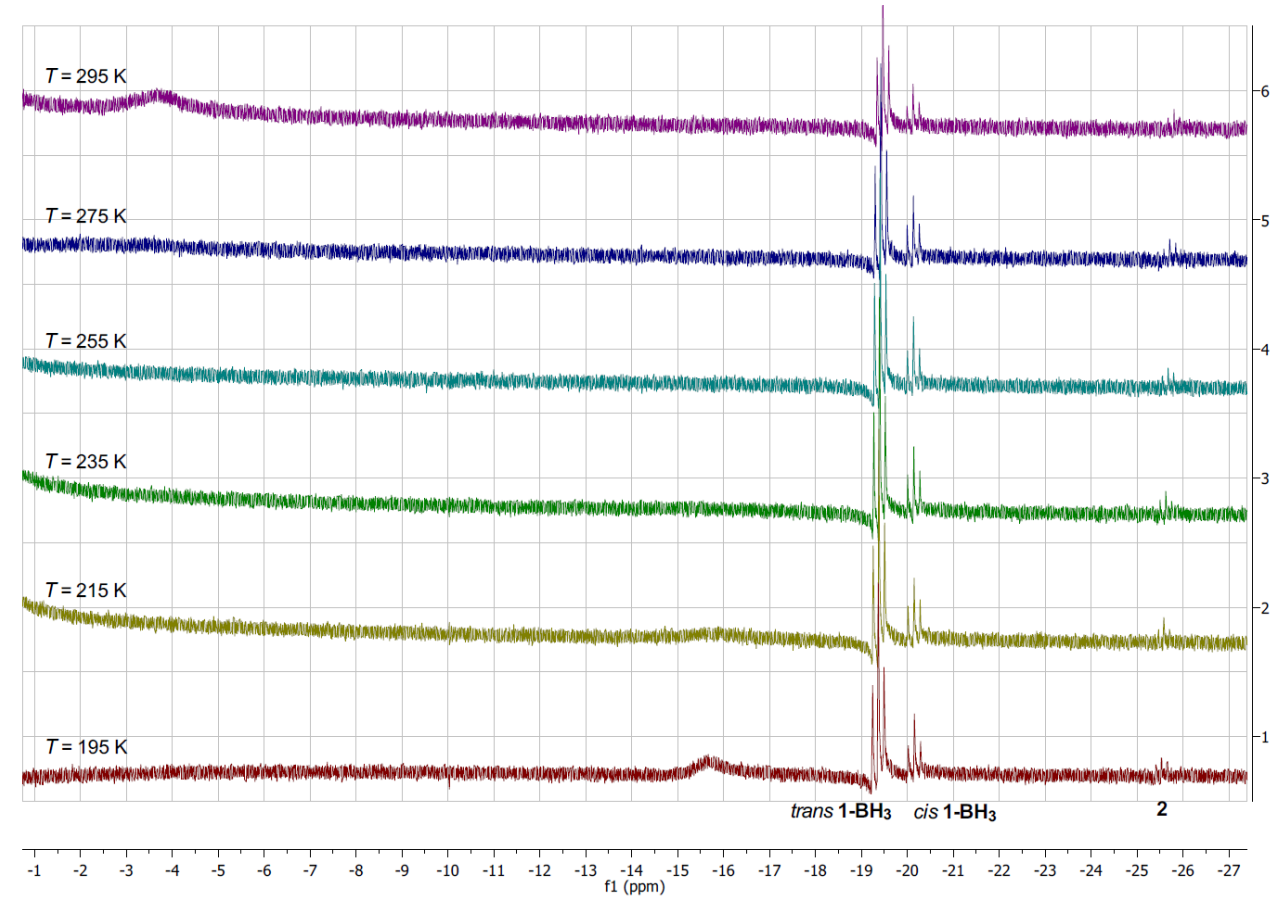

Figure S10. Upfield region of ${ }^{1} \mathrm{H}$ NMR spectra of $\mathrm{HB}$ dehydrogenation using 5 mol\% $\mathbf{1 - B H _ { 3 }}$ as the catalyst (300 MHz, THF- $\left.d_{8}, 297 \mathrm{~K}\right)$. 


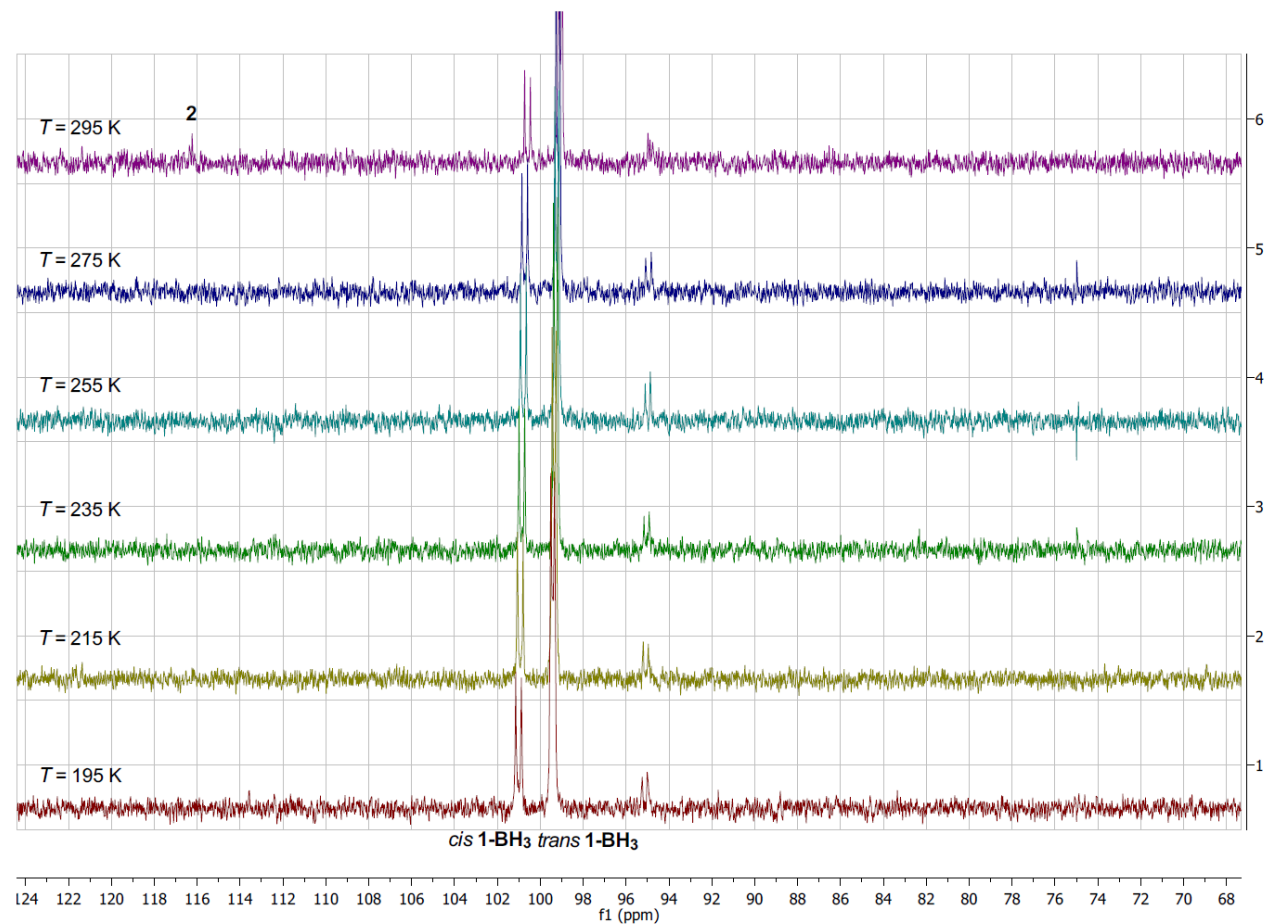

Figure S11. ${ }^{31} \mathrm{P}$ VT-NMR spectrum of HB dehydrogenation using $5 \mathrm{~mol} \% \mathbf{1 - B \mathbf { H } _ { 3 }}$ as the catalyst (121 $\mathrm{MHz}, \mathrm{THF}-d_{8}$ ). 


\section{Catalyst 2 - room temperature (open system)}

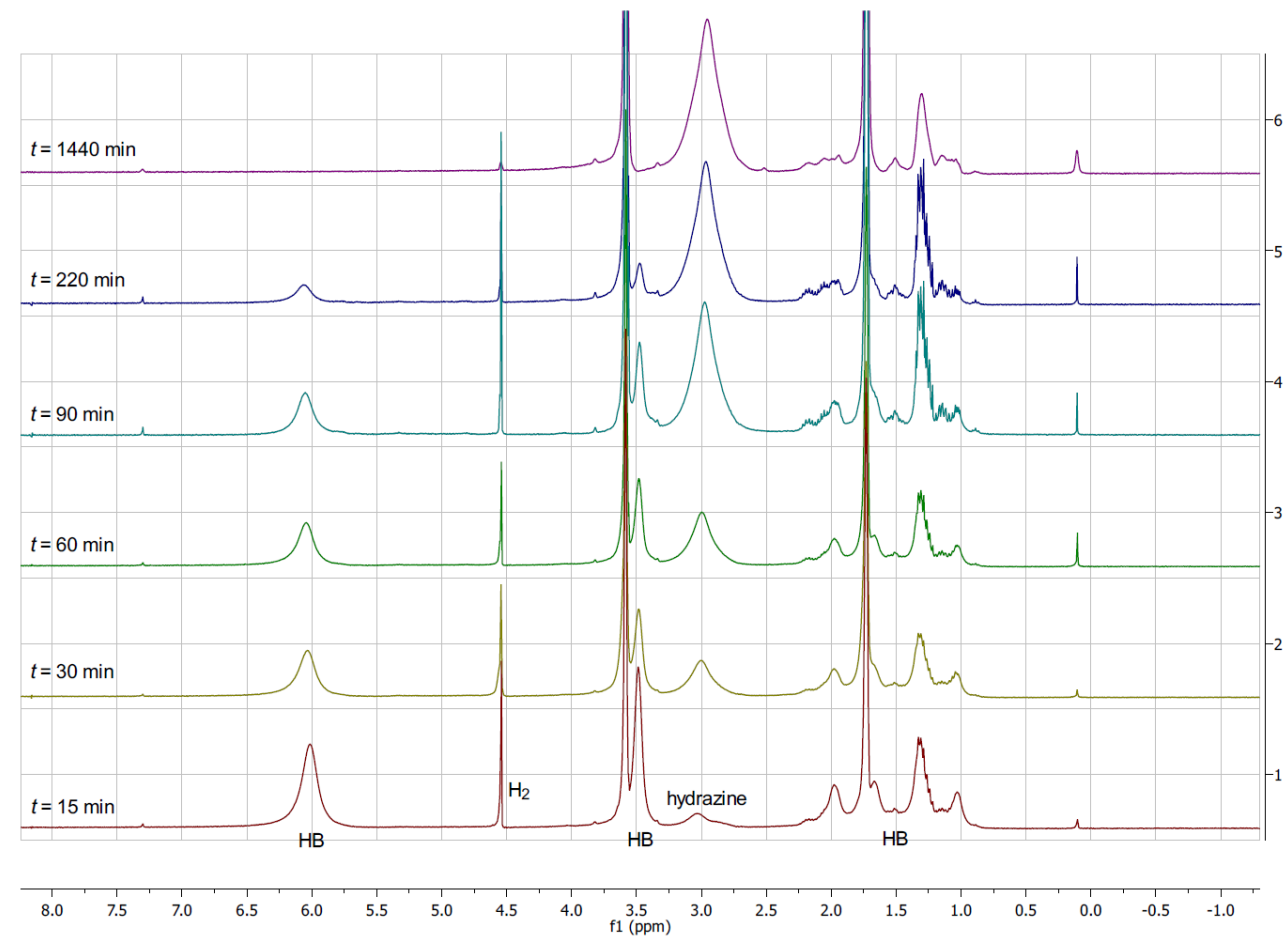

Figure S12. Downfield region of ${ }^{1} \mathrm{H}$ NMR spectra of HB dehydrogenation using 5 mol\% 2 as the catalyst (300 MHz, THF- $\left.d_{8}, 297 \mathrm{~K}\right)$.

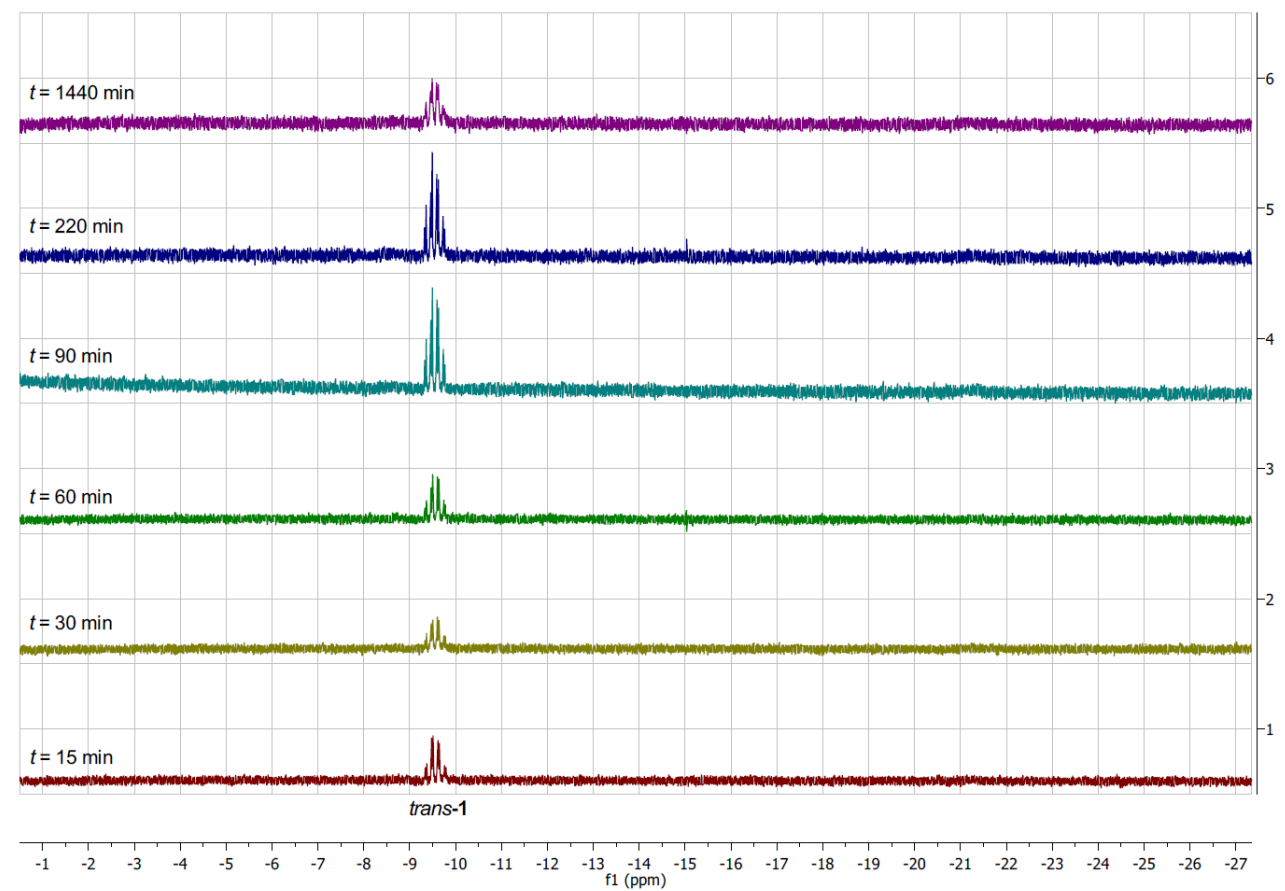

Figure S13 Upfield region of ${ }^{1} \mathrm{H}$ NMR spectra of HB dehydrogenation using $5 \mathrm{~mol} \% \mathbf{2}$ as the catalyst $\left(300 \mathrm{MHz}, \mathrm{THF}-d_{8}, 297 \mathrm{~K}\right)$. 


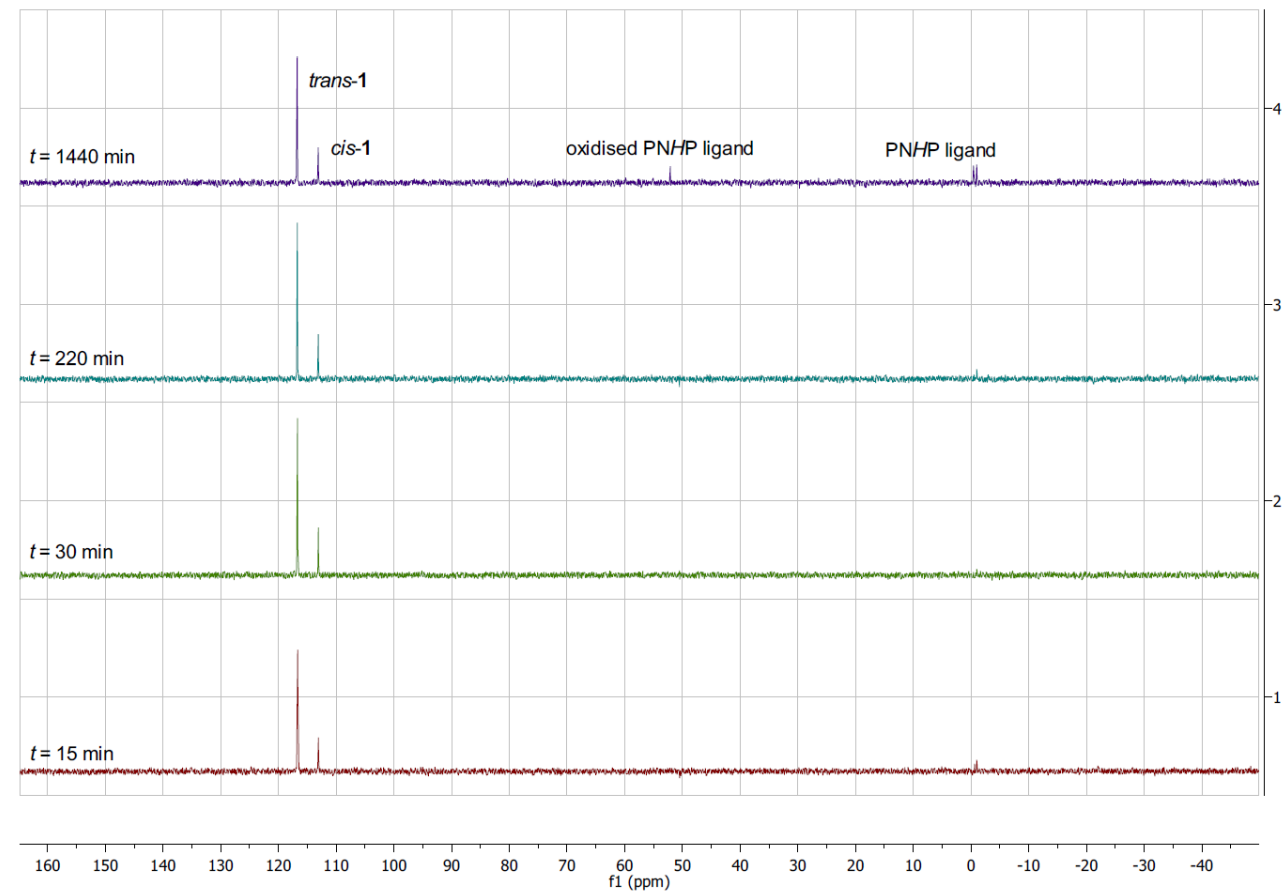

Figure S14. ${ }^{31} \mathrm{P}$ NMR spectrum after HB dehydrogenation using 5 mol\% 2 as the catalyst $(121 \mathrm{MHz}$, THF- $d_{8}, 297 \mathrm{~K}$ ). 


\section{Catalyst 2 - VT experiment (closed system)}

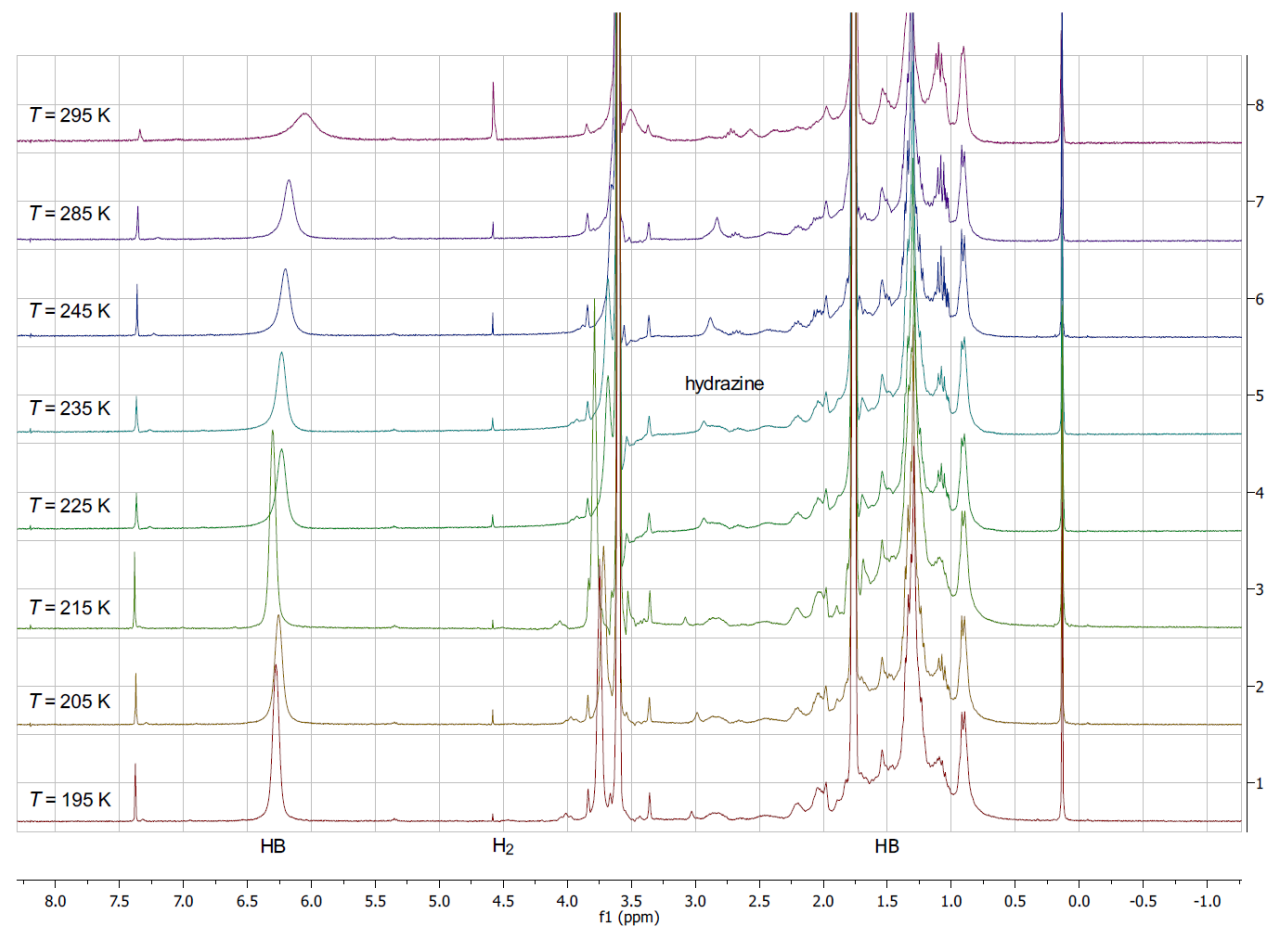

Figure S15. Downfield region of ${ }^{1} \mathrm{H}$ NMR spectra of HB dehydrogenation using 5 mol\% 2 as the catalyst (300 MHz, THF- $d_{8}, 297 \mathrm{~K}$ ).

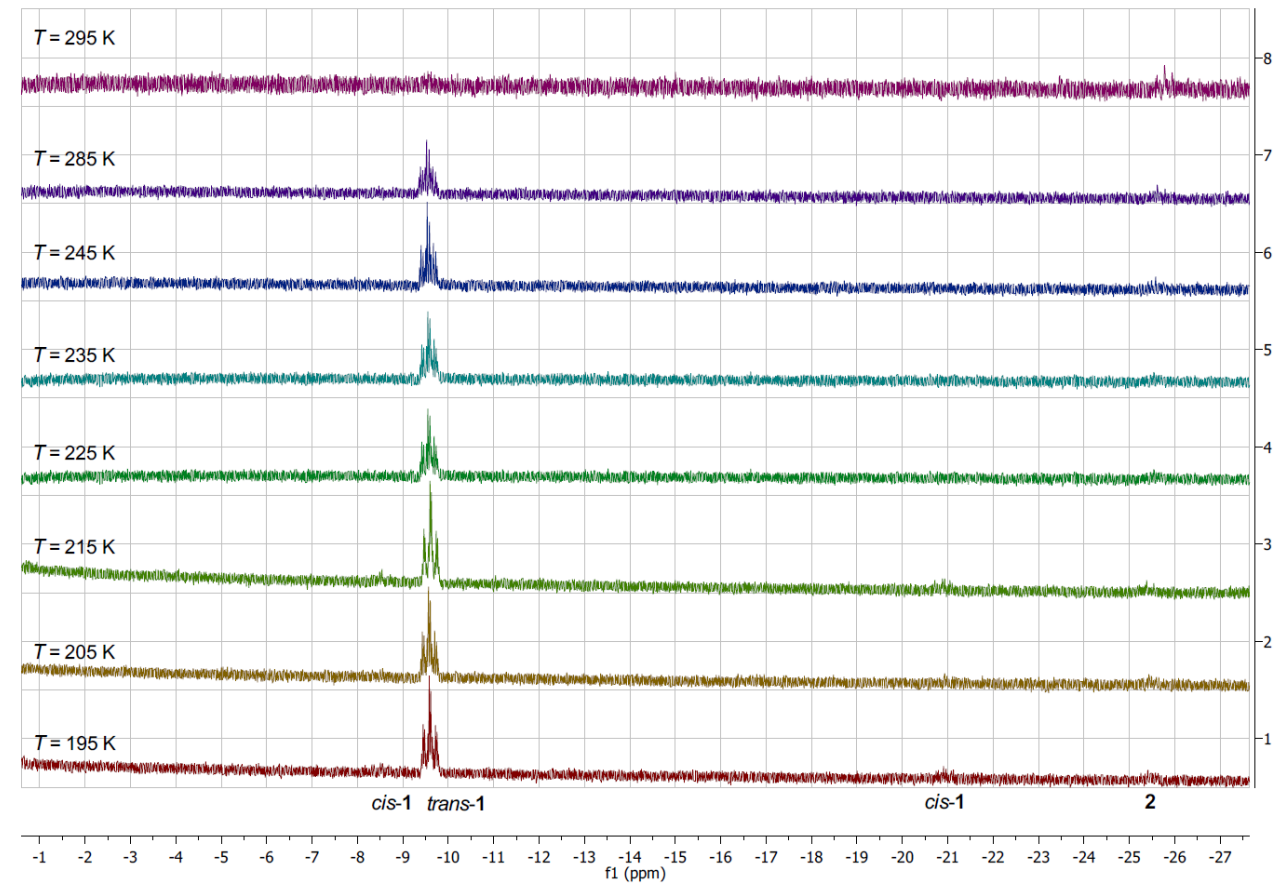

Figure S16. Upfield region of ${ }^{1} \mathrm{H}$ NMR spectra of $\mathrm{HB}$ dehydrogenation using $5 \mathrm{~mol} \% \mathbf{2}$ as the catalyst $\left(300 \mathrm{MHz}, \mathrm{THF}-d_{8}, 297 \mathrm{~K}\right)$. 


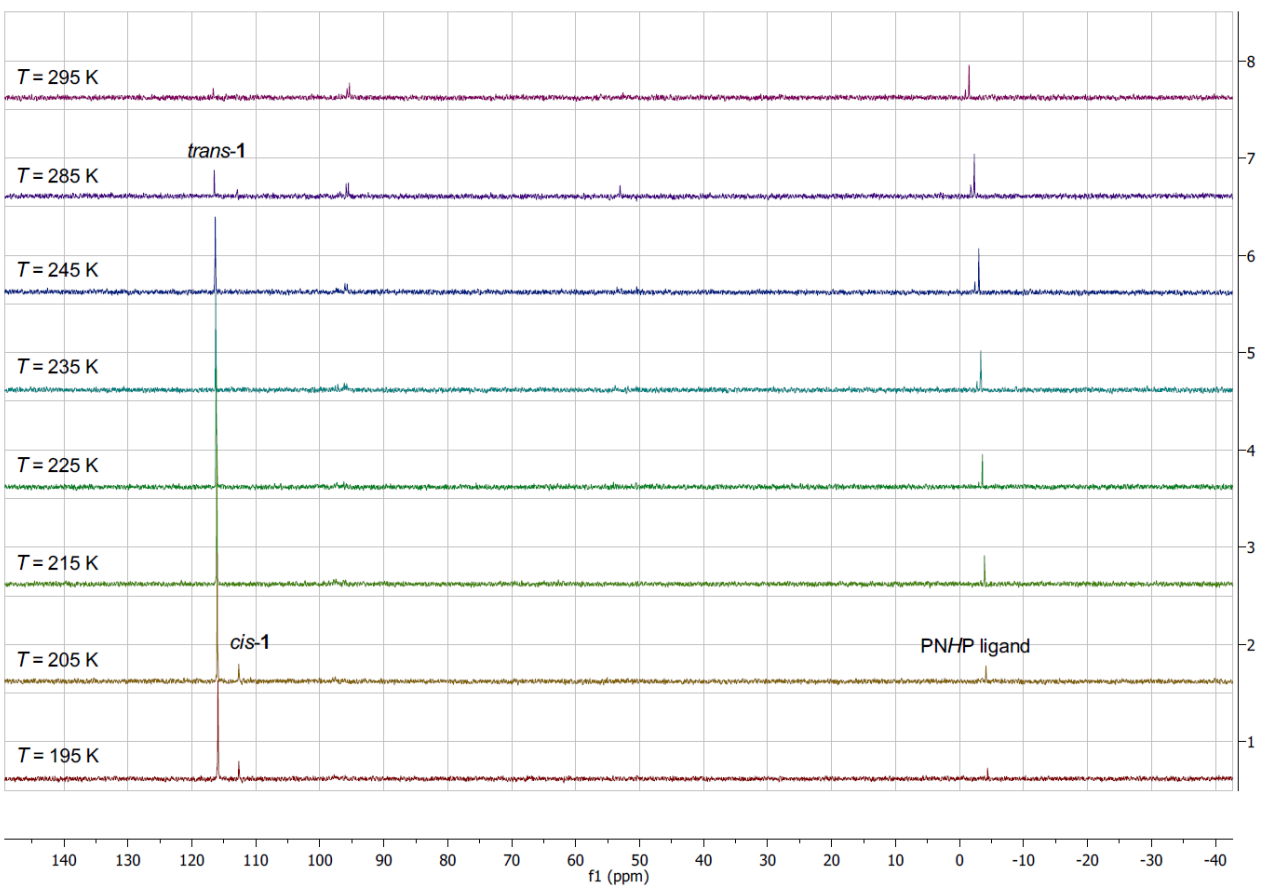

Figure S17. ${ }^{31} \mathrm{P}$ VT-NMR spectrum of HB dehydrogenation using $5 \mathrm{~mol} \% \mathbf{2}$ as the catalyst $(121 \mathrm{MHz}$, THF- $d_{8}$ ). 
Reactions of $\mathrm{HB}$ with equimolar amounts of $1-\mathrm{BH}_{3}$ and 2

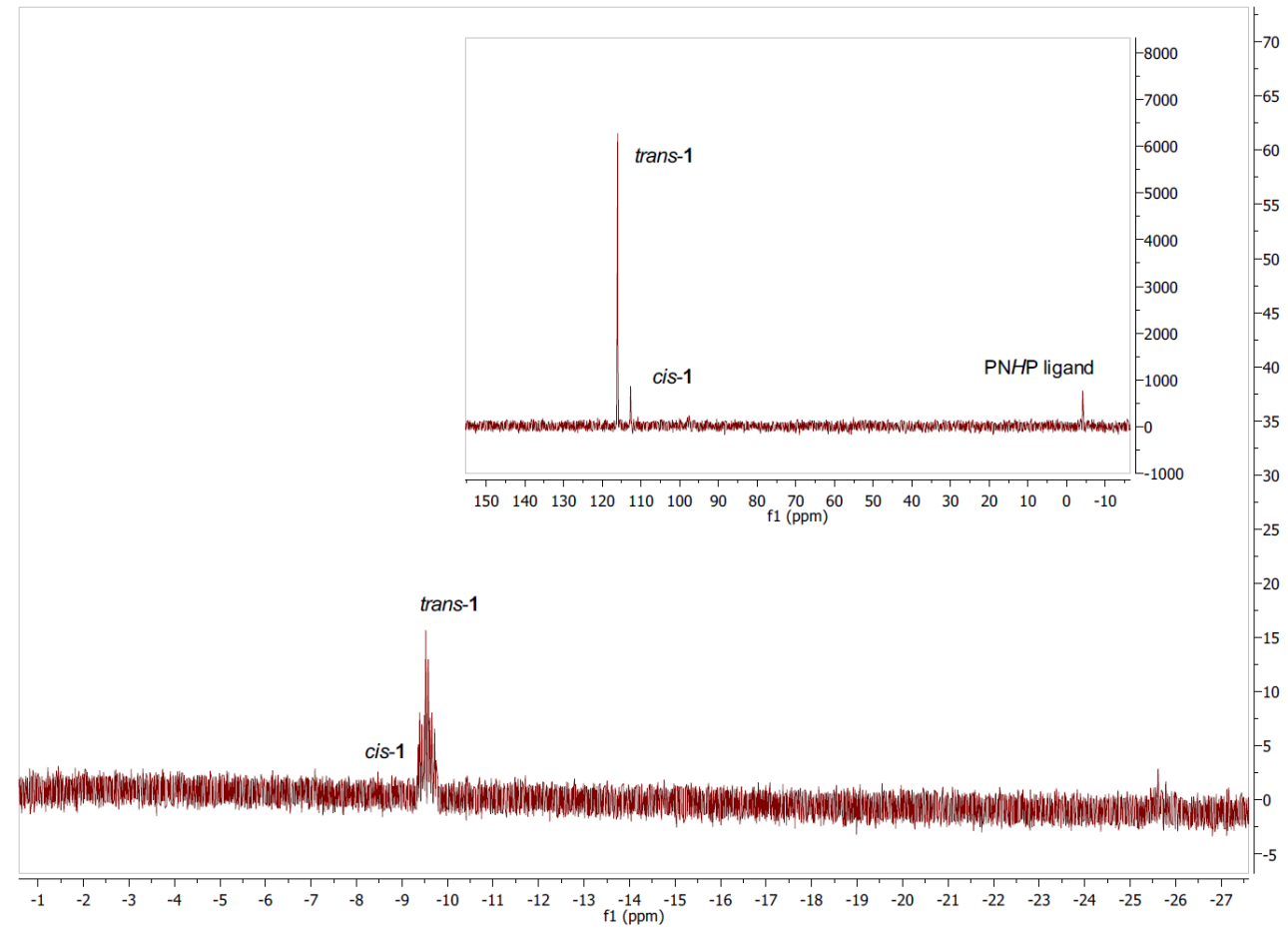

Figure S18. ${ }^{1} \mathrm{H}(300 \mathrm{MHz})$ and ${ }^{31} \mathrm{P}$ NMR (inset, $121 \mathrm{MHz}$ ) spectra of a reaction of $\mathrm{HB}$ with $100 \mathrm{~mol} \%$ 1$\mathbf{B H}_{3}$ in THF- $d_{8}(297 \mathrm{~K})$.

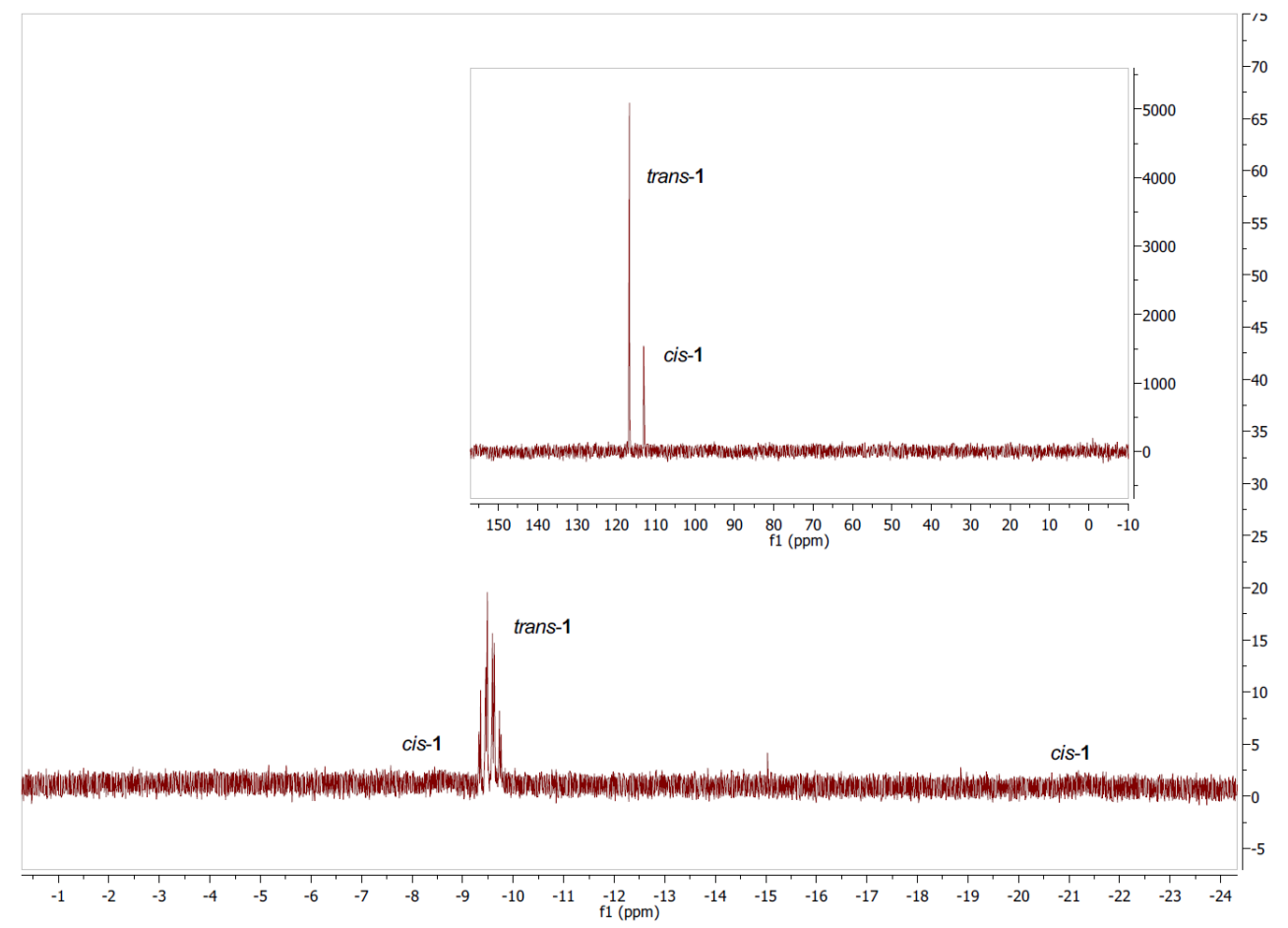

Figure S19. ${ }^{1} \mathrm{H}(300 \mathrm{MHz})$ and ${ }^{31} \mathrm{P}$ NMR (inset, $121 \mathrm{MHz}$ ) spectra of a reaction of HB with $100 \mathrm{~mol} \% \mathbf{2}$ in THF- $d_{8}(297 \mathrm{~K})$. 


\section{GC analysis}
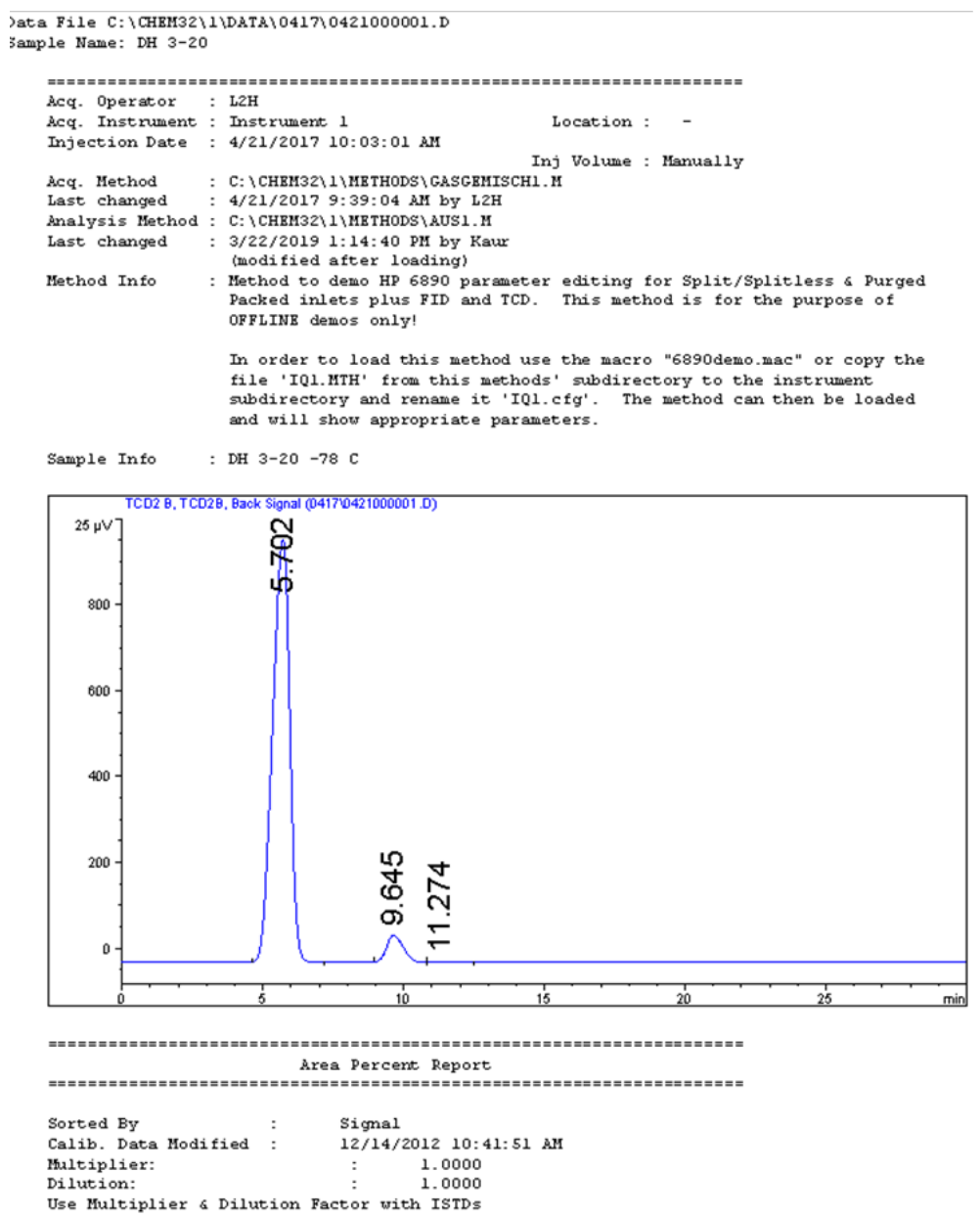

Use Multiplier \& Dilution Factor with ISTDs

Signal 1: TCD2 B, TCD2B, Back Signal

\begin{tabular}{|c|c|c|c|c|c|}
\hline $\begin{array}{c}\text { Pealk } \\
\#\end{array}$ & $\underset{[\text { min] }}{\operatorname{Ret} T \text { inme }}$ Type & $\begin{array}{l}\text { Width } \\
\text { [min] }\end{array}$ & $\begin{array}{c}\text { Area } \\
{\left[25 \mu V^{*} s\right]}\end{array}$ & Area & Name \\
\hline 1 & $1.000^{\circ}$ & 0.0000 & 0.00000 & 0.00000 & $1 \% \mathrm{H} 2$ \\
\hline 2 & $5.702 \mathrm{BB}$ & 0.6893 & $4.18734 \mathrm{e} 4$ & 94.38459 & \\
\hline 3 & $9.645 \mathrm{BV}$ & 0.6574 & 2468.64600 & 5. 56444 & ? \\
\hline 4 & 10.000 & 0.0000 & 0.00000 & 0.00000 & $10 \% \mathrm{H} 2$ \\
\hline 5 & $11.274 \mathrm{VB}$ & 0.6788 & 22.61292 & 0.05097 & \\
\hline 6 & 20.000 & 0.0000 & 0.00000 & 0.00000 & $204 \mathrm{H} 2$ \\
\hline 7 & 50.000 & 0.0000 & 0.00000 & 0.00000 & $50 \div \mathrm{H} 2$ \\
\hline 8 & 100.000 & 0.0000 & 0.00000 & 0.00000 & $100 \mathrm{H} 2$ \\
\hline \multicolumn{2}{|c|}{ Totals } & & $4.43647 \mathrm{e} 4$ & & \\
\hline
\end{tabular}

1 Warnings or Brrors :

Warning : Calibrated compound(s) not found

$* \pi *$ Bnd of Report $* * *$
$* \pi$

Figure S20. Gas chromatogram taken after catalytic dehydrogenation of HB with 2 mol\% 2 in THF at -78 ${ }^{\circ} \mathrm{C}$. The signal at $5.7 \mathrm{~min}$ corresponds to $\mathrm{H}_{2}$, the signal at 9.6 min corresponds to residual air $\left(\mathrm{O}_{2}, \mathrm{~N}_{2}\right)$ present in the syringe. 


\section{Computational Details}

\section{DFT computation of the catalytic mechanisms}

On the basis of our experimental findings we carried out B3PW91 density functional theory computation in the gas phase to investigate the reaction mechanism. The applicability of the B3PW91 functional was validated intensively and extensively. ${ }^{6}$ This included intensive and extensive testing, benchmarking of different methods with and without solvation effects and dispersion as well as intensive comparisons with the available experimental data and computational data for different transition metal PNP type complexes. ${ }^{6,7}$ On this basis, the good agreements between theory and experiment validate the B3PW91 gas phase calculations as reasonable.

Structure optimizations have been carried out at the B3PW9 ${ }^{8}$ density functional level of theory with the all-electron TZVP basis set ${ }^{9}$ using the Gaussian09 program package. ${ }^{10}$ The UltraFine integration grid was used for numerical integration. The optimized geometries are characterized as energy minimums at the potential energy surface from frequency calculations at the same level of theory, i.e., the energy minimum structure has only real frequencies, where an authentic transition state have only one imaginary vibration mode. It is noted that localization of the proposed transition states starting from HB-A and HB-B as well as HB-E for $\mathrm{H}_{2}$ formation was not possible despite employing different searching methods, such as a linear transit scan as well as a relaxed scan. For the dehydrogenation of $\mathbf{H B}$, using the amido complex 2 (starting Z-matrix, Table S2) for example, the calculation was started with B-H towards the Fe center (B$\mathrm{H}=1.234 \AA, \mathrm{Fe}-\mathrm{H}=1.955 \AA)$ and $\mathrm{N}-\mathrm{H}$ to the amido-N center $\left(\mathrm{N}_{1}-\mathrm{H}=1.090 \AA, \mathrm{N}_{2}-\mathrm{H}=2.120 \AA\right)$ with a $\mathrm{N}-\mathrm{B}-\mathrm{Fe}$ angle of $101.6^{\circ}$ by scanning the $\mathrm{Fe}-\mathrm{H}$ distance in increments of $-0.05 \AA$. After the first step with $\mathrm{Fe}-\mathrm{H}=1.905 \AA$ and $\mathrm{B}-\mathrm{H}=1.222 \AA$, the breaking $\mathrm{N}_{1}-\mathrm{H}$ becomes $1.080 \AA$ and the forming $\mathrm{N}_{2}-\mathrm{H}$ becomes $1.334 \AA$; the most important change affects the N-B-Fe angle from $101.6^{\circ}$ to $85.8^{\circ}$. The $\mathrm{N}_{2}-\mathrm{H}$ distance changes from $2.120 \AA$ to $1.334 \AA$ directly. The final state is the direct hydrogen transfer from the HB nitrogen atom to the amino nitrogen atom of the catalyst.

The Gibbs free energies, which are used for discussion and comparison are scaled with the thermal correction to Gibbs free energies at $298 \mathrm{~K}$. The computed energetic data and the optimized Cartesian coordinates (xyz) for all optimized structures are given in Table S1 and Table S2.

\section{B3PW91-SCRF-D3 calculations}

In addition to the gas phase results described in the previous section, we fully optimized all intermediates under consideration of solvation effects $\left[\mathrm{SCRF}=\left(\mathrm{SMD},{ }^{11}\right.\right.$ solvent $\left.\left.=\mathrm{THF}\right)\right]$ as well as dispersion correction using the latest GD3BJ version of Grimme's dispersion with Becke-Johnson damping ${ }^{12}$ and the B3PW91 functional for computing the thermal data scaled at $298 \mathrm{~K}$. The computed energetic data and the optimized Cartesian coordinates (xyz) for all optimized intermediates are listed in Table S1 and Table S3. The obtained Gibbs free energies are used for the comparison with those in gas phase in Figure 1.

Compared to the B3PW91 gas phase results, the B3PW91-SCRF-D3 calculations show that the hydrazine borane dehydrogenation $\left[\mathrm{N}_{2} \mathrm{H}_{4}-\mathrm{BH}_{3} \rightarrow \mathrm{H}_{2} \mathrm{~B}=\mathrm{NH}-\mathrm{NH}_{2}+\mathrm{H}_{2}\right]$ is only slightly exergonic by $1.02 \mathrm{kcal} / \mathrm{mol}$, a value which cannot explain the rapid formation of $\mathrm{H}_{2}$. In addition, amine catalyst dehydrogenation to amido catalyst $\left[\mathbf{1} \rightarrow \mathbf{2}+\mathrm{H}_{2}\right.$ ] is endergonic by $6.77 \mathrm{kcal} / \mathrm{mol}$; a value which supports the enhanced stability of amine complex (1) over the amido complex 2. However, this disagrees with the experimentally observed equilibrium between amine complex 1 and amido complex 2 under exchange of $\mathrm{H}_{2}$.

Most importantly, the reaction of amine complex 1 and hydrazine borane $\left[\mathbf{1}+\mathrm{N}_{2} \mathrm{H}_{4}-\mathrm{BH}_{3} \rightarrow \mathbf{2}+\right.$ $\mathrm{H}_{2} \mathrm{~B}=\mathrm{NH}-\mathrm{NH}_{2}+2 \mathrm{H}_{2} \mathrm{l}$ is endergonic by $5.75 \mathrm{kcal} / \mathrm{mol}$, indicating that this step is disfavored thermodynamically; $\boldsymbol{H B}-\boldsymbol{B}$ becomes a resting state on the potential energy surface by $5.28 \mathrm{kcal} / \mathrm{mol}$. Both findings do not agree with the experimental observation. Despite the endergonic energy difference between HB-B and the product $(11.03 \mathrm{kcal} / \mathrm{mol})$, we also to tried to locate the transition state for the second step of $\mathrm{H}_{2}$ formation from N-H and B-H starting from HB-B. However, no such transition state can be located and all attempts went back to HB-B. This reveals that another mechanism, such as the proposed acid and base mechanism in the main text, is indeed possible. 
For the reaction of amido complex 2 and hydrazine borane [ $2+\mathrm{N}_{2} \mathrm{H}_{4}-\mathrm{BH}_{3} \rightarrow \mathbf{1}+\mathrm{H}_{2} \mathrm{~B}=\mathrm{NH}-\mathrm{NH}_{2}$ ], the reaction is exergonic by $7.79 \mathrm{kcal} / \mathrm{mol}$; intermediate $\mathbf{H B}-\mathbf{E}$ becomes a resting state by $9.69 \mathrm{kcal} / \mathrm{mol}$, slightly more stable than the final state $\left[\mathbf{1}+\mathrm{H}_{2} \mathrm{~B}=\mathrm{NH}-\mathrm{NH}_{2}\right]$ by $1.90 \mathrm{kcal} / \mathrm{mol}$. In addition, we tried to locate the transition state of the B-H breaking starting from $\mathbf{H B}-\mathbf{E}$ for the hydride transfer from $\mathrm{B}-\mathrm{H}$ to $\mathrm{Fe}-$ $\mathrm{H}$, however, no such transition state could be located.

\section{Consideration of previous data}

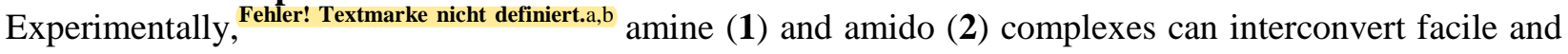
reversibly as well as form an equilibrium in benzene at room temperature. This reveals a rather low barrier and neutral reaction Gibbs free energy, which can be reproduced by B3PW91 gas phase calculation. ${ }^{13}$ The computed free energy barrier is $19.2 \mathrm{kcal} / \mathrm{mol}$ and the reaction is slightly exergonic by $0.6 \mathrm{kcal} / \mathrm{mol}$.

In contrast, including solvation and dispersion (B3PW91-SCRF-D3) raises the free energy barrier (25.5 $\mathrm{kcal} / \mathrm{mol})$ and makes the reaction much more endergonic $(9.1 \mathrm{kcal} / \mathrm{mol})$. The same results are reported when using M06-SCRF, ${ }^{14}$ which gives free energy barriers of 25.0 and $24.0 \mathrm{kcal} / \mathrm{mol}$ and endergonic reaction energies of 14.9 and $9.1 \mathrm{kcal} / \mathrm{mol}$ in THF and ethanol solution, respectively. These results do not agree with the experimentally observed facile and reversible exchange reaction under ambient conditions. Even worse results are found including solvation and dispersion for the transfer between 1-phenyl ethanol and acetone. ${ }^{15}$ Experimentally, this reaction requires a pressure tube to suppress $\mathrm{H}_{2}$ release and to shift the reaction to the product, whereas without the pressure tube only dehydrogenation of 1-phenyl ethanol was observed. This reveals that the barrier of $\mathrm{H}_{2}$ release should be lower than that of 1-phenyl ethanol dehydrogenation and acetone hydrogenation, which was confirmed by B3PW91 gas phase calculation (19.2 vs. 24.1 and $21.5 \mathrm{kcal} / \mathrm{mol}$, respectively). In contrast, opposite results are obtained when including solvation and dispersion, leading to much lower computed barriers of 1-phenyl ethanol dehydrogenation $(4.0 \mathrm{kcal} / \mathrm{mol})$ and acetone hydrogenation $(15.2 \mathrm{kcal} / \mathrm{mol})$ than the barrier $(25.5 \mathrm{kcal} / \mathrm{mol})$ of $\mathrm{H}_{2}$ release from amine (1) to amido (2) complexes. This suggests that this reaction can take place under ambient conditions, contradicting the experimental conditions of high temperature and high pressure. We note that similar observations have recently been found for Ru- and Mn-PNP systems involved in hydrogenation reactions, ${ }^{16}$ where the inclusion of solvation and dispersion also gave unrealistic barriers and reaction energies.

\section{Proposed catalytic mechanism for HB dehydrogenation (Figure S21)}

From the reaction of complex $\mathbf{1}$ and $\mathbf{H B}$, species HB-A turned out to be the most stable intermediate. This complex possesses only one hydrogen bond between $\mathrm{Fe}-\mathrm{H}$ and the proton of the central $\mathrm{NH}_{2}$ group $(\mathrm{NH}-$ $\mathrm{HFe}$ distance of $1.505 \AA$, H bonding is slightly exergonic by $0.20 \mathrm{kcal} / \mathrm{mol}$ ).

Starting from intermediate HB-A, despite numerous attempts, using different methods such as linear and transit scan as well as relaxed scan procedures, the proposed transition state (HB-1) for the formation of two equivalents of $\mathrm{H}_{2}$ to get complex 2 and $\mathrm{H}_{2} \mathrm{~N}-\mathrm{NH}=\mathrm{BH}_{2}$ could not be located. Furthermore, we evaluated the elimination of one $\mathrm{H}_{2}$ molecule between the hydrogen bonding via the frustrated Lewis Pair concept, starting from HB-A, i.e., one $\mathrm{H}_{2}$ molecule is situated in between the Fe center and the $\mathrm{N}$ center. Here, the structure optimization led to the direct formation of a structure with coordinated $\mathrm{H}_{2}$, while the proposed transition state (HB-2) could not be located.

Subsequent removal of $\mathrm{H}_{2}$ leads to the formation of intermediate $\mathbf{H B}-\mathbf{B}$, which is exergonic by $3.59 \mathrm{kcal} / \mathrm{mol}$. Starting from intermediate HB-B, noting that the HB-B-trans isomer is less stable by $3.99 \mathrm{kcal} / \mathrm{mol}$, it is also not possible to obtain the proposed transition state $\mathbf{H B}-\mathbf{3}$ for the second $\mathrm{H}_{2}$ formation event using relaxed scan method.

From intermediate HB-A to 2 and $\mathrm{H}_{2} \mathrm{~N}-\mathrm{NH}=\mathrm{BH}_{2}$, the reaction is exergonic by $11.61 \mathrm{kcal} / \mathrm{mol}$. However, the formation of complex $\mathbf{H B}-\mathbf{C}$ from 2 and $\mathrm{H}_{2} \mathrm{~N}-\mathrm{NH}=\mathrm{BH}_{2}$ is exergonic by $8.09 \mathrm{kcal} / \mathrm{mol}$, which is much smaller than the free energy $(-180.02 \mathrm{kcal} / \mathrm{mol})$ of the trimerization of $\mathrm{H}_{2} \mathrm{~N}-\mathrm{NH}=\mathrm{BH}_{2}$ to borazine and release of $\mathrm{NH}_{3}$. For the amine complex 1 route, hydrazine borane is dehydrogenated to $\mathrm{H}_{2} \mathrm{~N}-\mathrm{NH}=\mathrm{BH}_{2}$ and $\mathrm{H}_{2}$, and simultaneously amido complex $\mathbf{2}$ is formed.

For the dehydrogenation pathway of the amido complex 2 (Figure S21b), we first evaluated the possibility for a double hydrogen-bonding intermediate HB-D; however, structure optimizations led to the formation 
of intermediate HB-E. Here, the central N-H bond is already broken and the hydrogen atom is transferred to the amido nitrogen atom. The single hydrogen bonding interaction between B-H-Fe for this step is exergonic by $7.06 \mathrm{kcal} / \mathrm{mol}$. This step is exergonic by $7.06 \mathrm{kcal} / \mathrm{mol}$. In contrast, the formation of the hydrogen-bonding complex $(\mathbf{H B}-\mathbf{F})$ between the amido nitrogen atom and the central $\mathrm{NH}_{2}$ group of $\mathbf{H B}$ is endergonic by $3.46 \mathrm{kcal} / \mathrm{mol}$, and therefore, not competitive with HB-E. Starting from HB-E, we searched for the transition state of the hydride transfer from B to Fe (HB-4), which, despite using different approaches, was not successful. Instead, transition state optimization led directly to catalyst $\mathbf{1}$ and $\mathrm{H}_{2} \mathrm{~N}$ $\mathrm{NH}=\mathrm{BH}_{2}$. For the reaction of amido complex 2 as catalyst, $\mathbf{H B}$ is dehydrogenated to $\mathrm{H}_{2} \mathrm{~N}-\mathrm{NH}=\mathrm{BH}_{2}$, and at the same time amine complex $\mathbf{1}$ is regenerated.

(a) Amine complex 1 route, B3PW91/TZVP [B3PW91-SCRF-D3]
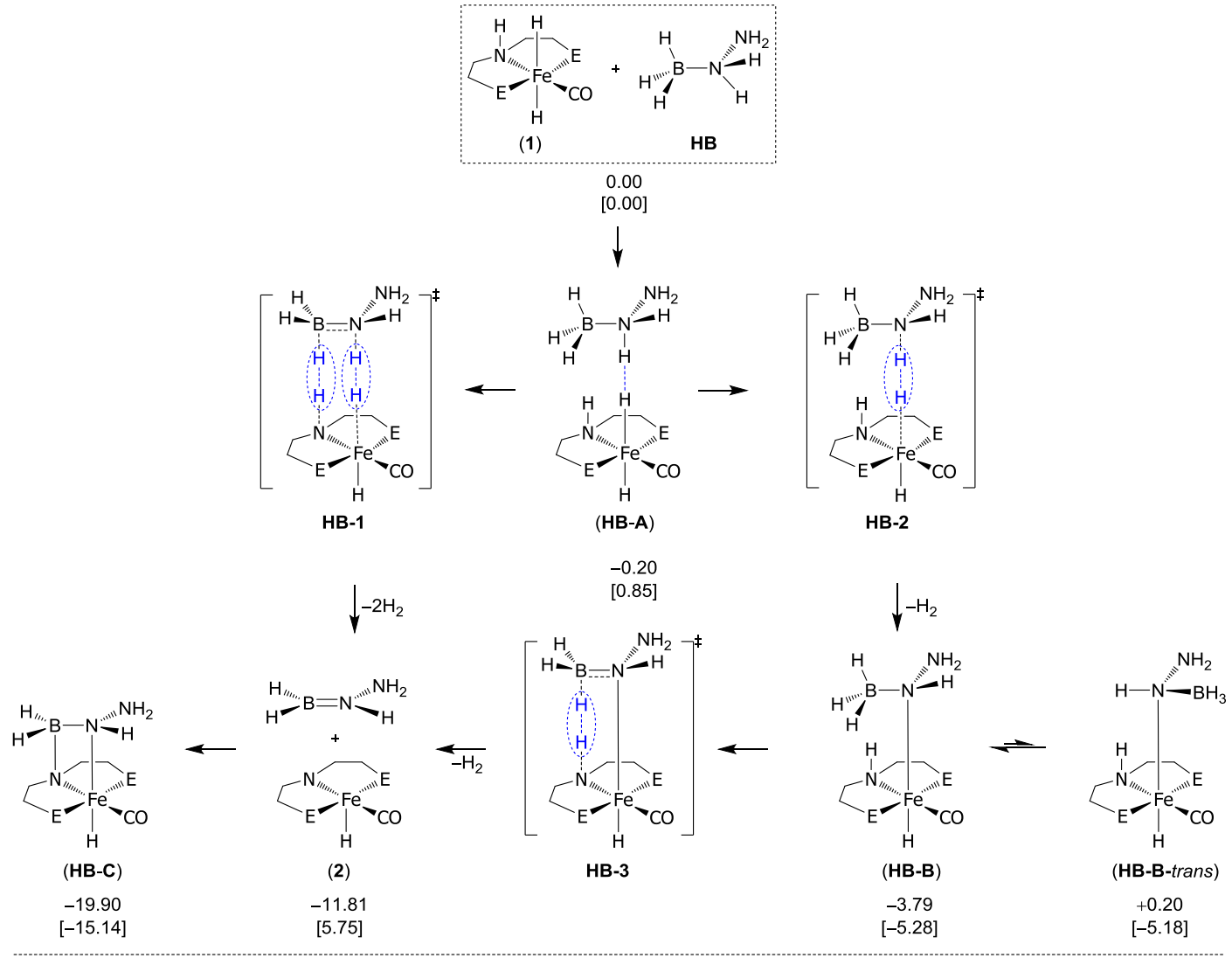

(b) Amido complex 2 route, B3PW91/TZVP [B3PW91-SCRF-D3]

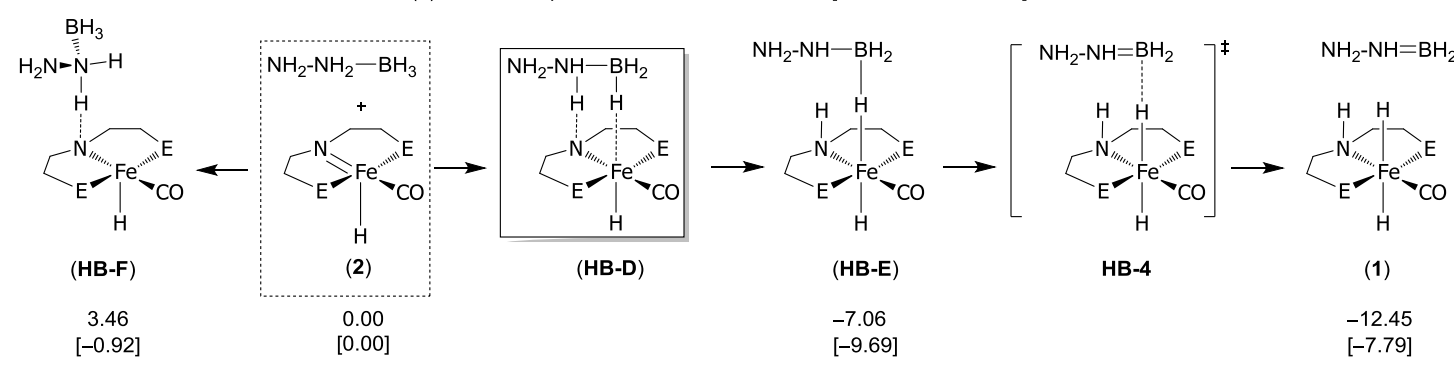

Figure S21. Reaction thermodynamics for the initial dehydrogenation of $\mathbf{H B}$ via amine complex $\mathbf{1}$ route (a, top) and amido complex 2 route (b, bottom), including the B3PW91 calculated Gibbs Free Energies relative to the starting material in $\mathrm{kcal} / \mathrm{mol}$ in gas phase. Values obtained from B3PW91-SCRF-D3 are given in brackets. The labels HB-1, HB-2, HB-3, and HB-4 represent proposed intermediate structures that could not be located as transition states. Further details can be found in Tables S1, S2, and S3. 


\section{Proposed catalytic mechanism for $\mathrm{AB}$ dehydrogenation}

Due to the similarity between $\mathbf{H B}$ and $\mathbf{A B}$ in structure and activity, we also computed the corresponding reaction for $\mathbf{A B}$ dehydrogenation by using catalysts $\mathbf{1}$ and $\mathbf{2}$. It is noted that Schneider and co-workers, ${ }^{17}$ carried out both the experimental and computational studies for this reaction. Starting from complex 2 and $\mathbf{A B}$, they observed that this reaction is active at room temperature and does not need additional heating, base and irradiation for activation as well as does not show an induction period. The reaction has first order rate dependence in catalyst and AB. Indeed, this is the same as found for the reaction of $\mathbf{2}$ with $\mathbf{H B}$. Along with their kinetic study, they also carried out B3LYP DFT computations on the basis of the truncated catalysts, i.e.; replacing the isopropyl substituents of catalysts $\mathbf{1}$ and $\mathbf{2}$ by methyl groups.

For comparison between $\mathbf{H B}$ and $\mathbf{A B}$, we computed the reactions of catalysts $\mathbf{1}$ and $\mathbf{2}$ with $\mathbf{A B}$ and the results are summarized in Figure S18.
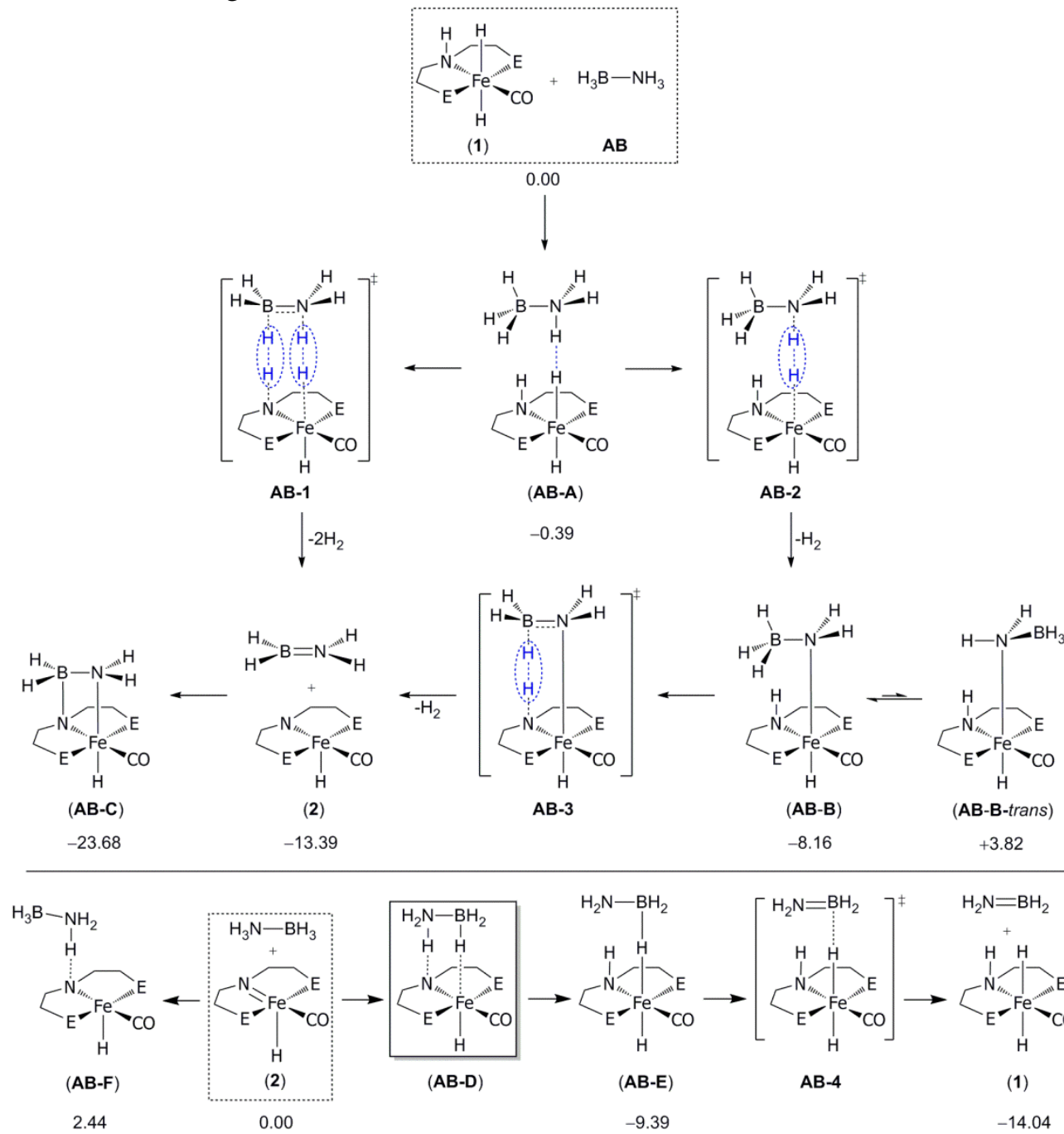

$-8.16$

$+3.82$

Figure S22. B3PW91 reaction thermodynamics for the initial dehydrogenation of AB via amine complex 1 route (a, top) and amido complex 2 route (b, bottom), including the calculated Gibbs Free Energies relative to the starting material in $\mathrm{kcal} / \mathrm{mol}$ in gas phase.

As expected, the reaction shows the behaviour with no substantial differences between $\mathbf{H B}$ and $\mathbf{A B}$. However, our computational results differ strongly from those reported before by Schneider and coworkers who employed truncated model complexes as catalysts, where the isopropyl groups were replaced by methyl groups, while we have used the real size complexes as catalysts. Moreover, a rather limited 
basis set (def2-SVP) was used previously for structure optimization and frequency calculation to obtain the thermal correction to Gibbs energies. In this work, we have used the all-electron TZVP basis set for optimization and frequency calculation to obtain thermal correction to Gibbs energies. Schneider and coworkers have used the B3LYP singlet-point energies on the basis of the B3LYP/def2-SVP optimized geometries, while we used the energies directly from structure optimization and frequency calculation at the same level of theory. Furthermore, our method used for the energy calculation is based on the fact that the two catalysts, the amine and amido forms are in a reversible equilibrium via $\mathrm{H}_{2}$ /under $\mathrm{H}_{2}$ atmosphere. Such equilibrium and reversibility reveal unambiguously that the reaction Gibbs free energy must be very small in both exergonic and endergonic directions. Our computed free energy barrier and reaction free energy from the amine to the amido complex is 17.4 and $0.3 \mathrm{kcal} / \mathrm{mol}$, respectively, in full agreement with the experimentally observed kinetics as well as equilibrium and reversibility. In contrast, Schneider's computed free energy barrier and reaction free energy from the amine to the amido complex is 24.7 and $6.4 \mathrm{kcal} / \mathrm{mol}$, respectively. Such a high barrier $(24.7 \mathrm{kcal} / \mathrm{mol})$ does not agree with the observed kinetics on one hand, and on the other hand, the highly endergonic reaction free energy $(6.4 \mathrm{kcal} / \mathrm{mol})$ does not show any equilibrium and reversibility. Also, these results are not in agreement with our experimental observations, i.e., a facile reaction at room temperature or in our case even at very low temperature ($78^{\circ} \mathrm{C}$ ). Most likely, the large differences between Schneider's work and our work is a result of the truncated catalytic system and the fact that their transition states (TS1, TS2 and TS6 in the work of Schneider and co-workers) either over or underestimate critical bond distances, resulting in energetic discussions that are based on unrealistic transition states. 
Table S1. Total electronic energies (HF, au), zero-point energies (ZPE, kcal/mol) as well as total energies including thermal enthalpy correction (Htot, au) and thermal free energy correction (Gtot, au); and the number of imaginary frequencies (NImag) at B3PW91 in gas phase as well as at B3PW91 in THF solution including dispersion correlation (B3PW91-SCRF-D3)

\begin{tabular}{|c|c|c|}
\hline B3PW91 & B3PW91 & B3PW91-SCRF-D3 \\
\hline H3B-NH3 & $\mathrm{N} 2 \mathrm{H} 4-\mathrm{BH} 3$ & $\mathrm{~N} 2 \mathrm{H} 4-\mathrm{BH} 3$ \\
\hline$H F=-83,2184724$ & $H F=-138.5333206$ & $H F=-138.5598234$ \\
\hline$Z P E=43.88206$ & $Z P E=55.14575$ & $Z P E=54.99988$ \\
\hline NImag $=0$ & NImag $=0$ & NImag $=0$ \\
\hline Htot=-83,143809 & Htot $=-138,439775$ & Htot $=-138.466498$ \\
\hline Gtot $=-83,170945$ & Gtot $=-138,471257$ & Gtot $=-138.498045$ \\
\hline BH3-NH3-TS & BH3-NH2-NH2-TS & \\
\hline$H F=-83,1590951$ & $H F=-138.4709753$ & \\
\hline$Z P E=39.76662$ & $Z P E=50.93690$ & \\
\hline NImag=1 (-1353.6697) & NImag= $1(-1303.1319)$ & \\
\hline Htot=-83,091141 & Htot $=-138,384208$ & \\
\hline Gtot $=-83,118992$ & Gtot $=-138,415533$ & \\
\hline $\mathrm{H} 2 \mathrm{~B}=\mathrm{NH} 2$ & $\mathrm{H} 2 \mathrm{~B}=\mathrm{NH}-\mathrm{NH} 2$ & $\mathrm{H} 2 \mathrm{~B}=\mathrm{NH}-\mathrm{NH} 2$ \\
\hline$H F=-82,0389631$ & $H F=-137.3500808$ & $H F=-137.3601661$ \\
\hline$Z P E=30.10359$ & $Z P E=41.01400$ & $Z P E=40.71885$ \\
\hline NImag $=0$ & NImag $=0$ & NImag $=0$ \\
\hline Htot $=-81,986820$ & Htot $=-137,279332$ & Htot $=-137.290659$ \\
\hline Gtot $=-82,012735$ & Gtot $=-137,310524$ & Gtot $=-137.319906$ \\
\hline $\mathrm{H} 2$ & $\mathrm{~B} 2 \mathrm{H} 6$ & $\mathrm{H} 2$ \\
\hline$E_{\text {tot }}=-1,1786359$ & $H F=-53.2804265$ & $H F=-1.1782934$ \\
\hline $\mathrm{ZPE}=6.31541$ & $Z P E=39.35171$ & $Z P E=6.29142$ \\
\hline NImag $=0$ & NImag $=0$ & NImag $=0$ \\
\hline$H_{\text {tot }}=-1,165267$ & Htot $=-53.213155$ & Htot $=-1.164963$ \\
\hline$G_{\text {tot }}=-1,180065$ & Gtot $=-53,239545$ & Gtot $=-1.179763$ \\
\hline B3N3H6 (borazine) & & B3N3H6 (borazine) \\
\hline$H F=-242.6489822$ & & $H F=-242.6675256$ \\
\hline$Z P E=58.53091$ & & $Z P E=58.04541$ \\
\hline NImag $=0$ & & NImag $=0$ \\
\hline Htot=-242,549522 & & Htot $=-242.568788$ \\
\hline Gtot $=-242,582817$ & & Gtot $=-242.602159$ \\
\hline $\mathrm{H}_{3} \mathrm{~B}_{-}$ & $\mathrm{H}$ & $\mathrm{H} H$ \\
\hline $\mathrm{HF}=-2774.3191697$ & $E_{\text {tot }}=-2747,6560051$ & $H F=-2747.8419491$ \\
\hline$Z P E=358.14728$ & $\mathrm{ZPE}=337.84605$ & $Z P E=338.18801$ \\
\hline NImag $=0$ & NImag $=0$ & NImag $=0$ \\
\hline Htot=-2773.715631 & $\mathrm{H}_{\mathrm{tot}}=-2747,086713$ & Htot $=-2747.272325$ \\
\hline Gtot $=-2773,807353$ & $G_{\text {tot }}=-2747,175333$ & Gtot $=-2747.361053$ \\
\hline
\end{tabular}




\begin{tabular}{|c|c|c|}
\hline $\begin{array}{l}\mathrm{E}_{\mathrm{tot}}=-2747,6216128 \\
\mathrm{ZPE}=334.05204 \\
\mathrm{NImag}=1(-976 \mathrm{~cm}-1) \\
\mathrm{H}_{\mathrm{tot}}=-2747,058396 \\
\mathrm{G}_{\mathrm{tot}}=-2747,147494\end{array}$ & $\begin{array}{l}E_{\text {tot }}=-2746.454005 \\
Z P E=324.91590 \\
N I m a g=0 \\
H_{\text {tot }}=-2745,905471 \\
G_{\text {tot }}=-2745,994751\end{array}$ & $\begin{array}{l}\text { (iP) } \\
\mathrm{HF}=-2746.6290962 \\
\mathrm{ZPE}=324.67597 \\
\mathrm{NImag}=0 \\
\text { Htot }=-2746.081033 \\
\text { Gtot }=-2746.170503\end{array}$ \\
\hline $\begin{array}{l}\mathrm{ZPE}=382.85880 \\
\mathrm{NImag}=0 \\
\mathrm{H} \text { tot }=-2830,246769\end{array}$ & $\begin{array}{l}\mathrm{HF}=-2886.206704 \\
\mathrm{ZPE}=393.37364 \\
\mathrm{~N} \text { tmag=0 } \\
\text { Gtot=-2885.542352 }\end{array}$ & $\begin{array}{l}\mathrm{HF}=-2886.4213229 \\
\mathrm{ZPE}=394.32534 \\
\mathrm{NImag}=0 \\
\text { Gtot=-2885.756267 }\end{array}$ \\
\hline $\begin{array}{l}\mathrm{HF}=-2829.7099267 \\
\mathrm{HIm}=371.48813 \\
\text { Htot }=-2829,083598 \\
\text { Gtot }=-2829,179222\end{array}$ & $\begin{array}{l}\mathrm{HF}=-2885.0199093 \\
\mathrm{ZPE}=382.39824 \\
\mathrm{H} \text { tot }=-2884.375156\end{array}$ & $\begin{array}{l}\mathrm{HF}=-2885.2369277 \\
\mathrm{ZPE}=382.89921 \\
\mathrm{NImag}=0 \\
\mathrm{Htot}=-2884.591866\end{array}$ \\
\hline $\begin{array}{l}\text { HF=-2829.6901229 } \\
\mathrm{ZPE}=370.72264 \\
\mathrm{NImag}=0 \\
\text { Htot=-2829.064786 } \\
\text { Gtot=-2829,160127 }\end{array}$ & $\begin{array}{l}\mathrm{HF}=-2885.0136473 \\
\mathrm{ZPE}=382.00609 \\
\mathrm{NImag}=0 \\
\mathrm{Htot}=-2884.369449 \\
\text { Gtot }=-2884,466199\end{array}$ & $\begin{array}{l}\mathrm{HF}=-2885.2383809 \\
\mathrm{ZPE}=383.06198 \\
\mathrm{NImag}=0 \\
\mathrm{Htot}=-2884.593267 \\
\text { Gtot }=-2884.687591\end{array}$ \\
\hline
\end{tabular}




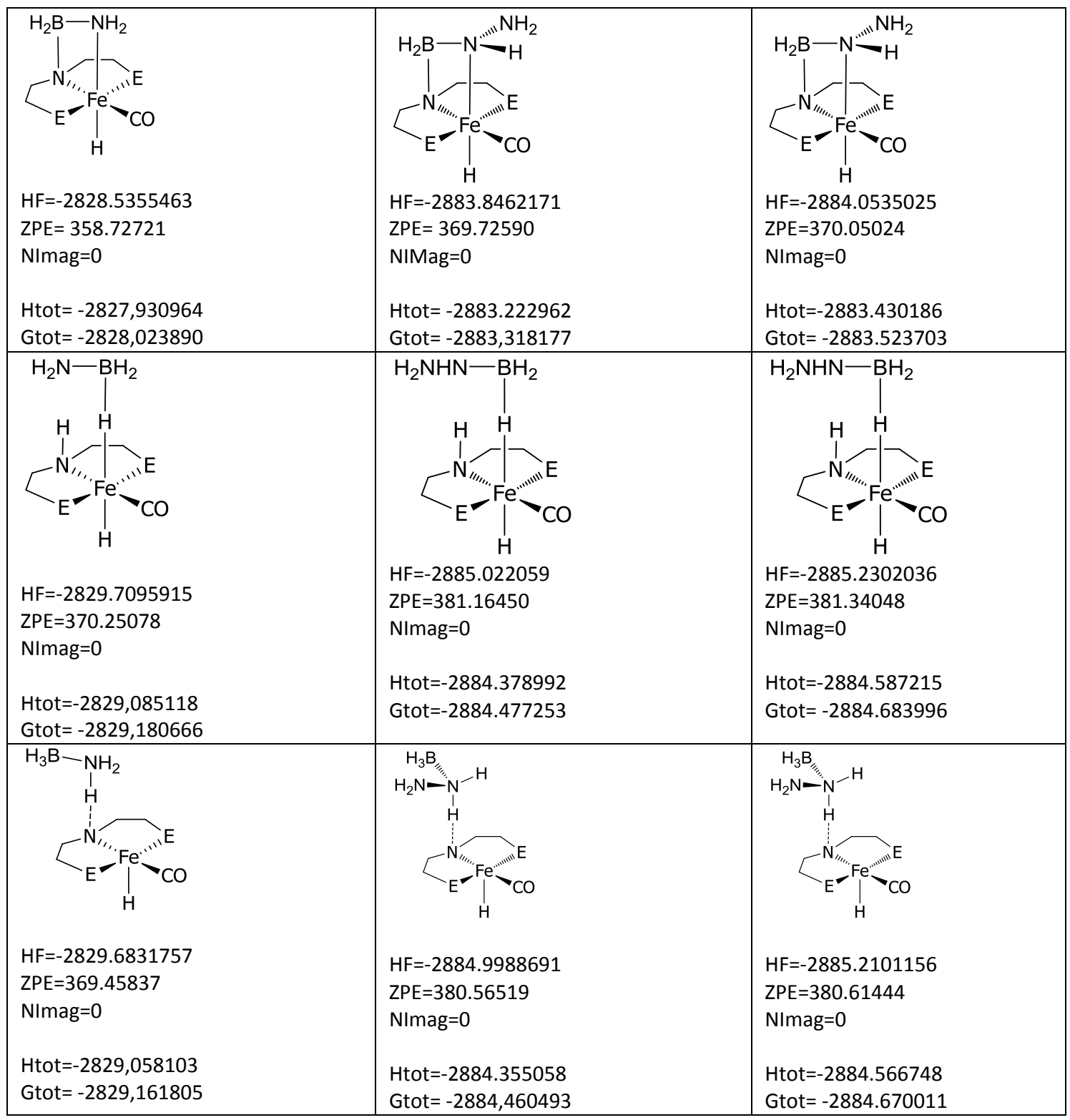

Table S2. B3PW91 optimized Cartesian coordinates in gas phase

\begin{tabular}{|c|c|}
\hline H3B-NH3 & $\mathrm{N} 2 \mathrm{H} 4-\mathrm{BH} 3$ \\
\hline B,0,-0.0000000461,0.0000000672,1.1674975971 & $\mathrm{H}, 0,-0.0986554843,-0.1158083297,0.1483673596$ \\
\hline$H, 0,1.1696154625,0.0000001318,1.4840530044$ & $\mathrm{~B}, 0,0.1668676165,-0.2224081213,1.3274488202$ \\
\hline$H, 0,-0.5848077445,-1.0129167082,1.4840530044$ & $\mathrm{H}, 0,0.0759355735,-1.3615868262,1.7388101076$ \\
\hline$H, 0,-0.5848078563,1.012916778,1.4840530044$ & $\mathrm{H}, 0,-0.4078219298,0.5897086042,2.0241648432$ \\
\hline $\mathrm{N}, 0,-0.0000000461,0.0000000672,-0.483586481$ & $\mathrm{~N}, 0,1.7329433388,0.1475845079,1.4523318181$ \\
\hline$H, 0,0.4739046279,-0.8208268014,-0.8486901863$ & $H, 0,1.9151995656,1.0849293396,1.1009612978$ \\
\hline $\mathrm{H}, 0,0.4739045372,0.8208269881,-0.8486901863$ & $H, 0,2.300188085,-0.467969238,0.873867565$ \\
\hline \multirow{3}{*}{$H, 0,-0.9478093034,0.0000000148,-0.8486901863$} & $N, 0,2.3108108968,0.0960017069,2.7847121975$ \\
\hline & $\mathrm{H}, 0,1.7056495186,0.6984604182,3.3398789371$ \\
\hline & $\mathrm{H}, 0,2.0873861097,-0.8413211063,3.1147034239$ \\
\hline BH3-NH3-TS & BH3-NH2-NH2-TS \\
\hline $\mathrm{N}, 0,-0.1371721471,0.0007081811,0.8049513639$ & B,0,0.0076279982,0.1278723538,-0.024998074 \\
\hline$H, 0,-0.4873710768,0.839825536,1.2370354584$ & $\mathrm{~N}, 0,1.5630016215,0.1034699256,0.1751643244$ \\
\hline $\mathrm{H}, 0,-0.495954749,-0.8351194339,1.236337583$ & $\mathrm{H}, 0,-0.3942650422,1.1456113701,-0.5102453281$ \\
\hline$H, 0,1.0756568894,-0.0052206392,0.1381301969$ & $\mathrm{H}, 0,-0.6219170548,-0.4133891555,0.8413722536$ \\
\hline
\end{tabular}




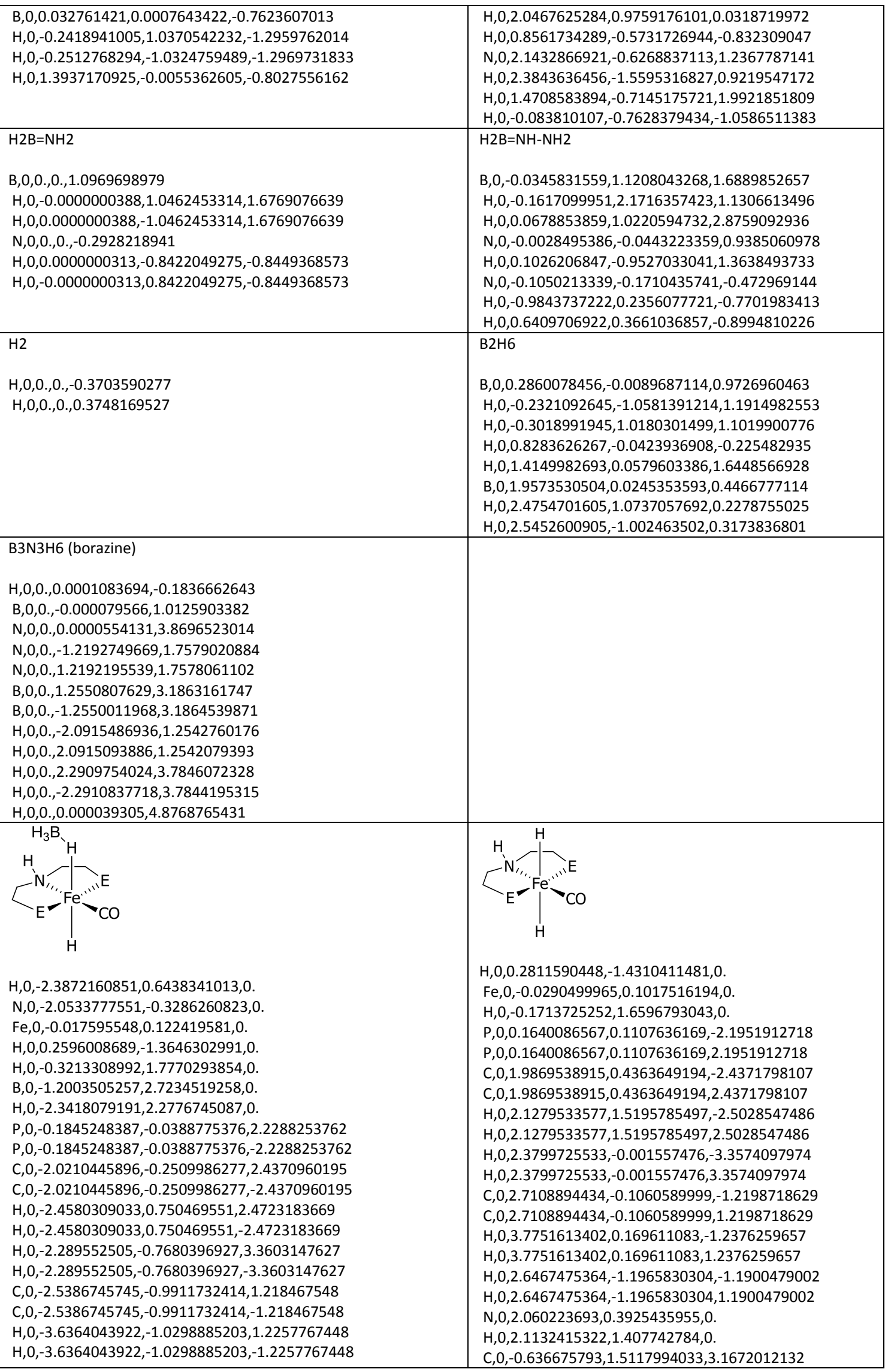


H,0,-2.1737665104,-2.0222151851,1.2035786042 $\mathrm{H}, 0,-2.1737665104,-2.0222151851,-1.2035786042$ C,0,0.215420999,1.4616412214,-3.2811608241 C,0,0.215420999,1.4616412214,3.2811608241 $\mathrm{H}, 0,-0.1418258877,2.2541763227,-2.6131800902$ $\mathrm{H}, 0,-0.1418258877,2.2541763227,2.6131800902$ C,0,0.499575943,-1.6060719632,-3.013148341 C,0,0.499575943,-1.6060719632,3.013148341 $\mathrm{H}, 0,-0.1139273425,-2.3667535349,-2.5141005291$ $\mathrm{H}, 0,-0.1139273425,-2.3667535349,2.5141005291$ C, $0,1.689428582,0.417078499,0$.

$0,0,2.8336037859,0.580392323,0$.

C, $0,0.2989714565,-1.7416140444,4.5205480179$ C,0,0.2989714565,-1.7416140444,-4.5205480179 $\mathrm{H}, 0,-0.7270472343,-1.5410898979,4.8357109$ $\mathrm{H}, 0,-0.7270472343,-1.5410898979,-4.8357109$ $H, 0,0.9586955354,-1.0695290435,5.072633751$ $H, 0,0.9586955354,-1.0695290435,-5.072633751$ $H, 0,0.5428002992,-2.761568412,4.8335869801$ $\mathrm{H}, 0,0.5428002992,-2.761568412,-4.8335869801$ C,0,1.9508377295,-1.8740137037,2.6216183141 C,0,1.9508377295,-1.8740137037,-2.6216183141 $\mathrm{H}, 0,2.631643568,-1.1326560461,3.0442255561$ $H, 0,2.631643568,-1.1326560461,-3.0442255561$ $H, 0,2.0732227288,-1.8721497683,1.5390386917$ $\mathrm{H}, 0,2.0732227288,-1.8721497683,-1.5390386917$ $\mathrm{H}, 0,2.2594134421,-2.854545332,2.9968660671$ $H, 0,2.2594134421,-2.854545332,-2.9968660671$ C,0,1.7194127762,1.6552730622,-3.4673757271 C,0,1.7194127762,1.6552730622,3.4673757271 $H, 0,2.1410541583,0.9214874669,-4.1591526819$ $\mathrm{H}, 0,2.1410541583,0.9214874669,4.1591526819$ $H, 0,2.2664702068,1.5915598192,-2.5260315971$ $H, 0,2.2664702068,1.5915598192,2.5260315971$ $H, 0,1.910082365,2.6457935569,-3.8901264604$ $\mathrm{H}, 0,1.910082365,2.6457935569,3.8901264604$ C,0,-0.5306220021,1.5911671828,-4.6079858922 C, $0,-0.5306220021,1.5911671828,4.6079858922$ $\mathrm{H}, 0,-1.6100082686,1.4754812095,-4.4966829596$ $\mathrm{H}, 0,-1.6100082686,1.4754812095,4.4966829596$ $\mathrm{H}, 0,-0.1868365049,0.8711627599,-5.3518435152$ $\mathrm{H}, 0,-0.1868365049,0.8711627599,5.3518435152$ $\mathrm{H}, 0,-0.3572022442,2.5908731864,-5.0182702269$ $\mathrm{H}, 0,-0.3572022442,2.5908731864,5.0182702269$ $\mathrm{H}, 0,-0.9711155498,3.353392363,1.0105554823$ $H, 0,-0.9711155498,3.353392363,-1.0105554823$

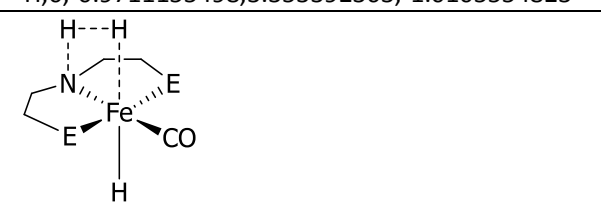

$\mathrm{H}, 0,-0.0998684559,-1.2540203405,0$. $\mathrm{Fe}, 0,0.0027898429,0.2597129748,0$. $P, 0,0.2125537439,0.1256941302,-2.2183969637$ $P, 0,0.2125537439,0.1256941302,2.2183969637$ C,0,2.0443101894,0.3304006545,-2.4289268738 C,0,2.0443101894,0.3304006545,2.4289268738 $\mathrm{H}, 0,2.2473392447,1.4042733987,-2.4687124893$ $\mathrm{H}, 0,2.2473392447,1.4042733987,2.4687124893$ $\mathrm{H}, 0,2.4208654912,-0.1202665918,-3.3494695887$ $\mathrm{H}, 0,2.4208654912,-0.1202665918,3.3494695887$ C,0,2.6962851599,-0.2641896681,-1.192527624 C,0,2.6962851599,-0.2641896681,1.192527624 $\mathrm{H}, 0,3.7756644219,-0.036377561,-1.2058770674$ $\mathrm{H}, 0,3.7756644219,-0.036377561,1.2058770674$
C,0,-0.636675793,1.5117994033,-3.1672012132 $\mathrm{H}, 0,-0.4513221632,2.3373550998,2.4705604147$ $\mathrm{H}, 0,-0.4513221632,2.3373550998,-2.4705604147$ C,0,-0.0683573389,-1.5199093147,3.1128034036 C, $0,-0.0683573389,-1.5199093147,-3.1128034036$ $\mathrm{H}, 0,0.7462399127,-2.1070983089,2.6704004994$ $\mathrm{H}, 0,0.7462399127,-2.1070983089,-2.6704004994$ C,0,-1.7360299226,-0.0995304003,0.

$0,0,-2.8912965902,-0.2238745118,0$. C,0,0.1219414128,-1.4956474772,-4.626584331 C,0,0.1219414128,-1.4956474772,4.626584331 $H, 0,1.0312795504,-0.9746297651,-4.934257268$ $\mathrm{H}, 0,1.0312795504,-0.9746297651,4.934257268$ $\mathrm{H}, 0,-0.7245307955,-1.0216745846,-5.1278646794$ $\mathrm{H}, 0,-0.7245307955,-1.0216745846,5.1278646794$ $\mathrm{H}, 0,0.1871184338,-2.5200077056,-5.0069794668$ $\mathrm{H}, 0,0.1871184338,-2.5200077056,5.0069794668$ C, $0,-1.3726201135,-2.2135074412,-2.7261287023$ C,0,-1.3726201135,-2.2135074412,2.7261287023 $\mathrm{H}, 0,-2.2484267013,-1.6694022759,-3.0864394107$ $\mathrm{H}, 0,-2.2484267013,-1.6694022759,3.0864394107$ $\mathrm{H}, 0,-1.4538684506,-2.3126409283,-1.6438862905$ $\mathrm{H}, 0,-1.4538684506,-2.3126409283,1.6438862905$ $H, 0,-1.4046720778,-3.2154225665,-3.1660439909$ $\mathrm{H}, 0,-1.4046720778,-3.2154225665,3.1660439909$ C,0,-2.150359315,1.3377061248,3.2754820937 C,0,-2.150359315,1.3377061248,-3.2754820937 $\mathrm{H}, 0,-2.4125040929,0.5334887832,3.9675268245$ $\mathrm{H}, 0,-2.4125040929,0.5334887832,-3.9675268245$ $\mathrm{H}, 0,-2.6088322943,1.1204885908,2.3105516414$ $\mathrm{H}, 0,-2.6088322943,1.1204885908,-2.3105516414$ $\mathrm{H}, 0,-2.6032786514,2.2563803917,3.6606474959$ $\mathrm{H}, 0,-2.6032786514,2.2563803917,-3.6606474959$ C,0,-0.0263993729,1.8811392102,4.5190418979 C,0,-0.0263993729,1.8811392102,-4.5190418979 $\mathrm{H}, 0,1.0576446137,2.0048077207,4.4751930626$ $\mathrm{H}, 0,1.0576446137,2.0048077207,-4.4751930626$ $\mathrm{H}, 0,-0.2481571977,1.1407624267,5.2882389782$ $\mathrm{H}, 0,-0.2481571977,1.1407624267,-5.2882389782$ $\mathrm{H}, 0,-0.4460507125,2.8342886424,4.8571037653$ $\mathrm{H}, 0,-0.4460507125,2.8342886424,-4.8571037653$

$\mathrm{H}, 0,-1.0283455224,-0.6733319678,0$.

$\mathrm{Fe}, 0,0.049666565,0.3534828681,0$.

$P, 0,0.2027598633,0.1498968879,-2.2078963135$ $P, 0,0.2027598633,0.1498968879,2.2078963135$ C,0,2.0249047616,0.3920817122,-2.4214005959 C,0,2.0249047616,0.3920817122,2.4214005959 $\mathrm{H}, 0,2.1871397402,1.4735926939,-2.4283925213$ $\mathrm{H}, 0,2.1871397402,1.4735926939,2.4283925213$ $\mathrm{H}, 0,2.4274253203,-0.0185294639,-3.3502251656$ $\mathrm{H}, 0,2.4274253203,-0.0185294639,3.3502251656$ C, $0,2.6887835686,-0.2075459074,-1.1919721458$ C, $0,2.6887835686,-0.2075459074,1.1919721458$ $\mathrm{H}, 0,3.6979533433,0.2188266246,-1.0792942741$ $\mathrm{H}, 0,3.6979533433,0.2188266246,1.0792942741$ 
$\mathrm{H}, 0,2.6147546965,-1.3685288057,-1.2326412196$ $\mathrm{H}, 0,2.6147546965,-1.3685288057,1.2326412196$ $\mathrm{N}, 0,2.0807100435,0.2568925267,0$.

C, $0,-0.5125062379,1.5025641956,3.2778849203$ C, $0,-0.5125062379,1.5025641956,-3.2778849203$ $\mathrm{H}, 0,-0.3401739041,2.3614286841,2.6184390047$ $\mathrm{H}, 0,-0.3401739041,2.3614286841,-2.6184390047$ C, $0,-0.1354481424,-1.5489561639,2.9936104578$ C, $0,-0.1354481424,-1.5489561639,-2.9936104578$ $\mathrm{H}, 0,0.6039437677,-2.1621602874,2.4632962946$ $\mathrm{H}, 0,0.6039437677,-2.1621602874,-2.4632962946$ C, $0,-1.7278628312,0.370352653,0$.

$0,0,-2.8858508492,0.406827069,0$.

C, $0,0.1259725755,-1.6700572494,-4.4929380851$ C, $0,0.1259725755,-1.6700572494,4.4929380851$ $\mathrm{H}, 0,1.1029602689,-1.2817571833,-4.7880117125$ $\mathrm{H}, 0,1.1029602689,-1.2817571833,4.7880117125$ $\mathrm{H}, 0,-0.6363464822,-1.1491720953,-5.0761039232$ $\mathrm{H}, 0,-0.6363464822,-1.1491720953,5.0761039232$ $\mathrm{H}, 0,0.0912050965,-2.7236929809,-4.7867741114$ $\mathrm{H}, 0,0.0912050965,-2.7236929809,4.7867741114$ C, $0,-1.5150950998,-2.0891157034,-2.6235312828$ C, $0,-1.5150950998,-2.0891157034,2.6235312828$ $\mathrm{H}, 0,-2.3175738322,-1.5263864257,-3.1047638854$ $\mathrm{H}, 0,-2.3175738322,-1.5263864257,3.1047638854$ $\mathrm{H}, 0,-1.6708750148,-2.0547347859,-1.5454275891$ $\mathrm{H}, 0,-1.6708750148,-2.0547347859,1.5454275891$ $\mathrm{H}, 0,-1.6038635724,-3.1301147394,-2.9492544827$ $\mathrm{H}, 0,-1.6038635724,-3.1301147394,2.9492544827$ C, $0,-2.0221746576,1.3677886036,3.4719543529$ C, $0,-2.0221746576,1.3677886036,-3.4719543529$ $\mathrm{H}, 0,-2.2644451304,0.5598686739,4.1666389708$ $\mathrm{H}, 0,-2.2644451304,0.5598686739,-4.1666389708$ $\mathrm{H}, 0,-2.5485487199,1.1805714855,2.5357674643$ $\mathrm{H}, 0,-2.5485487199,1.1805714855,-2.5357674643$ $\mathrm{H}, 0,-2.4235709529,2.2914613249,3.8994981581$ $\mathrm{H}, 0,-2.4235709529,2.2914613249,-3.8994981581$ C, $0,0.1882504442,1.7836137225,4.6068033351$ C, $0,0.1882504442,1.7836137225,-4.6068033351$ $\mathrm{H}, 0,1.2652740737,1.9160436473,4.4936690546$ $\mathrm{H}, 0,1.2652740737,1.9160436473,-4.4936690546$ $\mathrm{H}, 0,0.0213514829,0.988908089,5.3350732836$ $\mathrm{H}, 0,0.0213514829,0.988908089,-5.3350732836$ $\mathrm{H}, 0,-0.2094868688,2.7078554882,5.0382378409$ $\mathrm{H}, 0,-0.2094868688,2.7078554882,-5.0382378409$ $\mathrm{H}, 0,0.3494235179,1.8859772101,0$. $H, 0,1.1988722366,1.422224916,0$.

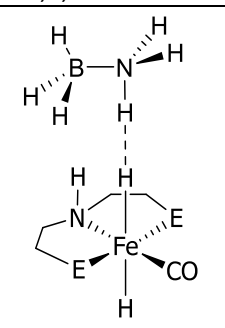

$\mathrm{H}, 0,-2.137249839,-1.1425545226,0.0005044336$ $\mathrm{N}, 0,-1.2119786118,-1.5748648974,0.0005365304$ $\mathrm{Fe}, 0,0.1555779329,0.0386271354,0.0000674979$ $\mathrm{H}, 0,-1.0517759409,1.0618761199,-0.0000021576$ $\mathrm{H}, 0,-2.5591464241,1.3784465829,-0.000441543$ $\mathrm{N}, 0,-3.5968166984,1.5145130995,-0.0006868458$ B, $0,-4.3631328657,0.0847563004,-0.0004530865$ $H, 0,1.2630993144,-1.0477128869,0.0001737363$
$\mathrm{H}, 0,2.8419441515,-1.2911493971,-1.3382209019$ $\mathrm{H}, 0,2.8419441515,-1.2911493971,1.3382209019$ $\mathrm{N}, 0,1.8949111581,0.0550858661,0$.

C, $0,-0.5721608947,1.4356130903,3.3386435716$ C, $0,-0.5721608947,1.4356130903,-3.3386435716$ $\mathrm{H}, 0,-0.4220005641,2.3414373553,2.7393706515$ $\mathrm{H}, 0,-0.4220005641,2.3414373553,-2.7393706515$ C, $0,-0.0901419093,-1.5832878624,2.8836581809$ C, $0,-0.0901419093,-1.5832878624,-2.8836581809$ $\mathrm{H}, 0,0.6322968029,-2.1482337767,2.2808451974$ $\mathrm{H}, 0,0.6322968029,-2.1482337767,-2.2808451974$ C, $0,-1.248551855,1.4951331108,0$.

$0,0,-2.0921153304,2.2943560454,0$. C, $0,0.2451178954,-1.802721665,-4.3576461069$ C, $0,0.2451178954,-1.802721665,4.3576461069$ $\mathrm{H}, 0,1.2233332451,-1.4077254473,-4.6376429926$ $\mathrm{H}, 0,1.2233332451,-1.4077254473,4.6376429926$ $\mathrm{H}, 0,-0.5037216105,-1.3465808815,-5.0087516527$ $\mathrm{H}, 0,-0.5037216105,-1.3465808815,5.0087516527$ $\mathrm{H}, 0,0.2507262112,-2.8749862907,-4.5777771907$ $\mathrm{H}, 0,0.2507262112,-2.8749862907,4.5777771907$ C, $0,-1.4776261357,-2.1302210637,-2.5527923378$ C, $0,-1.4776261357,-2.1302210637,2.5527923378$ $\mathrm{H}, 0,-2.2543936054,-1.6504284219,-3.1515595891$ $\mathrm{H}, 0,-2.2543936054,-1.6504284219,3.1515595891$ $\mathrm{H}, 0,-1.7197027658,-1.9813327552,-1.5003253428$ $\mathrm{H}, 0,-1.7197027658,-1.9813327552,1.5003253428$ $\mathrm{H}, 0,-1.5137867808,-3.2025019143,-2.7684629986$ $\mathrm{H}, 0,-1.5137867808,-3.2025019143,2.7684629986$ C, $0,-2.0786009898,1.2368337458,3.4987091768$ C, $0,-2.0786009898,1.2368337458,-3.4987091768$ $\mathrm{H}, 0,-2.3011411231,0.3925333791,4.1553864457$ $\mathrm{H}, 0,-2.3011411231,0.3925333791,-4.1553864457$ $\mathrm{H}, 0,-2.5771280936,1.0694837653,2.5430400991$ $\mathrm{H}, 0,-2.5771280936,1.0694837653,-2.5430400991$ $\mathrm{H}, 0,-2.5237287179,2.1272895385,3.9519599097$ $\mathrm{H}, 0,-2.5237287179,2.1272895385,-3.9519599097$ C, $0,0.1063263151,1.6540186199,4.6898225567$ C, $0,0.1063263151,1.6540186199,-4.6898225567$ $\mathrm{H}, 0,1.1779384325,1.8368258498,4.5942659903$ $\mathrm{H}, 0,1.1779384325,1.8368258498,-4.5942659903$ $\mathrm{H}, 0,-0.0320476058,0.8047443952,5.3601901343$ $\mathrm{H}, 0,-0.0320476058,0.8047443952,-5.3601901343$ $\mathrm{H}, 0,-0.3312385413,2.5300488273,5.1791471137$ $\mathrm{H}, 0,-0.3312385413,2.5300488273,-5.1791471137$

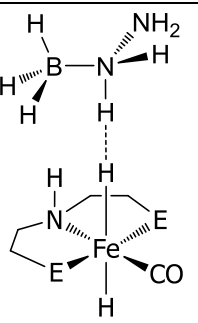

$\mathrm{H}, 0,-0.0606776945,1.6964256185,1.6391777347$ $\mathrm{N}, 0,0.0118216279,0.699926051,1.8507266949$ $\mathrm{Fe}, 0,0.0268279239,-0.2691724744,-0.0308734732$ $\mathrm{H}, 0,-0.0800937343,1.1334740493,-0.7666531202$ $\mathrm{H}, 0,0.1660357002,2.6123617037,-0.6278339829$ $\mathrm{N}, 0,0.1120086946,3.6601916694,-0.6105389165$ B,0,-0.1624912212,4.1259149337,0.9005470093 H, $, 0.0 .1095941946,-1.5890889309,0.7743577112$ $\mathrm{P}, 0,2.2344138735,-0.1738515547,0.1531990808$ 
$P, 0,0.1085566322,-0.175491169,2.2051290526$ $P, 0,0.1081235898,-0.1764362158,-2.2049156286$ C, $0,-1.1598983034,-1.5176859804,2.4438051899$ C, $0,-1.1603183556,-1.5187875302,-2.4427561125$ $\mathrm{H}, 0,-2.142730683,-1.044925747,2.5201293144$ $\mathrm{H}, 0,-2.1431832199,-1.0460963518,-2.5190887637$ $\mathrm{H}, 0,-0.9994131185,-2.1005462996,3.3533659084$ $\mathrm{H}, 0,-0.9999877518,-2.1020413169,-3.352090704$ C,0,-1.1192609269,-2.4034259466,1.2140347391 C, $0,-1.119434634,-2.4039789321,-1.2126005389$ $\mathrm{H}, 0,-1.9324500951,-3.1410549062,1.2403471623$ $\mathrm{H}, 0,-1.9326018932,-3.1416490459,-1.2384442006$ $\mathrm{H}, 0,-0.1721091319,-2.9470072428,1.1616651608$ $\mathrm{H}, 0,-0.1722520094,-2.9474946112,-1.1601340488$ C, $0,-0.5721475371,1.235741521,-3.2520904075$ C, $0,-0.571429691,1.2371925303,3.2518041758$ $\mathrm{H}, 0,-1.3814763135,1.573936493,-2.5950340773$ $\mathrm{H}, 0,-1.380729186,1.57526966,2.5946497588$ C, $0,1.63037824,-0.9074378746,-3.0378093774$ C, $0,1.6309613049,-0.906235167,3.037990273$ $\mathrm{H}, 0,1.6529417704,-1.8952503743,-2.5605145531$ $H, 0,1.6533151921,-1.8942698419,2.5611459718$ C, $0,1.2682007248,1.3526380563,-0.0003577385$ $0,0,2.0036579835,2.2507247919,0.0000331544$ C, $0,1.5625153238,-1.1049815068,4.5499237477$ C, $0,1.5615402312,-1.1068821785,-4.5496346571$ $\mathrm{H}, 0,0.6439289426,-1.5971539097,4.8760255483$ $\mathrm{H}, 0,0.642929545,-1.5993318113,-4.8752530412$ $\mathrm{H}, 0,1.6447193116,-0.1552834442,5.0828748293$ $\mathrm{H}, 0,1.6434555143,-0.1574128507,-5.083040023$ $\mathrm{H}, 0,2.4006818856,-1.7288138266,4.8761339829$ $\mathrm{H}, 0,2.3997015827,-1.7307451191,-4.8757989733$ C, $0,2.9166901906,-0.1903604634,2.6311083566$ C, $0,2.916144846,-0.1912556901,-2.6315919133$ $\mathrm{H}, 0,2.9606988956,0.828005115,3.0230938896$ $\mathrm{H}, 0,2.9599457469,0.826943618,-3.0240292336$ $\mathrm{H}, 0,3.0089018227,-0.143569349,1.5465717631$ $\mathrm{H}, 0,3.0086450108,-0.143981431,-1.5471024087$ $\mathrm{H}, 0,3.7821870253,-0.7318127865,3.0255194186$ $\mathrm{H}, 0,3.7815862895,-0.732796621,-3.0260031425$ C, $0,0.4226241248,2.3881201784,-3.3812005894$ C, $0,0.4235438684,2.3894521393,3.380436876$ $H, 0,1.2547183603,2.1265620603,-4.0393349128$ $\mathrm{H}, 0,1.2556074629,2.1280140264,4.0386577148$ $\mathrm{H}, 0,0.8343162042,2.6890518203,-2.4173347163$ $\mathrm{H}, 0,0.8352656633,2.6899348797,2.4164424701$ $\mathrm{H}, 0,-0.0725400484,3.2598429002,-3.8200428535$ $\mathrm{H}, 0,-0.0714637584,3.2614337309,3.8189403356$ C, $0,-1.1852138294,0.8809048948,-4.606043162$ C, $0,-1.1845250337,0.88298855,4.6059090858$ $\mathrm{H}, 0,-1.9029643068,0.0615692857,-4.5413768147$ $\mathrm{H}, 0,-1.9023935377,0.0637285339,4.5415867675$ $\mathrm{H}, 0,-0.4286019583,0.6089714863,-5.3422482483$ $\mathrm{H}, 0,-0.4279452113,0.6112497839,5.3422166123$ $\mathrm{H}, 0,-1.7211996338,1.7504890886,-5.0002965227$ $\mathrm{H}, 0,-1.7203856718,1.7528083113,4.9998111974$ $\mathrm{H}, 0,-3.837158938,2.0677164974,0.8165049301$ $\mathrm{H}, 0,-3.8368522645,2.0673603996,-0.8182087682$ $\mathrm{H}, 0,-5.5547290503,0.3011907179,-0.00059129$ $\mathrm{H}, 0,-4.0050134051,-0.4927742725,-1.0113866484$ $\mathrm{H}, 0,-4.0051618169,-0.492316731,1.0107981177$
$P, 0,-2.1712175771,-0.4035789971,0.2446507103$ C, $0,2.4538845374,0.7978737847,1.7264824174$ C, $0,-2.424979637,0.5104796678,1.8463828601$ $\mathrm{H}, 0,2.4649953055,1.8607912331,1.470657184$ $\mathrm{H}, 0,-2.5706116981,1.5675867039,1.6087122828$ $\mathrm{H}, 0,3.3900533343,0.5712392135,2.2415545992$ $\mathrm{H}, 0,-3.3024758712,0.1675312454,2.3988623809$ C,0,1.2581033181,0.5020281404,2.6100239629 C,0,-1.1615811992,0.3447013729,2.6669739095 $\mathrm{H}, 0,1.2692918575,1.138314558,3.5049012859$ $\mathrm{H}, 0,-1.2022046135,0.9636156051,3.5732731004$ $\mathrm{H}, 0,1.2724522584,-0.54054655524,2.9385702235$ $\mathrm{H}, 0,-1.03617444,-0.6965641704,2.9752622383$ C, $0,-3.3117359458,0.5103044458,-0.9437642611$ C, $0,3.2326837633,0.8131978261,-1.1048093833$ $\mathrm{H}, 0,-2.7173960967,1.4186464179,-1.0964403472$ $\mathrm{H}, 0,2.5339736828,1.6366649011,-1.291947536$ C, $0,-2.8818439037,-2.1037704428,0.632178731$ C, $0,3.1325120269,-1.7815564897,0.5463533458$ $\mathrm{H}, 0,-2.3519807366,-2.3218188241,1.5678887054$ $\mathrm{H}, 0,2.6665276989,-2.0292462655,1.5082722839$ C, $0,0.0393031075,-1.0592673769,-1.5615410444$ O,0,0.048068975,-1.5749585901,-2.6009327567 C,0,4.6425204913,-1.6953664655,0.7531743759 C, $0,-4.3824616078,-2.1802486011,0.9013982795$ $\mathrm{H}, 0,4.9367462131,-0.8868050421,1.4254978936$ $\mathrm{H}, 0,-4.7317257383,-1.4175322514,1.6003579257$ $\mathrm{H}, 0,5.1699789875,-1.5574696235,-0.1930391163$ $\mathrm{H}, 0,-4.9601150649,-2.0844739614,-0.0203466627$ $\mathrm{H}, 0,5.006863452,-2.6309026843,1.1890411484$ $\mathrm{H}, 0,-4.6281789514,-3.155638933,1.3329358208$ C,0,2.7687467082,-2.9044899957,-0.4222533365 C, $0,-2.4472912419,-3.1622291898,-0.3788595921$ $H, 0,3.1677628563,-2.7278842802,-1.4232065533$ H, $0,-2.9014592923,-3.0034989269,-1.3592398923$ $\mathrm{H}, 0,1.6877534908,-3.0168930661,-0.499322304$ $\mathrm{H}, 0,-1.3645852963,-3.1650555823,-0.499916066$ $\mathrm{H}, 0,3.1866757819,-3.8506895389,-0.0648777066$ $\mathrm{H}, 0,-2.755251394,-4.1534068233,-0.0317949296$ C, $0,-3.4261497948,-0.1988066001,-2.2919895988$ C, $0,3.4049736957,0.0586797598,-2.4222907988$ $\mathrm{H}, 0,-4.031213384,-1.105491742,-2.2165679206$ $\mathrm{H}, 0,4.1183010484,-0.7626800465,-2.3215869171$ $\mathrm{H}, 0,-2.4523359543,-0.4744555871,-2.6980904026$ $\mathrm{H}, 0,2.4641595455,-0.3516576034,-2.7904837681$ $\mathrm{H}, 0,-3.9185020191,0.4561727158,-3.0173788548$ $\mathrm{H}, 0,3.7973424639,0.7339728245,-3.1886146757$ C, $0,-4.6844378976,0.9317952794,-0.4213327024$ C, $0,4.5560375496,1.4165048312,-0.6357247542$ $\mathrm{H}, 0,-4.6292779695,1.4504829772,0.5373080033$ $\mathrm{H}, 0,4.4573383679,1.9782231608,0.2948366521$ $\mathrm{H}, 0,-5.359845164,0.0836625097,-0.307843004$ $\mathrm{H}, 0,5.3251614848,0.6576173306,-0.4907065113$ $\mathrm{H}, 0,-5.1480139103,1.6201693381,-1.1354759177$ $\mathrm{H}, 0,4.927388059,2.1113339027,-1.3960042601$ $\mathrm{H}, 0,1.0036229274,4.028908695,-0.9296121257$ $\mathrm{N}, 0,-0.881237297,4.0925768429,-1.5592303024$ $\mathrm{H}, 0,-0.2607435317,5.3320006271,0.9095103649$ $\mathrm{H}, 0,-1.1987009161,3.5729926461,1.2303946212$ $\mathrm{H}, 0,0.7964494422,3.7388343186,1.546093486$ $\mathrm{H}, 0,-1.1817100165,3.2748317882,-2.0818161303$ $\mathrm{H}, 0,-1.6689436349,4.4166825244,-1.008104398$ 


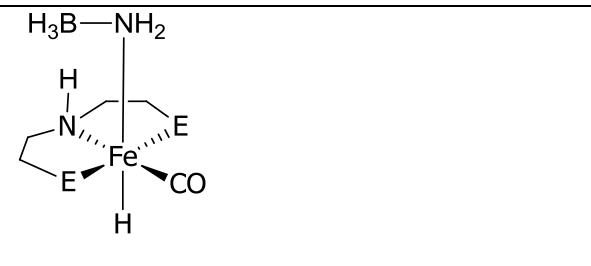

$\mathrm{H}, 0,-2.4836880373,1.0704353712,0.0017029655$ $\mathrm{N}, 0,-2.2138474653,0.0772867102,0.0054551417$ $\mathrm{Fe}, 0,-0.1177577619,0.2577129326,-0.0006601129$ $\mathrm{N}, 0,-0.3158791038,2.3462767495,-0.0096306394$ B, $0,-1.7252957286,3.0813491082,-0.0095243771$ $\mathrm{H}, 0,-2.3564330445,2.7405109666,-1.0092085415$ $\mathrm{H}, 0,-0.0888311792,-1.2699996228,0.0062063977$ $\mathrm{P}, 0,-0.3126495942,0.06290991,2.2319184681$ $P, 0,-0.3239659855,0.0426670742,-2.2303874852$ C, $0,-2.1541270959,0.0509502128,2.4606636636$ C, $0,-2.166579072,0.0288539114,-2.4496834466$ $\mathrm{H}, 0,-2.468930489,1.0923990289,2.5604215621$ $\mathrm{H}, 0,-2.4818362528,1.0693950372,-2.5571942211$ $\mathrm{H}, 0,-2.4684976228,-0.4892164407,3.3561789056$ $\mathrm{H}, 0,-2.4855208562,-0.5193125217,-3.3386972759$ C, $0,-2.7714182964,-0.5511195255,1.2151058831$ C, $0,-2.7775787209,-0.5619279045,-1.1956488513$ $\mathrm{H}, 0,-3.8638226912,-0.4389156354,1.2351826324$ $\mathrm{H}, 0,-3.8700635743,-0.4498027979,-1.2111913908$ $\mathrm{H}, 0,-2.5530403192,-1.6214179668,1.1566059247$ $\mathrm{H}, 0,-2.5589612542,-1.6316773311,-1.1286208697$ C, $0,0.2014883045,1.4573323453,-3.3540228674$ C, $0,0.2182779859,1.487776904,3.3400010236$ $\mathrm{H}, 0,-0.1781005731,2.3111004663,-2.7793480835$ $\mathrm{H}, 0,-0.1643526513,2.3362514656,2.7595326362$ C,0,0.2026219343,-1.6103034817,-2.9561145274 C, $0,0.2178285568,-1.5833379464,2.9699923029$ $\mathrm{H}, 0,-0.4611213769,-2.2824932183,-2.3980291649$ $\mathrm{H}, 0,-0.4486492904,-2.2606410957,2.4214283034$ C, $0,1.6100957884,0.2713081909,-0.0051037147$ $0,0,2.768927181,0.2638604469,-0.0080154108$ C, $0,-0.0289206141,-1.7740118543,4.4644242946$ C, $0,-0.0517184481,-1.8145721576,-4.4474761516$ $\mathrm{H}, 0,-1.0272490605,-1.4638544759,4.7784541768$ $\mathrm{H}, 0,-1.0516220144,-1.5072200037,-4.759245965$ $\mathrm{H}, 0,0.7000910481,-1.2243012576,5.0630645123$ $H, 0,0.6742593487,-1.2703954399,-5.0547955378$ $\mathrm{H}, 0,0.0802912269,-2.8325327987,4.7198288639$ $\mathrm{H}, 0,0.0561683225,-2.8753859951,-4.69376981$ C,0,1.6478723496,-1.968015702,2.5982006109 C, $0,1.6345022918,-1.99176411,-2.5880943184$ $\mathrm{H}, 0,2.3834018846,-1.3109920229,3.0669138091$ $\mathrm{H}, 0,2.3676961166,-1.3392611255,-3.0666819225$ $\mathrm{H}, 0,1.7998343655,-1.9367637634,1.520322135$ $H, 0,1.7920006305,-1.9505119898,-1.5113472418$ $\mathrm{H}, 0,1.8549493842,-2.9866495524,2.9401707024$ $\mathrm{H}, 0,1.8396921353,-3.013572203,-2.9216281191$ C, $0,1.720452393,1.5874336117,-3.4516364925$ C, $0,1.7377007044,1.6189097757,3.4287066346$ $\mathrm{H}, 0,2.1482391352,0.8035079573,-4.081198282$ $\mathrm{H}, 0,2.1687771645,0.8408352007,4.0632650905$ $\mathrm{H}, 0,2.2075122333,1.5361990557,-2.4766456884$ $\mathrm{H}, 0,2.2198162865,1.558766601,2.4517689624$ $\mathrm{H}, 0,1.9844517619,2.5463900592,-3.9070009689$ $\mathrm{H}, 0,2.0038842451,2.5820286697,3.8739011541$ C, $0,-0.4516765279,1.5365308534,-4.7342114176$ C, $0,-0.4278831348,1.5794357718,4.7227060881$ $\mathrm{H}, 0,-1.5377178734,1.4389096051,-4.6918619521$ $\mathrm{H}, 0,-1.5141116644,1.4813282423,4.686759622$

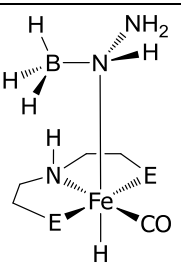

$\mathrm{H}, 0,-1.4429553177,2.1559471237,0.1197764347$ $\mathrm{N}, 0,-1.7590395861,1.1809194618,0.037871808$ $\mathrm{Fe}, 0,0.1059311229,0.2040298618,0.029060283$ $\mathrm{N}, 0,1.0808107118,2.083198514,0.1482861377$ $B, 0,0.249327916,3.4225509726,0.2617835141$ $\mathrm{H}, 0,1.0296843788,4.3625926987,0.351354865$ $\mathrm{H}, 0,-0.6972102138,-1.0901186307,0.014051813$ $P, 0,-0.2279984729,0.0299505534,2.253520994$ $\mathrm{P}, 0,-0.2023309175,0.1084653542,-2.2189212305$ C, $0,-1.8163861311,0.9635560019,2.4863384177$ C, $0,-1.6554056816,1.2428812596,-2.4147305249$ $\mathrm{H}, 0,-1.5494800231,2.0051680984,2.6770135821$ $\mathrm{H}, 0,-1.2567055775,2.2602569881,-2.4353795161$ $\mathrm{H}, 0,-2.4012956863,0.5997898099,3.3336631446$ $\mathrm{H}, 0,-2.2120358165,1.0726838911,-3.3387157026$ C, $0,-2.6082477682,0.8643823237,1.1983988433$ C, $0,-2.5449633333,1.06605308,-1.2029881721$ $\mathrm{H}, 0,-3.4773719253,1.5351069808,1.2309654016$ $\mathrm{H}, 0,-3.356780186,1.8053165486,-1.2070413811$ $\mathrm{H}, 0,-2.9841350863,-0.1531135053,1.0578483747$ $\mathrm{H}, 0,-3.0088507799,0.0752410883,-1.2082493727$ C, $0,1.0372628036,0.7851581311,-3.4523458668$ C, $0,0.9299565123,0.8926467185,3.4619252641$ $\mathrm{H}, 0,1.4143537735,1.6345900534,-2.8698078942$ $\mathrm{H}, 0,1.0638103136,1.8551484697,2.9521012655$ C, $0,-0.8762971355,-1.5412265599,-2.8295159846$ C, $0,-0.6625729778,-1.6908573174,2.8785339633$ $\mathrm{H}, 0,-1.7819101115,-1.6324688682,-2.218014388$ $\mathrm{H}, 0,-1.5662091657,-1.8837647105,2.2870859231$ C, $0,1.5657996523,-0.7200699795,-0.0023446082$ $0,0,2.5320963655,-1.3602567352,-0.0342985911$ C,0,-1.0238855647,-1.808077058,4.3574851686 C, $0,-1.2825145598,-1.5967284843,-4.3003288614$ $\mathrm{H}, 0,-1.7191933385,-1.0367328654,4.6937223459$ $\mathrm{H}, 0,-1.9158618575,-0.7598655417,-4.6006294188$ $\mathrm{H}, 0,-0.1356131118,-1.7623663489,4.9905231851$ $\mathrm{H}, 0,-0.4101332484,-1.6124016105,-4.9565030682$ $\mathrm{H}, 0,-1.4975833353,-2.7774730557,4.540276966$ $\mathrm{H}, 0,-1.8447727396,-2.5164037973,-4.4904907123$ C, $0,0.3637795725,-2.74945858884,2.4830711393$ C, $0,0.0232301596,-2.7207926081,-2.4656674981$ $H, 0,1.3199450246,-2.6028634557,2.9896521431$ $\mathrm{H}, 0,0.9654212749,-2.7022265906,-3.0163434144$ $\mathrm{H}, 0,0.5430025664,-2.7476923669,1.4092823659$ $\mathrm{H}, 0,0.2491012513,-2.7299620757,-1.399996417$ $\mathrm{H}, 0,-0.0052473269,-3.7408602841,2.7628186981$ $\mathrm{H}, 0,-0.482033906,-3.6595468517,-2.7129844543$ C, $0,2.2094177674,-0.1674773632,-3.6825271212$ C,0,2.2919857356,0.2050683417,3.5433093535 $\mathrm{H}, 0,1.9272736693,-1.0220207871,-4.3029988239$ $\mathrm{H}, 0,2.2334772959,-0.7302011884,4.1051150707$ $\mathrm{H}, 0,2.6236478149,-0.5521893703,-2.7488129582$ $\mathrm{H}, 0,2.703515115,-0.0231486781,2.5584453823$ $\mathrm{H}, 0,3.0123079403,0.3597293041,-4.2066354857$ $\mathrm{H}, 0,3.0061342528,0.8502614716,4.0631581559$ C, $0,0.4800336331,1.3107600879,-4.7738991007$ C, $0,0.3856416433,1.2136647633,4.8536919703$ $\mathrm{H}, 0,-0.3242680305,2.0339860613,-4.6303680668$ 
$\mathrm{H}, 0,-0.0676201912,0.7769106371,-5.4153023469$ $\mathrm{H}, 0,-0.0402635994,0.8260719458,5.4087096035$ $\mathrm{H}, 0,-0.2328748678,2.5122924043,-5.1789479248$ $\mathrm{H}, 0,-0.2069713299,2.5592167903,5.1574521942$ $\mathrm{H}, 0,-1.5787146524,4.2930129183,-0.0151235137$ $\mathrm{H}, 0,-2.3518546412,2.7492465853,0.9959807491$ $\mathrm{H}, 0,0.2147363814,2.7000148025,0.7793130403$ $H, 0,0.2108783869,2.6929146967,-0.804289402$

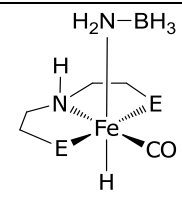

$\mathrm{H}, 0,-1.538024898,2.1062980563,0$. $\mathrm{N}, 0,-1.8318427738,1.1306445914,0$. $\mathrm{Fe}, 0,0.0273424078,0.1348511442,0$. $\mathrm{N}, 0,0.892028354,2.0348197444,0$. B,0,2.4912221072,2.2205671401,0. $\mathrm{H}, 0,2.7460702571,3.4220619639,0$. $\mathrm{H}, 0,-0.761290831,-1.1797099594,0$. $P, 0,-0.2224977643,0.0492788816,2.2266463531$ $P, 0,-0.2224977643,0.0492788816,-2.2266463531$ C, $0,-1.7505547872,1.0967279345,2.4461814753$ C, $0,-1.7505547872,1.0967279345,-2.4461814753$ $\mathrm{H}, 0,-1.433894815,2.1421310103,2.5192311985$ $\mathrm{H}, 0,-1.433894815,2.1421310103,-2.5192311985$ $\mathrm{H}, 0,-2.303222732,0.8661611027,3.3592973288$ $\mathrm{H}, 0,-2.303222732,0.8661611027,-3.3592973288$ C, $0,-2.6251964584,0.905279094,1.2212536468$ C, $0,-2.6251964584,0.905279094,-1.2212536468$ $\mathrm{H}, 0,-3.4953916326,1.5751889221,1.2499195274$ $\mathrm{H}, 0,-3.4953916326,1.5751889221,-1.2499195274$ $\mathrm{H}, 0,-2.9991863335,-0.1201165649,1.173448748$ H, $,-2.9991863335,-0.1201165649,-1.173448748$ C, $0,1.0508513743,0.862251493,-3.342231368$ C, $0,1.0508513743,0.862251493,3.342231368$ $\mathrm{H}, 0,1.3931540041,1.6846612951,-2.7036313128$ $\mathrm{H}, 0,1.3931540041,1.6846612951,2.7036313128$ C, $0,-0.7707227145,-1.593558722,-2.9604175352$ C, $0,-0.7707227145,-1.593558722,2.9604175352$ H, $0,-1.7233763582,-1.7416407765,-2.4367211567$ $\mathrm{H}, 0,-1.7233763582,-1.7416407765,2.4367211567$ C, $0,1.5029904143,-0.7888099947,0$.

$0,0,2.4363348335,-1.4650284113,0$.

C, $0,-1.0428651649,-1.5998502296,4.4628488767$ C, $0,-1.0428651649,-1.5998502296,-4.4628488767$ $\mathrm{H}, 0,-1.6926011197,-0.782977184,4.7838568858$ $\mathrm{H}, 0,-1.6926011197,-0.782977184,-4.7838568858$ $\mathrm{H}, 0,-0.1166163356,-1.5398130101,5.0373828301$ $\mathrm{H}, 0,-0.1166163356,-1.5398130101,-5.0373828301$ $\mathrm{H}, 0,-1.5352907936,-2.5365691927,4.7416400276$ $\mathrm{H}, 0,-1.5352907936,-2.5365691927,-4.7416400276$ C, $0,0.140907542,-2.750465442,2.5568495015$ C, $0,0.140907542,-2.750465442,-2.5568495015$ $\mathrm{H}, 0,1.1312253978,-2.6675346863,3.0075163186$ $\mathrm{H}, 0,1.1312253978,-2.6675346863,-3.0075163186$ $\mathrm{H}, 0,0.2608916473,-2.7963968254,1.4751769616$ $\mathrm{H}, 0,0.2608916473,-2.7963968254,-1.4751769616$ $\mathrm{H}, 0,-0.2959043668,-3.6961704302,2.8918036141$ $\mathrm{H}, 0,-0.2959043668,-3.6961704302,-2.8918036141$ C, $0,2.2671618845,-0.0378938993,-3.5565337749$ C, $0,2.2671618845,-0.0378938993,3.5565337749$
$\mathrm{H}, 0,-0.5905826199,1.6999420408,4.8201607131$ $\mathrm{H}, 0,0.1113171424,0.5164690183,-5.4247792711$ $\mathrm{H}, 0,0.3015627769,0.3241830626,5.4780038361$ $\mathrm{H}, 0,1.279900961,1.8272930111,-5.3142985235$ $\mathrm{H}, 0,1.0713304787,1.9018620967,5.3576754621$ $\mathrm{H}, 0,-0.4259705034,3.3601505642,1.2844800696$ $\mathrm{H}, 0,-0.4696050515,3.5724233227,-0.718459067$ $\mathrm{H}, 0,1.5655977855,2.01811005,1.0391109113$ $\mathrm{N}, 0,2.1045262328,2.2707009503,-0.8599952495$ $\mathrm{H}, 0,2.5298107995,3.1820948319,-0.6935022813$ $\mathrm{H}, 0,2.8179447438,1.5588007153,-0.7325388402$

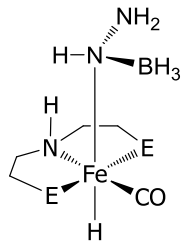

$\mathrm{H}, 0,-1.4250392609,2.1329265232,-0.1470462061$ $\mathrm{N}, 0,-1.7740223334,1.1634714263,-0.0385854923$ $\mathrm{Fe}, 0,0.0821257446,0.1970296124,-0.0205617069$ $\mathrm{N}, 0,0.9157873178,2.1475103957,0.1148661557$ B, $0,2.4634368123,2.4109276366,-0.1099815808$ $\mathrm{H}, 0,2.6734566358,3.5955158922,0.1647396135$ $\mathrm{H}, 0,-0.6759771284,-1.124761914,-0.0371054944$ $\mathrm{P}, 0,-0.2032749017,0.0716416939,2.2010109564$ $P, 0,-0.1950546045,0.0429833965,-2.243654605$ C, $0,-1.6618570834,1.2116579147,2.4172945056$ C,0,-1.7822202533,0.9920809367,-2.4846815124 $\mathrm{H}, 0,-1.2791057674,2.2357284914,2.4645644733$ $\mathrm{H}, 0,-1.5303008932,2.0423581892,-2.647894953$ $\mathrm{H}, 0,-2.2180736843,1.0322745853,3.3400446487$ $\mathrm{H}, 0,-2.3486788602,0.6528687822,-3.3544255174$ C, $0,-2.5560625175,1.0482268358,1.2013703556$ C, $0,-2.6021939787,0.8532455846,-1.2146866694$ $\mathrm{H}, 0,-3.3692034183,1.7868354438,1.2167000352$ $\mathrm{H}, 0,-3.4820866344,1.5107931008,-1.2521665328$ $\mathrm{H}, 0,-3.0210961583,0.058010942,1.2070486967$ $\mathrm{H}, 0,-2.9636634678,-0.1728256256,-1.1032877635$ C, $0,1.0173422412,0.8413877958,-3.4390941827$ C, $0,1.1021107367,0.7727408905,3.363600439$ $\mathrm{H}, 0,1.3458678024,1.7087015502,-2.8547698808$ $\mathrm{H}, 0,1.5198124716,1.5901757529,2.7641080253$ C, $0,-0.6800382083,-1.6599290798,-2.8867022444$ C, $0,-0.8528242928,-1.5556791144,2.8815750021$ $\mathrm{H}, 0,-1.6106325815,-1.822502512,-2.3289920979$ $\mathrm{H}, 0,-1.7893108421,-1.6454206715,2.3174355402$ C, $0,1.5794146377,-0.6923497792,-0.0205780489$ $0,0,2.526460288,-1.3511854902,-0.0196401569$ C, $0,-1.1865154936,-1.58103132,4.3711617073$ C, $0,-0.9947389048,-1.7476530446,-4.3784621902$ $\mathrm{H}, 0,-1.8018686707,-0.7357452233,4.6857836989$ $\mathrm{H}, 0,-1.6789672675,-0.9680321847,-4.7193566898$ $\mathrm{H}, 0,-0.2835900867,-1.5888865666,4.9846246163$ $\mathrm{H}, 0,-0.0880456145,-1.6895599926,-4.9835895089$ $\mathrm{H}, 0,-1.7422610796,-2.4940910512,4.6057695637$ $\mathrm{H}, 0,-1.4649620639,-2.7116773316,-4.5959041264$ C, $0,0.0169194716,-2.7473967659,2.485934174$ C, $0,0.2879351945,-2.7625793289,-2.464508899$ $\mathrm{H}, 0,0.9794798372,-2.740429661,2.9998242834$ $\mathrm{H}, 0,1.2610832606,-2.6627073702,-2.9480111199$ $\mathrm{H}, 0,0.2017479934,-2.7582007884,1.4122144417$ $\mathrm{H}, 0,0.441573637,-2.760900856,-1.3865103119$ $\mathrm{H}, 0,-0.4919267291,-3.6782638598,2.7538811336$ H,0,-0.1213166986,-3.7372465177,--2.7475951077 
$\mathrm{H}, 0,2.0352756446,-0.8883886061,-4.2021989698$ $\mathrm{H}, 0,2.0352756446,-0.8883886061,4.2021989698$ $\mathrm{H}, 0,2.6697031646,-0.4084680576,-2.6137568623$ $\mathrm{H}, 0,2.6697031646,-0.4084680576,2.6137568623$ $\mathrm{H}, 0,3.061270589,0.5366835042,-4.0405350325$ $\mathrm{H}, 0,3.061270589,0.5366835042,4.0405350325$ C, $0,0.5517682037,1.4500270922,-4.6613819318$ C, $0,0.5517682037,1.4500270922,4.6613819318$ $\mathrm{H}, 0,-0.3060052649,2.1134332939,-4.5342202453$ $\mathrm{H}, 0,-0.3060052649,2.1134332939,4.5342202453$ $\mathrm{H}, 0,0.2762729834,0.6779050682,-5.380985552$ $\mathrm{H}, 0,0.2762729834,0.6779050682,5.380985552$ $\mathrm{H}, 0,1.3527697834,2.0437057285,-5.1122997077$ $\mathrm{H}, 0,1.3527697834,2.0437057285,5.1122997077$ $\mathrm{H}, 0,2.9393374736,1.6841383016,-1.0025238314$ $\mathrm{H}, 0,2.9393374736,1.6841383016,1.0025238314$ $\mathrm{H}, 0,0.557266931,2.5718591112,-0.7977312221$ $\mathrm{H}, 0,0.557266931,2.5718591112,0.7977312221$
C,0,2.2657003826,-0.0178870097,-3.6353085004 C, $0,2.2482353862,-0.2125196297,3.5928562906$ $\mathrm{H}, 0,2.0591243386,-0.9042358661,-4.239773086$ $\mathrm{H}, 0,1.9456223795,-1.0411945796,4.2371697752$ $\mathrm{H}, 0,2.6965308067,-0.3342742813,-2.6848750106$ $H, 0,2.634086064,-0.6202232634,2.6581282775$ $\mathrm{H}, 0,3.0288740748,0.5653944631,-4.1576435467$ $\mathrm{H}, 0,3.0754421167,0.302290538,4.0888716876$ C, $0,0.4608473842,1.3377180362,-4.7723579637$ C, $0,0.6033739276,1.3668262378,4.6801836907$ $\mathrm{H}, 0,-0.4160539576,1.977496715,-4.6538784566$ $\mathrm{H}, 0,-0.1816294671,2.1116325722,4.536445162$ $\mathrm{H}, 0,0.1904028284,0.5182735458,-5.4392829699$ $\mathrm{H}, 0,0.2230006321,0.6031808738,5.359670458$ $H, 0,1.2267131123,1.9313825723,-5.2810795248$ $\mathrm{H}, 0,1.4345794834,1.8681149116,5.1854629202$ $\mathrm{H}, 0,2.7320114215,2.1925212446,-1.2832355981$ $\mathrm{H}, 0,3.0852155172,1.6729743544,0.6359755934$ $\mathrm{N}, 0,0.1115290383,3.1609913064,-0.5669654723$ $\mathrm{H}, 0,0.750492052,2.3716997276,1.0906641034$ $\mathrm{H}, 0,0.5275011974,4.0614145068,-0.3186660991$ $\mathrm{H}, 0,0.3585791784,3.0601179007,-1.5485991971$

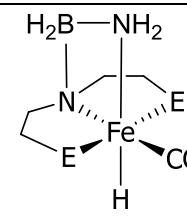

$\mathrm{N}, 0,0.0333995013,0.228718965,0.0006593948$ $\mathrm{Fe}, 0,2.1144965215,0.1475943463,0.0004898504$ $\mathrm{N}, 0,1.6000107932,2.149372176,0.0005873025$ B, $0,0.0591182421,1.830589638,0.0015615907$ $\mathrm{H}, 0,2.0917786882,-1.3720896256,0.0005315693$ $P, 0,1.9291365582,-0.1469040029,2.2116965731$ $P, 0,1.9289206507,-0.1463586412,-2.2107874405$ C, $0,0.0988775219,0.0111579428,2.4753569799$ C, $0,0.0988232848,0.0136487504,-2.4741930223$ $\mathrm{H}, 0,-0.103406416,1.0584920816,2.7033731644$ $\mathrm{H}, 0,-0.1025515973,1.0614906176,-2.7007222441$ H, $0,-0.263591834,-0.5933861595,3.3103969707$ $\mathrm{H}, 0,-0.264330567,-0.589336291,-3.3100601721$ C, $0,-0.5981989986,-0.3742235365,1.1844119662$ C, $0,-0.5984513394,-0.372798277,-1.1836847698$ $\mathrm{H}, 0,-1.6565982286,-0.0790570936,1.2379955829$ $\mathrm{H}, 0,-1.6567172264,-0.0771171043,-1.2369616032$ $\mathrm{H}, 0,-0.5731124556,-1.4650779485,1.068132228$ $\mathrm{H}, 0,-0.5738367771,-1.4637870964,-1.0685417308$ C, $0,2.6368559051,1.1268949967,-3.4098784028$ C, $0,2.6358801651,1.1269179335,3.4109239879$ $\mathrm{H}, 0,2.4053849196,2.0568058583,-2.8754037519$ $\mathrm{H}, 0,2.4029181354,2.0566366243,2.8767696116$ C, $0,2.3058601372,-1.883019568,-2.8275615878$ C, $0,2.3078145413,-1.8832930398,2.8282319541$ $\mathrm{H}, 0,1.5689457401,-2.4497659262,-2.2451837919$ $\mathrm{H}, 0,1.5711923549,-2.4506166026,2.246041206$ C,0,3.846499189,0.1820711545,0.0003853013 $0,0,5.0065177628,0.1928210652,0.0003250069$ C,0,2.0536369609,-2.1482175794,4.3100986322 C, $0,2.0510523043,-2.1477058505,-4.3093657438$ $\mathrm{H}, 0,1.0873259659,-1.772134262,4.6510962656$ $\mathrm{H}, 0,1.0848838555,-1.7709728386,-4.6500517291$ $\mathrm{H}, 0,2.8307833551,-1.7042590207,4.935551536$ $\mathrm{H}, 0,2.8283030742,-1.7042549894,-4.9350499149$ $\mathrm{H}, 0,2.0696296231,-3.2265244258,4.4967842816$ $\mathrm{H}, 0,2.0662641411,-3.2260183504,-4.4960880261$ C,0,3.6889107718,-2.3809773025,2.4100999299

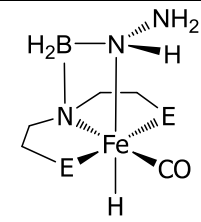

$\mathrm{N}, 0,0.0509108644,0.2411147566,-0.0240542801$ $\mathrm{Fe}, 0,2.1339780958,0.211361513,-0.0046305763$ $\mathrm{N}, 0,1.5794259315,2.1974723328,0.0570156208$ B, $0,0.0600029899,1.8155320104,0.1927217818$ $\mathrm{H}, 0,2.1669087628,-1.3080125902,0.0032681458$ $\mathrm{P}, 0,1.9152783417,-0.1738517532,2.1899369258$ $P, 0,1.9710467394,-0.0810573984,-2.2367103659$ C, $0,0.0725932064,-0.3030953113,2.4115945195$ C, $0,0.1862023888,0.3305733021,-2.4964626755$ $\mathrm{H}, 0,-0.273768182,0.6428014522,2.8254722793$ $\mathrm{H}, 0,0.1341709385,1.420523388,-2.5422244887$ $\mathrm{H}, 0,-0.2001862841,-1.0965296542,3.1113367682$ $\mathrm{H}, 0,-0.2271028335,-0.0774059872,-3.4216589258$ C, $0,-0.5657777496,-0.5469361186,1.0549353208$ C, $0,-0.5787755258,-0.1722645554,-1.2886090178$ $\mathrm{H}, 0,-1.6430134217,-0.3306847926,1.1149380085$ $\mathrm{H}, 0,-1.6143401131,0.1950770206,-1.3249868468$ $\mathrm{H}, 0,-0.4637939302,-1.606669998,0.7934202996$ $\mathrm{H}, 0,-0.6252589249,-1.2682714094,-1.3126590377$ C, $0,2.8670364431,1.0031909605,-3.4863794347$ C, $0,2.3873216245,1.1651805176,3.4330880186$ $\mathrm{H}, 0,2.8158409482,1.9620751243,-2.9634982178$ $\mathrm{H}, 0,1.9525205175,2.0446367268,2.9407713872$ C,0,2.0928275485,-1.8811497931,-2.7766869948 C, $0,2.5314777106,-1.8493394157,2.7856921935$ $\mathrm{H}, 0,1.3155551726,-2.3281593866,-2.1457166918$ $\mathrm{H}, 0,1.9030963082,-2.5047305234,2.1702063049$ C, $0,3.8632371445,0.310246117,-0.0058926755$ $0,0,5.0216994265,0.3735906692,-0.0207790548$ C, $0,2.2794632429,-2.1834823577,4.2540365421$ C,0,1.7525778391,-2.1748597403,-4.2359919794 $\mathrm{H}, 0,1.2659572495,-1.9441875212,4.5813004915$ $\mathrm{H}, 0,0.8196954932,-1.7084612093,-4.5582780073$ $\mathrm{H}, 0,2.9792275549,-1.6601746148,4.9086284689$ $\mathrm{H}, 0,2.5469538806,-1.8435868539,-4.9079754643$ $\mathrm{H}, 0,2.4312766661,-3.2552931016,4.4150402385$ H,0,1.643347669,-3.2549490536,-4.3765696279 
C,0,3.686659267,-2.3818508496,-2.4097877691 $\mathrm{H}, 0,4.4902803027,-1.8543930234,2.9321340241$ $\mathrm{H}, 0,4.488318492,-1.8561314723,-2.9322446348$ $H, 0,3.8414294286,-2.2605523221,1.3384610271$ $\mathrm{H}, 0,3.8396292965,-2.2612674583,-1.3382285222$ $H, 0,3.7859965392,-3.4442557076,2.6501766246$ $\mathrm{H}, 0,3.7826982564,-3.4452787645,-2.6496142635$ C, $0,4.1570707513,1.0341002406,-3.5316806968$ C, $0,4.1562583082,1.0359581148,3.5319809674$ $\mathrm{H}, 0,4.4558912969,0.1573815329,-4.1109237626$ $\mathrm{H}, 0,4.4563971842,0.1594208268,4.1108211819$ $\mathrm{H}, 0,4.6509462627,0.9819218763,-2.5609845524$ $\mathrm{H}, 0,4.6497196657,0.984612331,2.5610277134$ $\mathrm{H}, 0,4.5424919438,1.9133869606,-4.0566557439$ $H, 0,4.5409033994,1.9155526581,4.0570115006$ C,0,1.9797362019,1.2247176472,-4.7864609227 C, $0,1.9792868479,1.2236034636,4.7878304636$ $\mathrm{H}, 0,0.8926301655,1.2957905865,-4.729480328$ $\mathrm{H}, 0,0.8920263457,1.2927441096,4.7314195304$ $\mathrm{H}, 0,2.2298550249,0.3728045109,-5.4190825447$ $\mathrm{H}, 0,2.2312622198,0.372170995,5.42035902$ $H, 0,2.3384752194,2.1244138772,-5.2965533556$ $\mathrm{H}, 0,2.3366711922,2.1239556151,5.2977181803$ $\mathrm{H}, 0,-0.486438019,2.2672757277,-0.9945370584$ $\mathrm{H}, 0,-0.484678576,2.2657902224,0.9992355331$ $\mathrm{H}, 0,1.9110974598,2.6755722381,0.806296332$ $\mathrm{H}, 0,1.910104899,2.6754946457,-0.8055608988$
C,0,3.9823626664,-2.1302780818,2.4046951574 C, $0,3.4156721862,-2.5344246451,-2.3806379543$ $\mathrm{H}, 0,4.6799590247,-1.4877152227,2.945964737$ $\mathrm{H}, 0,4.2499782701,-2.1624779876,-2.9780200083$ $\mathrm{H}, 0,4.1469203319,-1.9884296658,1.3378747377$ $\mathrm{H}, 0,3.6391919174,-2.358053202,-1.3286264259$ $\mathrm{H}, 0,4.2336311229,-3.1662719142,2.6522024556$ $\mathrm{H}, 0,3.3562112022,-3.6156508597,-2.5397239327$ C, $0,4.3354269897,0.6209964686,-3.6634754205$ C,0,3.8988035367,1.3729868174,3.5102725705 $\mathrm{H}, 0,4.4461186656,-0.2945024349,-4.2495999962$ $\mathrm{H}, 0,4.3854953158,0.55494773,4.0465905204$ $\mathrm{H}, 0,4.8491766171,0.4780765143,-2.7118246421$ $\mathrm{H}, 0,4.3589877859,1.4470238828,2.5239448228$ $\mathrm{H}, 0,4.8590458761,1.4140171199,-4.2064393286$ $\mathrm{H}, 0,4.1233861811,2.2943154678,4.0562963045$ C,0,2.1767835996,1.1900632839,-4.8372723486 C, $0,1.7695649766,1.0923841128,4.8293385925$ $\mathrm{H}, 0,1.1428872903,1.5218208723,-4.7318085892$ $\mathrm{H}, 0,0.6927650762,0.9176550258,4.8054207375$ $\mathrm{H}, 0,2.1825777302,0.280778852,-5.4397271079$ $\mathrm{H}, 0,2.2252749527,0.3104660148,5.4368592866$ $\mathrm{H}, 0,2.7068636434,1.9601561338,-5.4074223018$ $\mathrm{H}, 0,1.9328249311,2.0424945419,5.3481587254$ $\mathrm{H}, 0,-0.6154836984,2.3888219679,-0.6424618541$ $\mathrm{H}, 0,-0.3232082452,2.098762115,1.3112612961$ $\mathrm{H}, 0,1.8982044056,2.6574177846,0.9029151783$ $\mathrm{N}, 0,1.8827112466,3.0483998801,-1.0637955529$ $\mathrm{H}, 0,1.3440262694,3.9083147619,-0.9773654067$ $\mathrm{H}, 0,2.8667275982,3.2957052147,-1.0147571047$

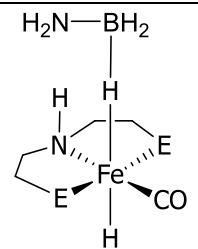

$\mathrm{H}, 0,0.8791965283,-1.1776085129,0.0007600701$ $\mathrm{Fe}, 0,0.0296306434,0.0794027419,0.0004215464$ $P, 0,-0.0368250717,-0.1486586241,2.2194745332$ $\mathrm{P}, 0,-0.0364196965,-0.1492569789,-2.2186008044$ C, $0,-1.6308798394,-1.0789527846,2.4460030136$ C, $0,-1.6307814673,-1.0790391429,-2.4451011629$ $\mathrm{H}, 0,-2.4311577603,-0.3385767259,2.5198345216$ $\mathrm{H}, 0,-2.4307805152,-0.3383636029,-2.5190080207$ $\mathrm{H}, 0,-1.6498571543,-1.681972637,3.3564325987$ $\mathrm{H}, 0,-1.6499967129,-1.6821024884,-3.3554931109$ C, $0,-1.8355783916,-1.9344282858,1.2098123788$ C, $0,-1.8357529968,-1.9343913064,-1.2088608771$ $\mathrm{H}, 0,-2.8358162365,-2.3904154256,1.2196645165$ $\mathrm{H}, 0,-2.8360902209,-2.3901657951,-1.218733666$ $\mathrm{H}, 0,-1.10584862,-2.7493186802,1.181665595$ $\mathrm{H}, 0,-1.1062242974,-2.7494614192,-1.1806819091$ $\mathrm{N}, 0,-1.6568439802,-1.1179578666,0.0004715566$ C, $0,-0.254340273,1.3690497399,-3.3006005795$ C, $0,-0.2555306077,1.369944034,3.3009057579$ $\mathrm{H}, 0,-0.9189027234,1.9587148978,-2.6582717527$ $\mathrm{H}, 0,-0.9201749658,1.9591267938,2.6582169374$ C,0,1.2318090361,-1.3172933267,-2.9713590231 C, $0,1.2316667207,-1.3159838698,2.9728876164$ $\mathrm{H}, 0,0.9840108709,-2.2455885459,-2.4415204544$ $\mathrm{H}, 0,0.9841929979,-2.2445811336,2.4434236993$ C, $0,1.447592504,1.0734103926,0.0003934407$ $0,0,2.4081068231,1.719407449,0.0003947368$ C, $0,1.1110546553,-1.5715592524,4.4730444231$

$\mathrm{H}_{2} \mathrm{NHN}-\mathrm{BH}_{2}$<smiles>CC1CC2CC1CC(=O)N2</smiles>

H $0.8668249825,-1.1340908682,0.0167115697$ Fe $-0.0047660858,0.1018689472,0.0097178981$ P $-0.0489832157,-0.113300431,2.2383702493$ P $-0.0727629669,-0.168909074,-2.2103558237$ C $-1.6758699706,-0.9797015678,2.4727220857$ C $-1.6133106523,-1.1836305546,-2.4127451509$ H $-2.4481666154,-0.206288433,2.486410636$

H - $2.436769994,-0.4679121095,-2.5046895296$ H -1.7399809143,-1.538564819,3.4088067029 H $-1.5937604401,-1.8207776823,-3.2995510485$ C $-1.8782513452,-1.885253728,1.2718943325$ C $-1.7933485042,-1.9998225245,-1.1469580862$ H $-2.8842241828,-2.3277422768,1.2890589888$ H $-2.770654365,-2.5036332386,-1.1526678138$ H $-1.1609005893,-2.7115437596,1.2875704897$ H -1.0258848244,-2.7758847883,-1.0675457785 N $-1.6749264945,-1.1253432846,0.0300516715$ C $-0.3845075662,1.3381486906,-3.2853914066$ C $-0.1655000083,1.3982342654,3.3472065204$ H $-1.0816484458,1.8839726618,-2.640260015$ H $-0.7803137245,2.0486098219,2.7164796295$ C $1.257571249,-1.26244227,-2.9661451772$ C $1.1789160359,-1.3433908574,2.9608534099$ H $1.0558941861,-2.2067079454,-2.4447447402$ H $0.8866927389,-2.2549458825,2.4251553659$ C $1.4039929365,1.1097019803,-0.0009225904$ O $2.359954728,1.7619925076,-0.0007014083$ C $1.0648886371,-1.612152785,4.4595776026$ 
C,0,1.1113548204,-1.573511045,-4.4714175176 $\mathrm{H}, 0,0.0921876944,-1.8079498845,4.7863079511$ $\mathrm{H}, 0,0.0924842928,-1.8098331193,-4.7847232283$ $\mathrm{H}, 0,1.451747699,-0.7120004579,5.053428803$ $\mathrm{H}, 0,1.4523121153,-0.7142858863,-5.0521402394$ $\mathrm{H}, 0,1.744068649,-2.4193837724,4.7531090295$ $\mathrm{H}, 0,1.744233024,-2.4215929341,-4.7510111684$ C,0,2.6641553751,-0.9522340946,2.5883301551 C, $0,2.6643442355,-0.9537736917,-2.5867434924$ $\mathrm{H}, 0,3.3469153157,-1.7409022418,2.9192009064$ $H, 0,3.3469125922,-1.7428771795,-2.9169710654$ $\mathrm{H}, 0,2.9884447777,-0.020889123,3.0566486223$ $\mathrm{H}, 0,2.9890336898,-0.0228396419,-3.0556028895$ $\mathrm{H}, 0,2.7666805037,-0.8454857694,1.5088962576$ $\mathrm{H}, 0,2.766684519,-0.8463685307,-1.5073548758$ C, $0,1.0420050211,2.1604725097,-3.4659173912$ C, $0,1.0404902232,2.1619138827,3.4661494962$ $\mathrm{H}, 0,1.7393388906,1.6585970761,-4.1414638941$ $\mathrm{H}, 0,1.7379030107,1.660543432,4.141990104$ $\mathrm{H}, 0,1.5495982317,2.3317331697,-2.516206656$ $\mathrm{H}, 0,1.5481644167,2.3330187199,2.516455284$ $\mathrm{H}, 0,0.8197485501,3.1395022797,-3.9005384584$ $\mathrm{H}, 0,0.8177928913,3.1410160882,3.900381268$ C, $0,-0.9540505632,1.1667167132,-4.6442138653$ C, $0,-0.955383088,1.1678557465,4.6444775914$ $\mathrm{H}, 0,-1.891165853,0.6143306959,-4.5530675079$ $\mathrm{H}, 0,-1.8922203865,0.6149818575,4.5534172264$ $\mathrm{H}, 0,-0.3238366181,0.6450519721,-5.3657192554$ $\mathrm{H}, 0,-0.3250370865,0.6467987257,5.3663075755$ $\mathrm{H}, 0,-1.1967375243,2.1444168003,-5.0723984177$ $\mathrm{H}, 0,-1.1986012475,2.145622575,5.0722079314$ $\mathrm{H}, 0,-0.9854740041,1.4039626718,0.0000566906$ $\mathrm{N}, 0,-3.3415760828,1.1457894914,0.0004818987$ B, $0,-2.140461185,2.0842213948,-0.0002793251$ $\mathrm{H}, 0,-3.9345959924,1.2406144958,-0.8127311672$ $\mathrm{H}, 0,-3.9356037387,1.243269716,0.8126337841$ $\mathrm{H}, 0,-2.027836145,2.742272622,1.0133861562$ $\mathrm{H}, 0,-2.0279630783,2.7410281457,-1.0147959295$ $\mathrm{H}, 0,-2.4063212945,-0.3751506535,0.000596932$<smiles>[B]N[C@H]1CC[C@@H](C=O)[C@@H]1F</smiles>

$\mathrm{N}, 0,-0.0543823909,-0.6629506594,-1.1580706413$ $\mathrm{P}, 0,-2.363730285,0.4362887124,-0.04569237$ $P, 0,2.0177019481,0.9658360408,-0.2270223951$ C, $0,2.4020277908,-0.5855691748,-1.15763756$ $\mathrm{H}, 0,2.6173024018,-1.3546523994,-0.409882096$ $\mathrm{H}, 0,3.2736197377,-0.5018107404,-1.809356751$ C, $0,1.1508912517,-0.9638549127,-1.9314161989$ $\mathrm{H}, 0,1.1877467857,-2.0352281339,-2.1781997169$ $\mathrm{H}, 0,1.122463009,-0.4330966761,-2.8971488254$ C, $0,-1.2104187026,-1.2785956624,-1.8041627441$ $\mathrm{H}, 0,-0.9969946204,-2.3305880626,-2.0488649731$ $\mathrm{H}, 0,-1.4167682794,-0.7889593729,-2.7703650052$ C, $0,-2.4417295379,-1.1983575447,-0.9153416977$ $\mathrm{H}, 0,-2.3906647008,-1.964727524,-0.1361250743$ $\mathrm{H}, 0,-3.3635207798,-1.3615350409,-1.4779907614$ C, $0,3.254107838,0.9837893617,1.1842151992$ C, $0,2.3837931287,2.3359801437,-1.4666100106$
C 1.1443160654,-1.5119921381,-4.4682365054 H $0.041917593,-1.8203182169,4.7794909228$ H $0.1372729661,-1.7983402401,-4.7777593582$ H $1.4379392931,-0.7708662255,5.0470468454$ H $1.436094659,-0.6302468763,-5.042363241$ H $1.6731014537,-2.4830255885,4.7236209824$ H 1.8197621201,-2.3229854927,-4.7582761844 C $2.6218084879,-1.03901234,2.5626848609$ C $2.6720866859,-0.8331168311,-2.5840249356$ H $3.2708472284,-1.8661867033,2.8663403599$ H 3.3920103331,-1.5791703285,-2.9346645249 H 2.997143175,- $0.1347734501,3.045357982$ H 2.9453057223,0.1226252647,-3.0355860763 H $2.7130736118,-0.914493252,1.4840488182$ H $2.7784359538,-0.7415308359,-1.5037749832$ C $0.8658455404,2.1990111378,-3.4557453244$ C $1.1855033137,2.0847140284,3.5432377023$ H 1.5913991058,1.730982208,-4.1260240054 H 1.8355881512,1.5169346966,4.2134098747 H 1.3614766457,2.4037302375,-2.5061496232 H 1.7153554614,2.238140676,2.6024691147 H $0.5912151349,3.1613551903,-3.8976239999$ H 1.0324825056,3.0677753546,3.9981828583 C $-1.0820121854,1.0979308226,-4.6229268466$ C $-0.8940972895,1.2174331497,4.6779984272$ H - $1.998168881,0.5157769142,-4.5140247369$ H $-1.8812948487,0.7682444436,4.5567283689$ H $-0.4378398688,0.5936505744,-5.3445403652$ H $-0.3283244281,0.6070623336,5.3832830485$ H $-1.3632452433,2.06269785,-5.0572760122$ H $-1.0418021033,2.197964125,5.1417157607$ H - $1.0354346245,1.4227468459,-0.1201161726$ N -3.374783574,1.1367813396,-0.1823696402 B $-2.1729086268,2.0296219295,0.1106008972$ N -3.821177923,1.190986273,-1.5482556125 H $-4.1322034021,1.391379203,0.4450327421$ H - $2.1799175899,2.3464677324,1.2805321373$ H $-2.064917179,2.986111585,-0.6392152231$ H $-2.4340511049,-0.3986583692,-0.0215333117$ H $-4.6800088623,0.6576323896,-1.635133933$ H -4.0270704616,2.1557021881,-1.805087471

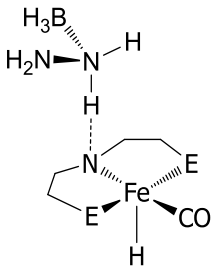

$\mathrm{H}, 0,1.4325910708,-0.0428954057,-0.0172644504$ $\mathrm{Fe}, 0,-0.0450603851,0.0988934398,-0.0361355067$ $\mathrm{P}, 0,-0.0364540441,-0.2014154945,2.1766778081$ $P, 0,0.0200407948,-0.1805355755,-2.2446071768$ C, $0,-1.612556655,-1.1482483718,2.384866564$ C, $0,-1.5723634662,-1.0874701378,-2.5025901872$ $\mathrm{H}, 0,-2.4118539896,-0.4062635087,2.4643940287$ $\mathrm{H}, 0,-2.3547582245,-0.3252760644,-2.5648915527$ $\mathrm{H}, 0,-1.6382024317,-1.765646672,3.2851291491$ $\mathrm{H}, 0,-1.6008265255,-1.6806854816,-3.4192192627$ C, $0,-1.8036037152,-1.9749555579,1.1243211612$ C, $0,-1.7901465434,-1.9423610149,-1.2641568324$ $\mathrm{H}, 0,-2.8643027543,-2.2528548889,1.0282430248$ $\mathrm{H}, 0,-2.8535789558,-2.2196800469,-1.1890550217$ $\mathrm{H}, 0,-1.2524089724,-2.9263631054,1.2053329549$ $\mathrm{H}, 0,-1.2461502798,-2.8964777629,-1.3625909506$ $\mathrm{N}, 0,-1.3625784612,-1.2358153518,-0.058458391$ 
C,0,-3.4613925219,0.2143154912,1.4642575554 C, $0,-3.1680542668,1.6124375751,-1.2772755504$ C, $0,-4.6264015993,1.3270833322,-1.6310759684$ $\mathrm{H}, 0,-2.8011071248,-0.3800047963,2.1072649618$ C, $0,3.8138808881,2.3981866408,-1.999951602$ $\mathrm{H}, 0,2.8218135135,0.2167501531,1.8375212576$ $\mathrm{Fe}, 0,-0.1622798358,0.6615293114,0.1597579847$ C, $0,-0.1391348971,1.0152742343,1.851182638$ $\mathrm{H}, 0,-0.3427625538,2.1288671849,0.0166646552$ $0,0,-0.1278513221,1.1973491722,2.9984963753$ C, $0,3.2505066517,2.3051504465,1.9511724889$ C, $0,4.6741620505,0.5396624048,0.8397459187$ C, $0,-3.7276825883,1.5361750347,2.1830066975$ C, $0,-4.7516091934,-0.5786071736,1.2625340018$ C, $0,-2.9718617431,3.085268398,-0.922211233$ $\mathrm{H}, 0,-2.5549275899,1.4088281063,-2.164577689$ $\mathrm{H}, 0,1.7245326219,2.0297019609,-2.2890863445$ C, $0,1.9186475229,3.715846724,-1.003897229$ $\mathrm{N}, 0,0.4695671136,-2.6881845419,1.4153403254$ $\mathrm{H}, 0,0.3395179379,-1.9513290464,0.7052750089$ $\mathrm{H}, 0,0.3256122467,-2.2520059316,2.3217283377$ $\mathrm{H}, 0,-0.2709804318,-3.3709046259,1.2867470269$ B, $0,1.9318491383,-3.4123864134,1.337887126$ $\mathrm{H}, 0,2.7504424326,-2.5473117268,1.5799090692$ $\mathrm{H}, 0,1.9233546827,-4.2840023875,2.181686835$ $H, 0,2.0306293758,-3.852241101,0.2099500563$ $\mathrm{H}, 0,-5.2104552108,-0.7801223,2.2356048383$ $\mathrm{H}, 0,-5.481133022,-0.0276534571,0.6679771805$ $\mathrm{H}, 0,-4.5813673987,-1.5415947836,0.7778549604$ $\mathrm{H}, 0,-4.1537180373,1.3419764648,3.1713300675$ $\mathrm{H}, 0,-2.8174762401,2.1204493416,2.3246041633$ $\mathrm{H}, 0,-4.446692412,2.1477845193,1.6336159944$ $\mathrm{H}, 0,3.7870382135,2.183310885,2.8963798183$ $\mathrm{H}, 0,3.7585564061,3.0926461006,1.3897485679$ $H, 0,2.2418024672,2.64811698855,2.1862468721$ $\mathrm{H}, 0,5.2516076052,0.4328173803,1.7630617529$ $\mathrm{H}, 0,4.694141365,-0.4266306848,0.3346841148$ $\mathrm{H}, 0,5.1927617889,1.2670663163,0.2135395958$ $\mathrm{H}, 0,1.9340720239,4.4125179426,-1.8475574248$ $\mathrm{H}, 0,0.9046060772,3.6823596743,-0.6051062096$ $\mathrm{H}, 0,2.574230127,4.1247148243,-0.2327011869$ $\mathrm{H}, 0,3.8543121886,3.0711479676,-2.8622464942$ $\mathrm{H}, 0,4.5012230731,2.795698244,-1.2504615068$ $\mathrm{H}, 0,4.1924295618,1.4280812684,-2.3261082623$ $\mathrm{H}, 0,-3.2641426288,3.7108732406,-1.7709086169$ $\mathrm{H}, 0,-3.5877110285,3.3817573448,-0.0709628854$ $\mathrm{H}, 0,-1.9311590104,3.2996421316,-0.6789346212$ $\mathrm{H}, 0,-4.9119006991,1.9136554202,-2.5097399891$ $\mathrm{H}, 0,-4.8133543554,0.2777571812,-1.865842281$ $\mathrm{H}, 0,-5.2977447578,1.6182070095,-0.8204055301$
C,0,-0.0847233499,1.2910988851,-3.4078580787 C, $0,-0.2082138816,1.2572335967,3.3454599155$ $\mathrm{H}, 0,-0.767643001,1.9411279446,-2.8481945647$ $\mathrm{H}, 0,-0.904453207,1.8859573169,2.778546985$ C,0,1.319442628,-1.4057201703,-2.8403922136 C, $0,1.2804791521,-1.399928133,2.7887590133$ $\mathrm{H}, 0,1.0629685754,-2.2716624722,-2.2167018389$ $\mathrm{H}, 0,1.0673567756,-2.2631634226,2.1452373393$ C, $0,0.3152933692,1.7894070897,-0.0283628641$ $0,0,0.5107261196,2.9343884262,-0.0328269152$ C,0,1.1559579686,-1.8478468581,4.2438252007 C, $0,1.2244001407,-1.828860723,-4.3049660226$ $\mathrm{H}, 0,0.1438839013,-2.1554458337,4.51189393$ $\mathrm{H}, 0,0.2124324892,-2.1054084309,-4.6057465105$ $H, 0,1.4623750097,-1.0564564618,4.930911787$ $\mathrm{H}, 0,1.571970165,-1.0367777034,-4.9715104497$ $\mathrm{H}, 0,1.8160150074,-2.7023884957,4.4228922721$ $\mathrm{H}, 0,1.8659529745,-2.6984421663,-4.4784104588$ C, $0,2.7069146127,-0.9474131599,2.4810262021$ C, $0,2.7483852772,-1.0003542781,-2.4828213566$ $\mathrm{H}, 0,3.4014153199,-1.7762091403,2.6489319557$ $\mathrm{H}, 0,3.4231931122,-1.8462735507,-2.6451463833$ $\mathrm{H}, 0,3.0178980812,-0.1256160116,3.1287986427$ $\mathrm{H}, 0,3.1029270952,-0.1768086303,-3.1055613668$ $\mathrm{H}, 0,2.8063095754,-0.6205417318,1.4457813312$ $\mathrm{H}, 0,2.8251202913,-0.6951331033,-1.4391579771$ C, $0,1.2484101131,2.0266291746,-3.53485536$ C, $0,1.0982661798,2.0328872284,3.5050842321$ $\mathrm{H}, 0,1.9493798395,1.472653115,-4.1633124785$ $\mathrm{H}, 0,1.8020533598,1.4991010712,4.1479420062$ $H, 0,1.7193218719,2.2000634044,-2.5662224192$ $\mathrm{H}, 0,1.5864542354,2.2264170595,2.5488090563$ $\mathrm{H}, 0,1.0906409738,3.0011658025,-4.0055903292$ $\mathrm{H}, 0,0.8979165402,2.9995550786,3.9757319585$ C, $0,-0.7135117617,1.0315006751,-4.7756670682$ C, $0,-0.8560239275,0.966411387,4.6978401029$ $\mathrm{H}, 0,-1.6894192705,0.5495051678,-4.6996297894$ $\mathrm{H}, 0,-1.8173216782,0.4610108331,4.596766994$ $\mathrm{H}, 0,-0.0772938519,0.4123179526,-5.4093437978$ $\mathrm{H}, 0,-0.2152737275,0.3607859913,5.3403282976$ $\mathrm{H}, 0,-0.860338593,1.9843718716,-5.293899389$ $\mathrm{H}, 0,-1.0417189082,1.9110778319,5.2181207586$ $\mathrm{N}, 0,-3.4381752788,1.2768191282,-0.0502119325$ $\mathrm{N}, 0,-4.213514496,0.9963033269,-1.245289$ $B, 0,-4.2884904039,1.4199176579,1.3030531271$ $\mathrm{H}, 0,-4.736002683,0.1553919989,-1.0073308992$ $\mathrm{H}, 0,-4.9252064941,1.7247607657,-1.2611435606$ $\mathrm{H}, 0,-4.8203097181,0.3388398854,1.4767061564$ $\mathrm{H}, 0,-3.4974680049,1.7049960309,2.1804547294$ $\mathrm{H}, 0,-5.089801869,2.3118572907,1.0983944624$ $\mathrm{H}, 0,-2.7103373168,0.5422897447,0.0031833841$ $\mathrm{H}, 0,-2.9161760941,2.1286063446,-0.2443125746$ 


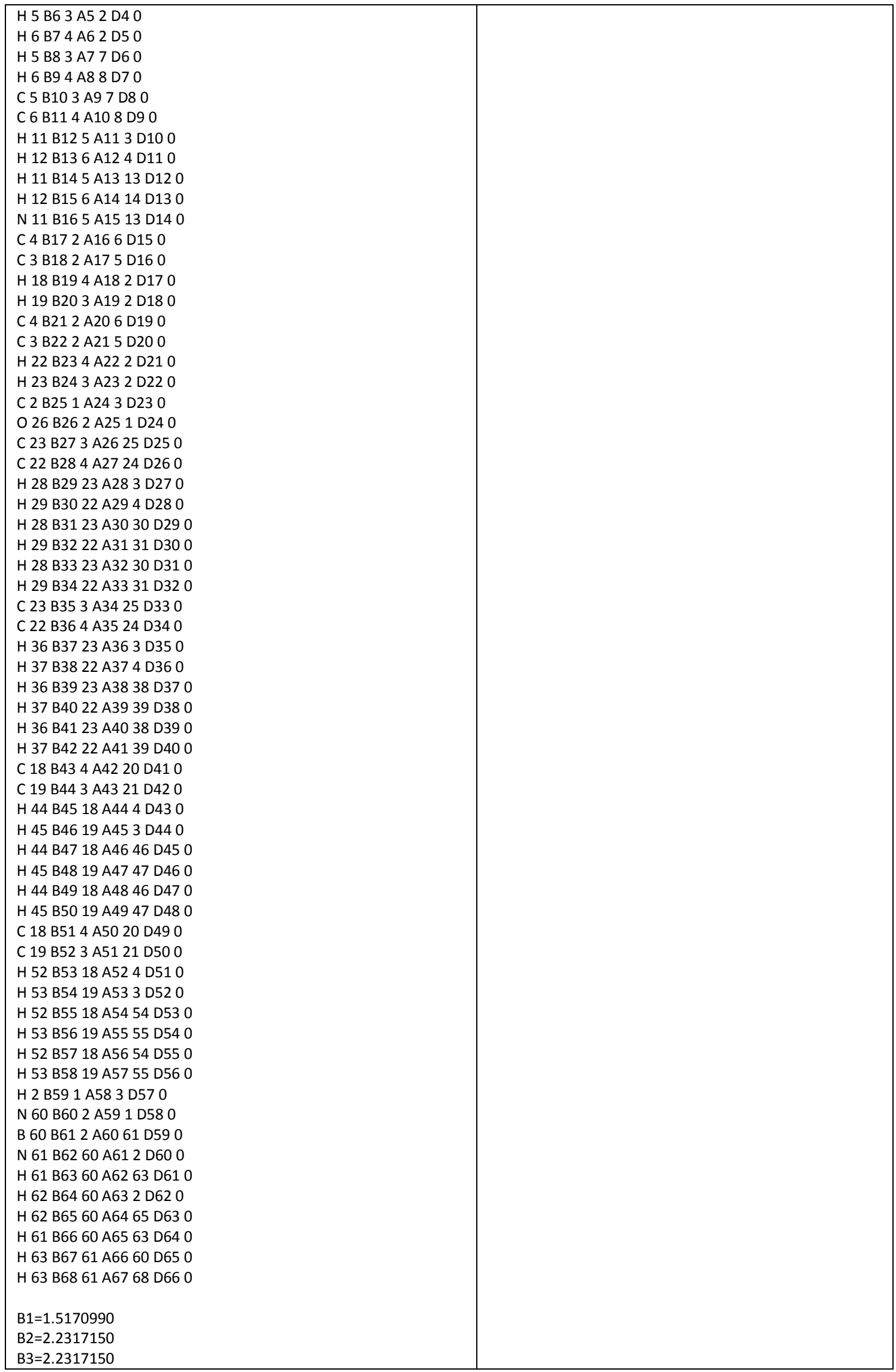




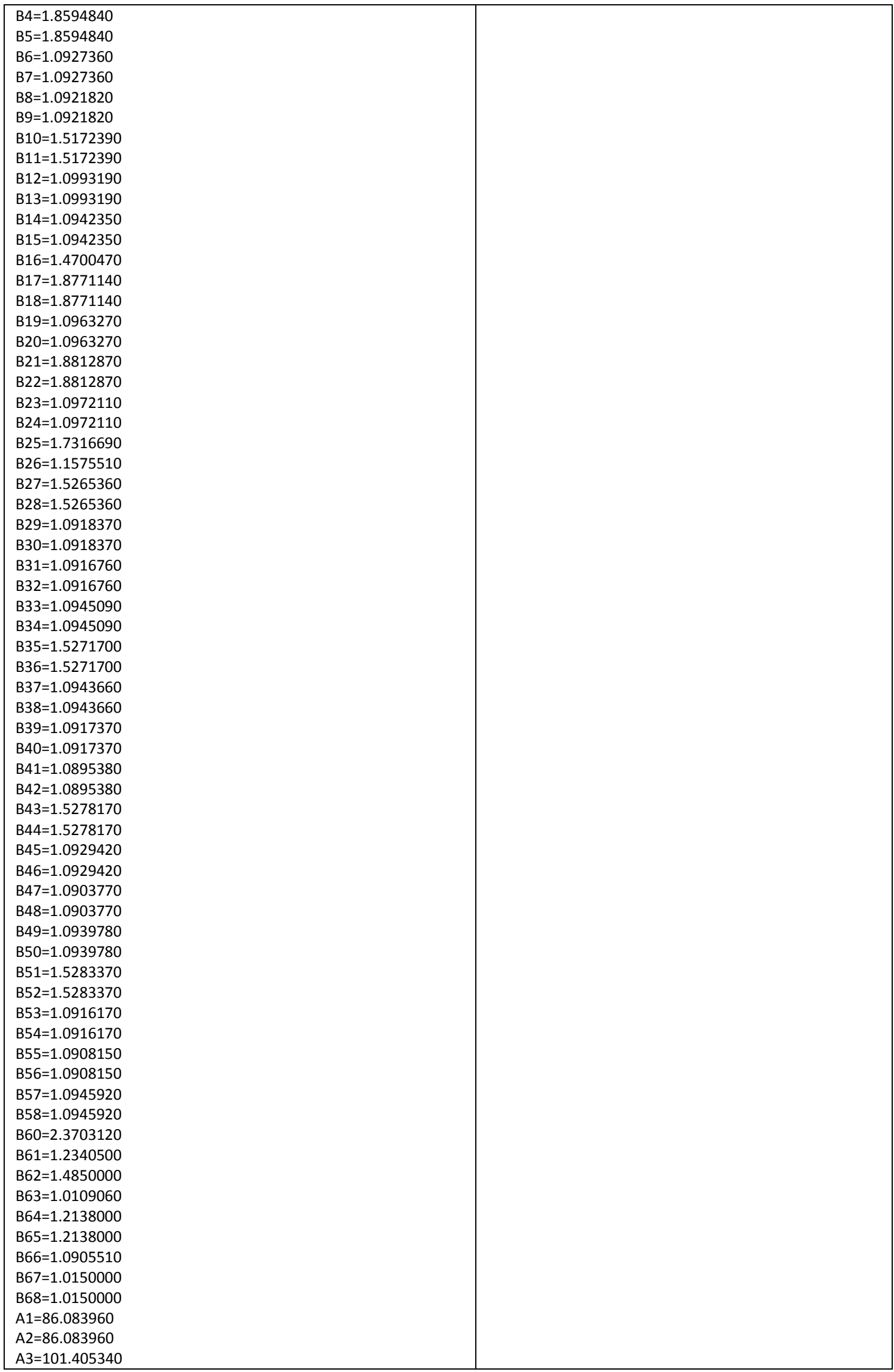




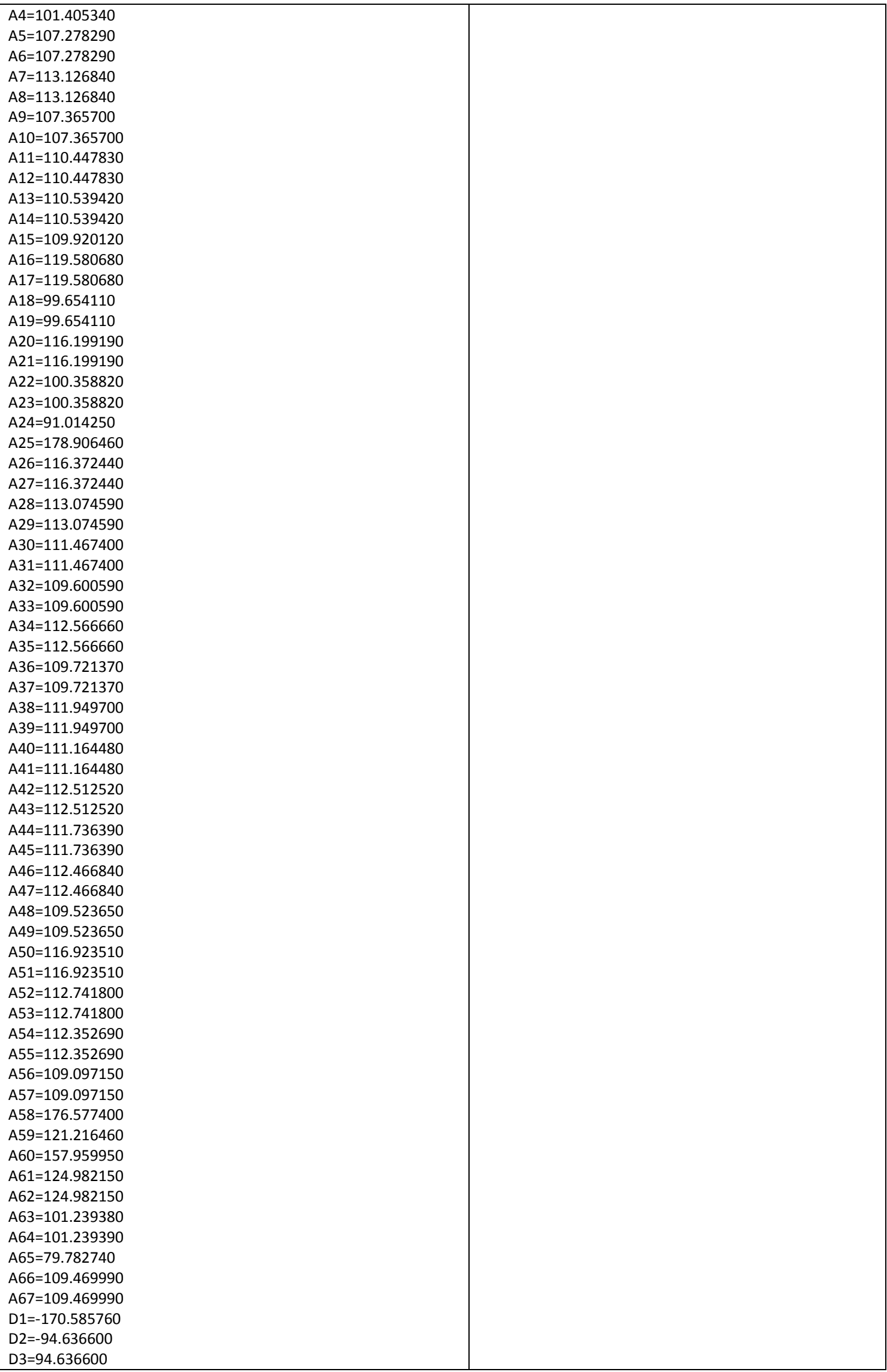




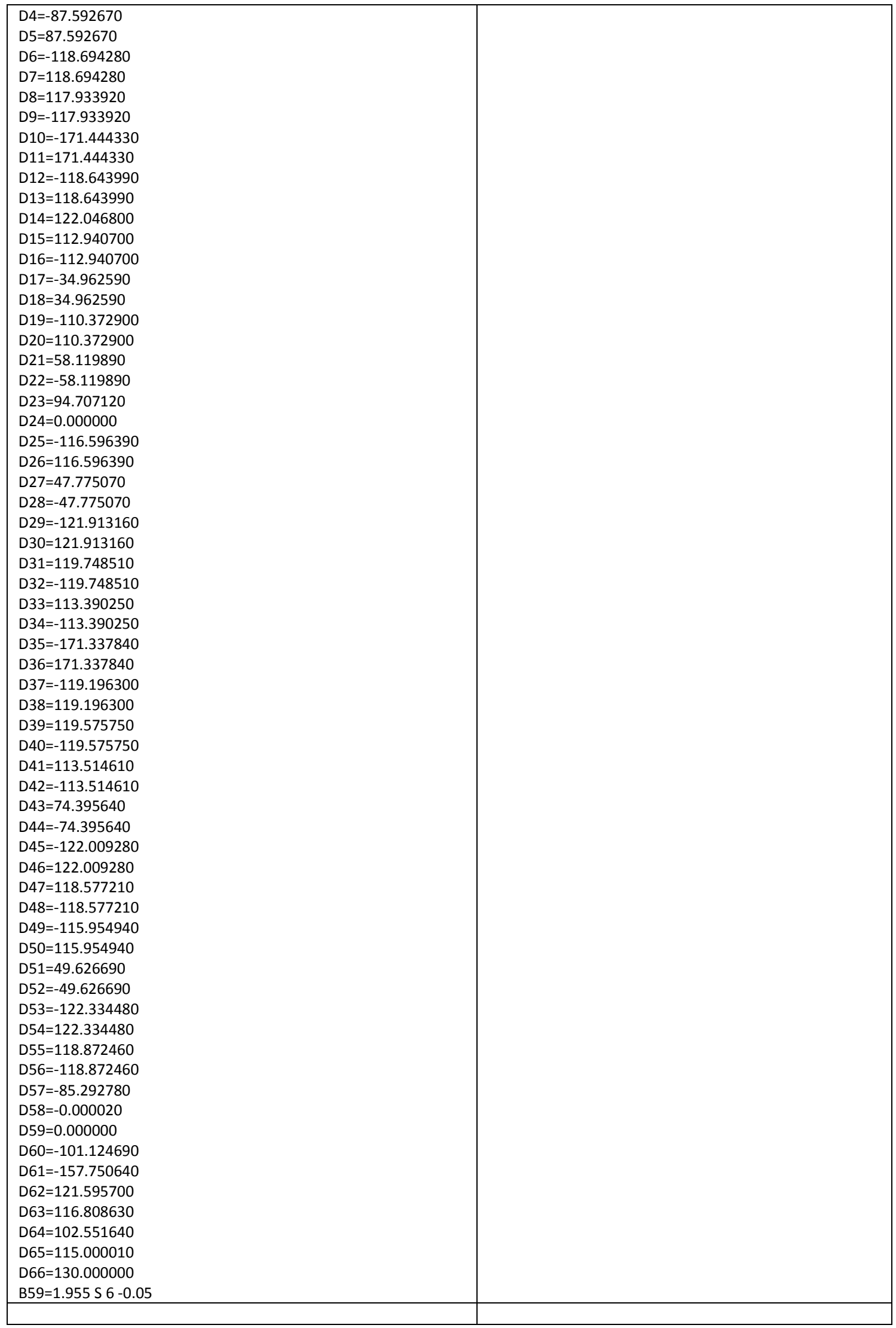


Table S3. B3PW91-SCRF-D3 optimized Cartesian coordinates (THF solution with dispersion)

\begin{tabular}{|c|c|}
\hline $\mathrm{N} 2 \mathrm{H} 4-\mathrm{BH} 3$ & $\mathrm{H} 2 \mathrm{~B}=\mathrm{NH}-\mathrm{NH} 2$ \\
\hline$H, 0,-0.1041850962,-0.1170615969,0.1475631093$ & B,0,-0.0347366902,1.116543834,1.6828016061 \\
\hline$B, 0,0.1812963663,-0.2186264607,1.3260501367$ & $H, 0,-0.1618657109,2.1662802537,1.1220355848$ \\
\hline$H, 0,0.0578788463,-1.3595240521,1.7305996462$ & $\mathrm{H}, 0,0.0672749135,1.0239962698,2.8713699744$ \\
\hline$H, 0,-0.4234341132,0.58191101,2.0145124153$ & $N, 0,-0.0021934929,-0.0502158389,0.9404176914$ \\
\hline $\mathrm{N}, 0,1.7310571591,0.1467716847,1.4546924046$ & $H, 0,0.1035975373,-0.9572013966,1.3716343556$ \\
\hline$H, 0,1.9152590766,1.0846096805,1.1011734315$ & $N, 0,-0.1049607895,-0.1718252628,-0.4733025337$ \\
\hline$H, 0,2.3001086746,-0.4677285401,0.8741616445$ & $H, 0,-0.9827609284,0.2451209665,-0.7653406995$ \\
\hline $\mathrm{N}, 0,2.3059350029,0.0948097884,2.7845966746$ & $\mathrm{H}, 0,0.6385841783,0.3754429603,-0.894353107$ \\
\hline \multirow{2}{*}{\multicolumn{2}{|c|}{$\begin{array}{l}H, 0,1.720066297,0.7065875691,3.3493379046 \\
H, 0,2.1045210825,-0.8441581267,3.1225590063\end{array}$}} \\
\hline & \\
\hline & $\mathrm{H} 2$ \\
\hline & $\begin{array}{c}H, 0,0 ., 0 .,-0.3707401718 \\
H, 0,0 ., 0 ., 0.3751980968\end{array}$ \\
\hline ( & $\left.\overbrace{\text { (iP) }}^{\mathrm{N}}\right|_{\mathrm{H}} ^{\mathrm{Fe}} \cdot \mathrm{CO}(\mathrm{iP})$ \\
\hline $\mathrm{H}$ & $H, 0,-1.0945767662,-0.5566094656,0$ \\
\hline$H, 0,0.2757896848,-1.3897297051,0$. & $\mathrm{Fe}, 0,0.0652980109,0.3869195364,0$. \\
\hline $\mathrm{Fe}, 0,-0.0313439637,0.14435987,0$ & $P, 0,0.216436033,0.1657597148,-2.1871402912$ \\
\hline $\mathrm{H}, 0,-0.1695446721,1.7084445624,0$ & $P, 0,0.216436033,0.1657597148,2.1871402912$ \\
\hline$P, 0,0.1778926137,0.1359242135,-2.1939206846$ & C,0,2.0279001424,0.4020339612,--2.4148226669 \\
\hline$P, 0,0.1778926137,0.1359242135,2.1939206846$ & C,0,2.0279001424,0.4020339612,2.4148226669 \\
\hline C,0,1.9890756572,0.4535049301,-2.4292633369 & H,0,2.1971955677,1.4823175786,-2.4115972574 \\
\hline C,0,1.9890756572,0.4535049301,2.4292633369 & $\mathrm{H}, 0,2.1971955677,1.4823175786,2.4115972574$ \\
\hline $\mathrm{H}, 0,2.1353027498,1.5363709441,-2.4747387049$ & $\mathrm{H}, 0,2.4148034671,-0.0051665163,-3.3506141669$ \\
\hline $\mathrm{H}, 0,2.1353027498,1.5363709441,2.4747387049$ & $\mathrm{H}, 0,2.4148034671,-0.0051665163,3.3506141669$ \\
\hline$H, 0,2.3766282005,0.0235274099,-3.35428644$ & C,0,2.6820980831,-0.2224605894,-1.1962096673 \\
\hline$H, 0,2.3766282005,0.0235274099,3.35428644$ & C,0,2.6820980831,-0.2224605894,1.1962096673 \\
\hline C,0,2.6968413461,-0.1111117499,-1.2172528689 & H,0,3.6982338613,0.179011588,-1.0737944394 \\
\hline C,0,2.6968413461,-0.1111117499,1.2172528689 & $\mathrm{H}, 0,3.6982338613,0.179011588,1.0737944394$ \\
\hline$H, 0,3.7625455247,0.1488752993,-1.2258866648$ & H,0,2.8010940974,-1.3071215053,-1.3505383308 \\
\hline$H, 0,3.7625455247,0.1488752993,1.2258866648$ & H,0,2.8010940974,-1.3071215053,1.3505383308 \\
\hline$H, 0,2.6131793653,-1.1999850923,-1.1931718081$ & $\mathrm{~N}, 0,1.8906814347,0.0449906331,0$. \\
\hline$H, 0,2.6131793653,-1.1999850923,1.1931718081$ & C,0,-0.5830199155,1.4228198834,3.3025592078 \\
\hline $\mathrm{N}, 0,2.0509483584,0.4015476781,0$ & C,0,-0.5830199155,1.4228198834,-3.3025592078 \\
\hline $\mathrm{H}, 0,2.1574251893,1.4128301101,0$ & $H, 0,-0.4120817565,2.341611607,2.7296603683$ \\
\hline$C, 0,-0.6339233344,1.5021796591,3.1774388608$ & $H, 0,-0.4120817565,2.341611607,-2.7296603683$ \\
\hline$C, 0,-0.6339233344,1.5021796591,-3.1774388608$ & C,0,-0.074060306,-1.5678387469,2.8199770875 \\
\hline$H, 0,-0.4764125269,2.3481704281,2.499268895$ & $C, 0,-0.074060306,-1.5678387469,-2.8199770875$ \\
\hline$H, 0,-0.4764125269,2.3481704281,-2.499268895$ & $H, 0,0.6486206406,-2.1230498405,2.2089423048$ \\
\hline$C, 0,-0.0677552038,-1.4992029069,3.0652716147$ & $H, 0,0.6486206406,-2.1230498405,-2.2089423048$ \\
\hline$C, 0,-0.0677552038,-1.4992029069,-3.0652716147$ & C,0,-1.1127776156,1.6367440523,0. \\
\hline$H, 0,0.7516592628,-2.0836176437,2.6292397128$ & $0,0,-1.8628775724,2.5292043757,0$ \\
\hline$H, 0,0.7516592628,-2.0836176437,-2.6292397128$ & $C, 0,0.2617464224,-1.7938011142,-4.2886931048$ \\
\hline$C, 0,-1.7305775057,-0.0594460541,0$ & $C, 0,0.2617464224,-1.7938011142,4.2886931048$ \\
\hline $0,0,-2.8882772408,-0.1898359709,0$ & $\mathrm{H}, 0,1.2362883249,-1.3894311895,-4.5672362782$ \\
\hline$C, 0,0.1000845636,-1.4866433669,-4.5785518776$ & $H, 0,1.2362883249,-1.3894311895,4.5672362782$ \\
\hline$C, 0,0.1000845636,-1.4866433669,4.5785518776$ & $H, 0,-0.4920949805,-1.3452528873,-4.938501931$ \\
\hline$H, 0,1.0121298091,-0.9790322451,-4.8983432772$ & H,0,-0.4920949805,-1.3452528873,4.938501931 \\
\hline$H, 0,1.0121298091,-0.9790322451,4.8983432772$ & $\mathrm{H}, 0,0.277431008,-2.8675518407,-4.5009313288$ \\
\hline$H, 0,-0.7481148191,-1.0032540659,-5.0671405273$ & $H, 0,0.277431008,-2.8675518407,4.5009313288$ \\
\hline$H, 0,-0.7481148191,-1.0032540659,5.0671405273$ & $C, 0,-1.4619976563,-2.1034811125,-2.4891727479$ \\
\hline$H, 0,0.1474082189,-2.5150824403,-4.9507481318$ & C,0,-1.4619976563,-2.1034811125,2.4891727479 \\
\hline$H, 0,0.1474082189,-2.5150824403,4.9507481318$ & $H, 0,-2.23083263,-1.6320088165,-3.1038949963$ \\
\hline$C, 0,-1.3732604136,-2.1720033192,-2.6587922395$ & $H, 0,-2.23083263,-1.6320088165,3.1038949963$ \\
\hline$C, 0,-1.3732604136,-2.1720033192,2.6587922395$ & $H, 0,-1.714632477,-1.9357893871,-1.4415775634$ \\
\hline$H, 0,-2.243367754,-1.6218819143,-3.0228318183$ & $\mathrm{H}, 0,-1.714632477,-1.9357893871,1.4415775634$ \\
\hline$H, 0,-2.243367754,-1.6218819143,3.0228318183$ & $H, 0,-1.4979159236,-3.1796800837,-2.6838848406$ \\
\hline$H, 0,-1.4511239385,-2.2550737698,-1.5745111353$ & $H, 0,-1.4979159236,-3.1796800837,2.6838848406$ \\
\hline$H, 0,-1.4511239385,-2.2550737698,1.5745111353$ & C,0,-2.087819641,1.2009115069,3.3992521174 \\
\hline$H, 0,-1.4179545248,-3.1801089042,-3.0826649013$ & C,0,-2.087819641,1.2009115069,-3.3992521174 \\
\hline$H, 0,-1.4179545248,-3.1801089042,3.0826649013$ & $H, 0,-2.3184793091,0.3611296895,4.0580838898$ \\
\hline
\end{tabular}


C,0,-2.1357349962,1.2783070717,3.3037522259 C,0,-2.1357349962,1.2783070717,-3.3037522259 $\mathrm{H}, 0,-2.3572650836,0.4465167621,3.9762002597$ $\mathrm{H}, 0,-2.3572650836,0.4465167621,-3.9762002597$ $\mathrm{H}, 0,-2.5992292825,1.0695783049,2.3388846294$ $\mathrm{H}, 0,-2.5992292825,1.0695783049,-2.3388846294$ $\mathrm{H}, 0,-2.6129010998,2.1718940038,3.7174136337$ $\mathrm{H}, 0,-2.6129010998,2.1718940038,-3.7174136337$ C, $0,-0.0090855998,1.8511032402,4.5233279636$ C, $0,-0.0090855998,1.8511032402,-4.5233279636$ $\mathrm{H}, 0,1.0722538777,1.985407525,4.4610448794$ $\mathrm{H}, 0,1.0722538777,1.985407525,-4.4610448794$ $\mathrm{H}, 0,-0.2132504569,1.0921900887,5.2781444556$ $\mathrm{H}, 0,-0.2132504569,1.0921900887,-5.2781444556$ $\mathrm{H}, 0,-0.4345098393,2.7934075842,4.8837630913$ $\mathrm{H}, 0,-0.4345098393,2.7934075842,-4.8837630913$

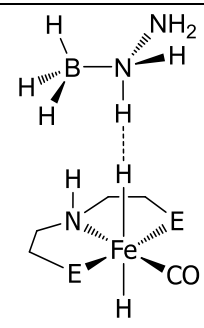

H, $0,-0.0946922065,1.6337107702,1.6468765944$ $\mathrm{N}, 0,0.0101819165,0.645814641,1.8746112629$ $\mathrm{Fe}, 0,0.021451774,-0.3688954544,0.0352832491$ $\mathrm{H}, 0,-0.0953845482,0.9841070169,-0.7503382947$ $\mathrm{H}, 0,0.2584663787,2.4067914951,-0.4861093916$ $\mathrm{N}, 0,0.1790913941,3.4506302619,-0.5290930916$ B, $0,-0.2313629698,4.0046706458,0.9035882895$ $\mathrm{H}, 0,0.1126649204,-1.6604022621,0.8971822209$ $P, 0,2.2123251441,-0.1664141278,0.1714485148$ $\mathrm{P}, 0,-2.1681527767,-0.4110294456,0.3032756349$ C, $0,2.4287638199,0.851023297,1.7022626605$ C, $0,-2.4128110321,0.4657261447,1.9162429584$ $\mathrm{H}, 0,2.3823454721,1.9051176876,1.4166454764$ $\mathrm{H}, 0,-2.5701675112,1.5255621296,1.7016660135$ $\mathrm{H}, 0,3.389588425,0.6785931876,2.1900742318$ $\mathrm{H}, 0,-3.2835029731,0.0997065584,2.4623385019$ C, $0,1.2733440542,0.5154314402,2.6180827576$ C, $0,-1.1423959116,0.2872234582,2.7166081913$ $\mathrm{H}, 0,1.2657895019,1.1698117809,3.497793541$ $\mathrm{H}, 0,-1.1587392704,0.9054006924,3.6222228417$ $\mathrm{H}, 0,1.3429231614,-0.518373777,2.9640918696$ $\mathrm{H}, 0,-1.0148978034,-0.7552120563,3.0163661094$ C, $0,-3.2031670578,0.5978913529,-0.8793915635$ C, $0,3.1014356356,0.8252167061,-1.1398683909$ $\mathrm{H}, 0,-2.5876304339,1.5032963175,-0.93207522$ $\mathrm{H}, 0,2.3640206437,1.613285424,-1.3256229462$ C, $0,-2.9636805055,-2.0715917935,0.6182634242$ C, $0,3.186102362,-1.7123749714,0.5610150018$ $\mathrm{H}, 0,-2.49510163,-2.3376903654,1.5738365807$ $\mathrm{H}, 0,2.7893162296,-1.9549392594,1.5545386528$ C, $0,0.0327935446,-1.23633496,-1.4438095343$ $0,0,0.0434757733,-1.8133252094,-2.4540929623$ C, $0,4.6949251453,-1.5418289303,0.6714352594$ C, $0,-4.4746103825,-2.0652348968,0.8069314871$ $\mathrm{H}, 0,4.9781496272,-0.7025351763,1.3094390499$ $\mathrm{H}, 0,-4.8126131161,-1.2872680377,1.4938906771$ $\mathrm{H}, 0,5.1496254193,-1.3939758899,-0.3100028554$ $\mathrm{H}, 0,-4.9911003101,-1.9273697927,-0.1447722218$ $\mathrm{H}, 0,5.1354151021,-2.4467685386,1.1017690253$ $\mathrm{H}, 0,-4.7960267387,-3.0287332085,1.2149342929$
$\mathrm{H}, 0,-2.3184793091,0.3611296895,-4.0580838898$ $\mathrm{H}, 0,-2.5361166887,1.0001452538,2.4242594849$ $\mathrm{H}, 0,-2.5361166887,1.0001452538,-2.4242594849$ H, $0,-2.5715037325,2.0894592593,3.8151562222$ $\mathrm{H}, 0,-2.5715037325,2.0894592593,-3.8151562222$ C, $0,0.0523133863,1.6024036706,4.6754085485$ C, $0,0.0523133863,1.6024036706,-4.6754085485$ $\mathrm{H}, 0,1.1295883652,1.7665807956,4.6133546388$ $\mathrm{H}, 0,1.1295883652,1.7665807956,-4.6133546388$ $\mathrm{H}, 0,-0.1234756661,0.7412788075,5.3206159065$ $\mathrm{H}, 0,-0.1234756661,0.7412788075,-5.3206159065$ $\mathrm{H}, 0,-0.3856048573,2.4769175492,5.1672678903$ H, $0,-0.3856048573,2.4769175492,-5.1672678903$

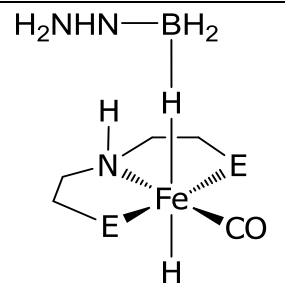

$\mathrm{H}, 0,0.846976103,-1.1388631307,0.0079764347$ Fe, $0,-0.0134916442,0.0983070934,0.0058220013$ $P, 0,-0.0642418013,-0.0996050499,2.2163230733$ $\mathrm{P}, 0,-0.0966157079,-0.1848614727,-2.1899470856$ C, $0,-1.7007288719,-0.9193342725,2.4665705965$ C, $0,-1.5979899522,-1.240636389,-2.3976194296$ $\mathrm{H}, 0,-2.4601299348,-0.1340358003,2.4543667493$ $\mathrm{H}, 0,-2.4402339595,-0.556474901,-2.5232914025$ $\mathrm{H}, 0,-1.7748877423,-1.4498352765,3.4172351114$ $\mathrm{H}, 0,-1.5355636278,-1.8912983395,-3.2713348218$ C, $0,-1.8985825012,-1.8530727783,1.2912842594$ C, $0,-1.7651551522,-2.033348975,-1.1193557199$ H, $0,-2.9113219645,-2.2748750428,1.2978053789$ $\mathrm{H}, 0,-2.733302903,-2.5503105143,-1.1098104978$ $\mathrm{H}, 0,-1.1948302576,-2.6883368819,1.341174703$ $\mathrm{H}, 0,-0.9828963787,-2.7910747901,-1.0244566235$ $\mathrm{N}, 0,-1.6626082826,-1.1280596428,0.034376985$ C, $0,-0.4376213131,1.3108887832,-3.2475444101$ C, $0,-0.1071458587,1.4140094133,3.305583241$ $\mathrm{H}, 0,-1.1524979527,1.8414782024,-2.6106858156$ $\mathrm{H}, 0,-0.6619073519,2.1114278544,2.6720243448$ C,0,1.257899809,-1.2254533255,-2.9402905596 C, $0,1.1390677647,-1.340891487,2.9240329215$ $\mathrm{H}, 0,1.065715592,-2.1907256076,-2.4559168576$ $\mathrm{H}, 0,0.8249429132,-2.2527011348,2.4027120435$ C, $0,1.3895229228,1.0971185903,-0.0028570356$ $0,0,2.345806588,1.7544534302,0.0056540619$ C, $0,1.0257681821,-1.5824181362,4.4234195599$ C, $0,1.1663711307,-1.4156846539,-4.4482861758$ $\mathrm{H}, 0,-0.0008197,-1.7722372417,4.7416364367$ $\mathrm{H}, 0,0.1675717577,-1.7111478339,-4.7748547153$ $\mathrm{H}, 0,1.4082664519,-0.7343966006,4.994095894$ $\mathrm{H}, 0,1.442785691,-0.5026654587,-4.9789908049$ $\mathrm{H}, 0,1.6228966896,-2.4573206028,4.6992284403$ H,0,1.8626472086,-2.1997955387,-4.7619120626 C, $0,2.579935366,-1.0592919832,2.5139788359$ C, $0,2.6500988813,-0.770858879,-2.5220250315$ $\mathrm{H}, 0,3.217888709,-1.895553487,2.8159692948$ $\mathrm{H}, 0,3.397662431,-1.4734471284,-2.9030061036$ $\mathrm{H}, 0,2.969986874,-0.1588631834,2.9906299186$ $\mathrm{H}, 0,2.8915607799,0.2163755967,-2.9203644852$ 
C,0,2.8234328697,-2.8639926474,-0.3684531834 C, $0,-2.5407874418,-3.1174760708,-0.4051382171$ $H, 0,3.1495229476,-2.6755907018,-1.393230282$ $\mathrm{H}, 0,-2.9091845801,-2.8778840254,-1.4048295033$ $\mathrm{H}, 0,1.7471364751,-3.036388679,-0.37873555$ $\mathrm{H}, 0,-1.4559944793,-3.2072039123,-0.4536183774$ $\mathrm{H}, 0,3.3128271119,-3.7820028311,-0.0288194872$ $\mathrm{H}, 0,-2.9500752723,-4.0935649596,-0.1267496762$ C, $0,-3.2333552815,-0.025506111,-2.2683525887$ C, $0,3.2632498583,0.0310511627,-2.4298583718$ $\mathrm{H}, 0,-3.8175266276,-0.9485618341,-2.2787364613$ $\mathrm{H}, 0,4.0035528771,-0.7635383828,-2.3149029935$ $\mathrm{H}, 0,-2.2299680155,-0.2545376549,-2.6302775233$ $\mathrm{H}, 0,2.3244833745,-0.4218028554,-2.7513128997$ $\mathrm{H}, 0,-3.697267756,0.6651086479,-2.979237757$ $\mathrm{H}, 0,3.6094686757,0.6893410256,-3.2323241032$ C,0,-4.5956005562,1.0068838664,-0.4145148523 C, $0,4.4078545007,1.4941316582,-0.7285982386$ $\mathrm{H}, 0,-4.5981147423,1.3957263857,0.6053272081$ $\mathrm{H}, 0,4.3144488303,2.057136087,0.2015848754$ $\mathrm{H}, 0,-5.3028491391,0.1796222881,-0.4643662807$ $\mathrm{H}, 0,5.2161372481,0.7733580924,-0.6085074331$ H, $0,-4.9745274838,1.7999451685,-1.0672994982$ $\mathrm{H}, 0,4.7122586307,2.1996657754,-1.5085353892$ $H, 0,1.0851628323,3.8217301699,-0.8053057463$ $\mathrm{N}, 0,-0.755799799,3.793973096,-1.5672810439$ $\mathrm{H}, \mathrm{O},-0.3350893978,5.2129084542,0.8366566093$ $\mathrm{H}, 0,-1.2916009787,3.4716075934,1.1833219308$ $\mathrm{H}, 0,0.6536641337,3.6672343269,1.6691661311$ $\mathrm{H}, 0,-0.7240558576,3.0486489711,-2.2601713755$ $\mathrm{H}, 0,-1.6748312243,3.7488790413,-1.1359248197$

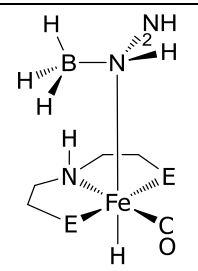

$\mathrm{H}, 0,-1.49929133,2.147374625,0.1145101856$ $\mathrm{N}, 0,-1.7908718499,1.169920424,0.042445141$ $\mathrm{Fe}, 0,0.0574898965,0.1998077172,0.0291574712$ $\mathrm{N}, 0,1.0715602691,2.0428584469,0.1635387766$ B, $0,0.2913626074,3.3962881877,0.2054545989$ $H, 0,1.0916310719,4.3227142534,0.3495115331$ $\mathrm{H}, 0,-0.7656194019,-1.0762517375,0.0109014136$ $P, 0,-0.2593168102,0.0484396724,2.2347274733$ $P, 0,-0.2402219847,0.1240698658,-2.1895608854$ C, $0,-1.8305268486,0.9844627476,2.4880345763$ C, $0,-1.7119079498,1.2154411718,-2.4056497709$ $\mathrm{H}, 0,-1.5651700784,2.0273690713,2.6720345951$ $\mathrm{H}, 0,-1.3441359177,2.2427696116,-2.4532663027$ $\mathrm{H}, 0,-2.3993798356,0.6211659293,3.3454571568$ $\mathrm{H}, 0,-2.2625162945,1.0048688437,-3.3239478324$ C, $0,-2.6320501908,0.8615943122,1.2125819881$ C, $0,-2.586829791,1.0279777662,-1.1893381393$ $\mathrm{H}, 0,-3.5043374621,1.5252002683,1.2351261656$ $\mathrm{H}, 0,-3.4140545953,1.7470273234,-1.1860583791$ $\mathrm{H}, 0,-2.9940272472,-0.1618368576,1.0883006162$ $\mathrm{H}, 0,-3.0205586557,0.0250292603,-1.1818206369$ C, $0,1.001854581,0.8244268784,-3.3859637587$ C, $0,0.9270276383,0.9026616439,3.3953781997$ $\mathrm{H}, 0,1.3506867146,1.6862154788,-2.8077004638$ $\mathrm{H}, 0,1.0270331016,1.8795742473,2.9069838384$ C, $0,-0.8531818532,-1.5271798396,-2.8168811085$ C, $0,-0.6876838051,-1.6559281598,2.8629613162$
$\mathrm{H}, 0,2.6682191659,-0.9404923079,1.4338528167$ $\mathrm{H}, 0,2.7438468236,-0.7330886768,-1.4370468376$ C, $0,0.8013747093,2.1838307512,-3.3978625332$ C, $0,1.2835734495,1.9945897873,3.53297029$ $H, 0,1.5510912743,1.7096696026,-4.0353028389$ $\mathrm{H}, 0,1.8713300192,1.3747826959,4.2128637324$ $\mathrm{H}, 0,1.2627348051,2.4056892668,-2.434529806$ $\mathrm{H}, 0,1.841828312,2.1053823391,2.6026087267$ $H, 0,0.5287095963,3.1365257563,-3.8615207316$ $\mathrm{H}, 0,1.1953506175,2.9866243621,3.9857395533$ C, $0,-1.1145838852,1.0558797701,-4.588272584$ C, $0,-0.872879933,1.2635221053,4.6145245507$ $\mathrm{H}, 0,-2.0024695793,0.4294375001,-4.4895553867$ $\mathrm{H}, 0,-1.8908169538,0.9032241331,4.4574647092$ $\mathrm{H}, 0,-0.4427316573,0.5882512364,-5.3081759757$ $\mathrm{H}, 0,-0.3745884474,0.5856573609,5.3082266474$ $\mathrm{H}, 0,-1.4376711904,2.0120344487,-5.0125472281$ $\mathrm{H}, 0,-0.9448822667,2.240640267,5.1030675688$ $\mathrm{H}, 0,-1.0150797281,1.4246531875,-0.1537489838$ $\mathrm{N}, 0,-3.3354425011,1.1206965455,-0.2412979932$ B, $0,-2.15187403,1.9881367111,0.1310476646$ $\mathrm{N}, 0,-3.7427057478,1.2239242212,-1.6125211658$ $\mathrm{H}, 0,-4.1203089031,1.2955522231,0.3789858222$ $\mathrm{H}, 0,-2.1691814515,2.2186366506,1.3217465167$ $\mathrm{H}, 0,-2.0364323616,3.0113790568,-0.5350982979$ $\mathrm{H}, 0,-2.4184776236,-0.4044666776,-0.0548624072$ $\mathrm{H}, 0,-4.5049348979,0.573214697,-1.7722592226$ $\mathrm{H}, 0,-4.1028690027,2.1587611619,-1.7988650536$

H, $,-1.4493500146,2.1254857667,-0.1348991959$ $\mathrm{N}, 0,-1.7766092541,1.152996046,-0.0366087855$ $\mathrm{Fe}, 0,0.0694931278,0.1995706506,-0.0200749604$ $\mathrm{N}, 0,0.8988567303,2.1352588028,0.1196539297$ B, $0,2.4440476924,2.327582462,-0.0508529101$ $\mathrm{H}, 0,2.7213464187,3.5178238253,0.1558228189$ $\mathrm{H}, 0,-0.7025972147,-1.1071677314,-0.0399312827$ $\mathrm{P}, 0,-0.2165224434,0.0868049736,2.1831118685$ $\mathrm{P}, 0,-0.2110889187,0.0686374707,-2.2231190265$ C, $0,-1.6689890638,1.2106529277,2.4070085428$ C, $0,-1.7847273869,1.0124287725,-2.4769735069$ $\mathrm{H}, 0,-1.2904981319,2.2363999977,2.441198461$ $\mathrm{H}, 0,-1.5355199019,2.0641721028,-2.6310611623$ $\mathrm{H}, 0,-2.2119254232,1.0255138466,3.3352342264$ $\mathrm{H}, 0,-2.3370321678,0.6708936557,-3.3539264051$ C, $0,-2.5619729138,1.0240686118,1.1995971712$ C,0,-2.6052211407,0.8509680384,-1.2148394455 $\mathrm{H}, 0,-3.3846187267,1.7488661579,1.205953423$ $\mathrm{H}, 0,-3.4852437249,1.5052111726,-1.2382150459$ $\mathrm{H}, 0,-3.0035976961,0.0243961144,1.2055917482$ $\mathrm{H}, 0,-2.957187223,-0.178693306,-1.1156168523$ C,0,1.0090196152,0.8497977559,-3.4012992911 C, $0,1.0877606033,0.785528313,3.3218413052$ $\mathrm{H}, 0,1.3343267303,1.7285945908,-2.8357299613$ $\mathrm{H}, 0,1.4881716274,1.6159629352,2.7305605817$ C, $0,-0.6814166655,-1.6257715474,-2.858113136$ C, $0,-0.8331262923,-1.5405774894,2.8528631411$ 
$\mathrm{H}, 0,-1.7902852053,-1.6323753368,-2.2580775026$ $\mathrm{H}, 0,-1.6241173994,-1.8340784927,2.3203439742$ C, $0,1.4905553578,-0.7508178967,0.0050037812$ $0,0,2.4402884234,-1.419732802,-0.0204692185$ C, $0,-0.9699128012,-1.7594261978,4.3558182684$ C, $0,-1.171443074,-1.5789193614,-4.3052334461$ $\mathrm{H}, 0,-1.6271127635,-0.9680664547,4.7198671206$ $\mathrm{H}, 0,-1.7937270039,-0.7444224537,-4.633000337$ $\mathrm{H}, 0,-0.0458789669,-1.7303492165,4.9358391916$ $\mathrm{H}, 0,-0.2597590793,-1.5808200487,-4.9053155553$ $\mathrm{H}, 0,-1.4565556837,-2.7166150358,4.5667122097$ $\mathrm{H}, 0,-1.7131183526,-2.5029384308,-4.530808364$ C, $0,0.3096313991,-2.7180710529,2.4201973563$ C, $0,0.0509751647,-2.6840703554,-2.4094396405$ $\mathrm{H}, 0,1.2980236126,-2.5522030496,2.8531245632$ $\mathrm{H}, 0,1.0306047166,-2.6229757764,-2.8863951286$ $\mathrm{H}, 0,0.4107474553,-2.7426823589,1.3362224672$ $H, 0,0.1974317927,-2.7122230533,-1.3301686571$ $\mathrm{H}, 0,-0.0344835484,-3.7031746704,2.7495147456$ $\mathrm{H}, 0,-0.4055094642,-3.6313767117,-2.7126222647$ C,0,2.1909847507,-0.1099903804,-3.572861749 C, $0,2.2929143696,0.2282960247,3.3946522172$ $\mathrm{H}, 0,1.9288037925,-0.9804291251,-4.1786806994$ $\mathrm{H}, 0,2.2573486755,-0.7388969346,3.9008018593$ $\mathrm{H}, 0,2.5859164894,-0.4696193455,-2.6209596801$ $H, 0,2.6685660014,0.0642571589,2.3829664257$ $\mathrm{H}, 0,2.9990261806,0.4179901526,-4.088391841$ $\mathrm{H}, 0,3.018824489,0.8517129687,3.9245949731$ C, $0,0.4608288501,1.3203401385,-4.7212277622$ C, $0,0.432334296,1.1799639262,4.8105487308$ $\mathrm{H}, 0,-0.3849778279,1.9988936719,-4.5996450995$ $\mathrm{H}, 0,-0.5710915428,1.6088254777,4.8233370478$ $\mathrm{H}, 0,0.1527036851,0.5059544512,-5.377066896$ $\mathrm{H}, 0,0.4274728729,0.2804703725,5.424757237$ $H, 0,1.2495851116,1.8767843228,-5.2382711711$ $\mathrm{H}, 0,1.1024103464,1.9012706953,5.2886846766$ $\mathrm{H}, 0,-0.470796014,3.3833167303,1.1660041072$ $\mathrm{H}, 0,-0.3425038481,3.5630740831,-0.8296116238$ $\mathrm{H}, 0,1.5031778189,1.9464215019,1.0772833276$ $\mathrm{N}, 0,2.1557170618,2.1757150262,-0.7857055494$ $\mathrm{H}, 0,2.7374338859,2.9582846893,-0.4897058757$ $\mathrm{H}, 0,2.725494097,1.3374103984,-0.7340828871$

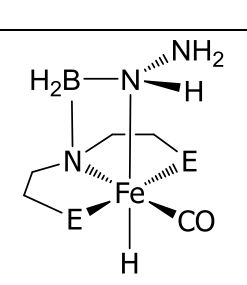

$\mathrm{N}, 0,0.0446022119,0.2138585871,-0.0251619323$ $\mathrm{Fe}, 0,2.1135120898,0.1681155602,0.0005118493$ $\mathrm{N}, 0,1.595094795,2.1399207281,0.097851517$ B, $0,0.0821353014,1.7724072566,0.2299477065$ $\mathrm{H}, 0,2.1223438846,-1.3523953863,0.0101222569$ $P, 0,1.8824971389,-0.1990720041,2.172131623$ $P, 0,1.9613304589,-0.0782629891,-2.2069197311$ C, $0,0.0528464816,-0.3865669259,2.3946600822$ C, $0,0.1960461054,0.3668627388,-2.4881745972$ $\mathrm{H}, 0,-0.3198707813,0.5267079799,2.8553897753$ $\mathrm{H}, 0,0.1566111227,1.4568787978,-2.5160051442$ $\mathrm{H}, 0,-0.1848354278,-1.2181128536,3.0610324026$ $\mathrm{H}, 0,-0.2044738774,-0.0207456345,-3.4265158571$ C, $0,-0.5761621437,-0.6007182763,1.0311085553$ C, $0,-0.581563899,-0.1604104686,-1.3024003238$ $\mathrm{H}, 0,-1.654469745,-0.3927845553,1.0840283819$
$\mathrm{H}, 0,-1.6261690918,-1.7838312637,-2.3243897215$ $\mathrm{H}, 0,-1.7842818337,-1.6358704269,2.3157123453$ C, $0,1.5405670702,-0.7039205823,-0.0177748691$ $0,0,2.4859858601,-1.3745744809,-0.0132676703$ C, $0,-1.1195971795,-1.5649052803,4.3482572779$ C, $0,-0.9561074855,-1.7063178321,-4.353874083$ $\mathrm{H}, 0,-1.7318158809,-0.721781544,4.673398208$ $\mathrm{H}, 0,-1.6268647964,-0.9191756809,-4.7023602749$ $\mathrm{H}, 0,-0.1959304033,-1.5603793532,4.9295507539$ $\mathrm{H}, 0,-0.0320192816,-1.6483123074,-4.9316154764$ $\mathrm{H}, 0,-1.6604154323,-2.4822060351,4.6008409752$ $\mathrm{H}, 0,-1.4266499474,-2.6667483592,-4.5861358561$ C, $0,0.0445021716,-2.7141720817,2.434483057$ C, $0,0.2794938051,-2.7214529591,-2.4156124907$ $H, 0,1.0218817535,-2.6843571998,2.9183090286$ $\mathrm{H}, 0,1.2665412555,-2.6048789706,-2.8654906849$ $\mathrm{H}, 0,0.1973296885,-2.7292447403,1.3554220725$ $\mathrm{H}, 0,0.3970798558,-2.7335461368,-1.3328370959$ $\mathrm{H}, 0,-0.4382881624,-3.6531075344,2.7218016441$ $\mathrm{H}, 0,-0.1114343201,-3.6960623687,-2.723648135$ C, $0,2.2409367425,-0.0292900693,-3.5778381277$ C, $0,2.2310467354,-0.200125509,3.530258777$ $\mathrm{H}, 0,2.0187386747,-0.9141042599,-4.1776191548$ $\mathrm{H}, 0,1.9275130064,-1.0328563847,4.1677254264$ $\mathrm{H}, 0,2.6471300269,-0.3547964896,-2.6192302435$ $\mathrm{H}, 0,2.5996463959,-0.6043748619,2.5870093761$ $\mathrm{H}, 0,3.0239830228,0.5335954221,-4.0938165811$ $\mathrm{H}, 0,3.0666761273,0.3060354956,4.0216958699$ C, $0,0.4562614256,1.3318938967,-4.7367429292$ C, $0,0.5878756342,1.3586664557,4.6424657551$ $\mathrm{H}, 0,-0.4177358885,1.9745057622,-4.6160105919$ $\mathrm{H}, 0,-0.2115067614,2.0881024469,4.5020004918$ $\mathrm{H}, 0,0.1813004926,0.5051609933,-5.3912545235$ $\mathrm{H}, 0,0.2243906309,0.5814536361,5.314608815$ $\mathrm{H}, 0,1.2240296205,1.9181291178,-5.251723178$ $\mathrm{H}, 0,1.4133510653,1.8710504291,5.1465674406$ $\mathrm{H}, 0,2.777143385,2.0193889681,-1.1871935846$ $\mathrm{H}, 0,3.0173633837,1.6219054023,0.7671929424$ $\mathrm{N}, 0,0.1350262727,3.1290613038,-0.6207668129$ $\mathrm{H}, 0,0.6829393384,2.37665686477,1.0811230462$ $\mathrm{H}, 0,0.5636570217,4.0366484678,-0.4347148938$ $\mathrm{H}, 0,0.3304702612,2.9544653331,-1.6021605744$<smiles>NNCN1CC[PH](P)C1(F)C=O</smiles>

$\mathrm{H}, 0,1.4240679267,0.0127579451,0.0212890959$ $\mathrm{Fe}, 0,-0.0628078185,0.0664146252,-0.0071714798$ $P, 0,-0.0899018322,-0.2183246295,2.1792824761$ $\mathrm{P}, 0,-0.0135137848,-0.186965353,-2.1987332359$ C, $0,-1.6938729482,-1.096611523,2.3817137717$ C, $0,-1.5460205567,-1.1715120697,-2.4713294893$ $\mathrm{H}, 0,-2.4678463738,-0.3258693495,2.3871529531$ H, $0,-2.3756297845,-0.4644191732,-2.5472754695$ $\mathrm{H}, 0,-1.7683427743,-1.6636296588,3.3111037402$ $\mathrm{H}, 0,-1.5205338579,-1.7650241631,-3.3868382789$ C, $0,-1.8455204064,-1.9786297685,1.1564732293$ C,0,-1.7294322307,-2.0320799634,-1.2353286207 $\mathrm{H}, 0,-2.9032427115,-2.2464444633,1.0181952309$ $\mathrm{H}, 0,-2.7784604371,-2.3525802611,-1.1624661555$ $\mathrm{H}, 0,-1.3095844115,-2.9286083458,1.3063624103$ 
$\mathrm{H}, 0,-1.6143954519,0.21310792,-1.3323283374$ $\mathrm{H}, 0,-0.4622211377,-1.6511265353,0.7436757274$ $\mathrm{H}, 0,-0.6319882457,-1.2537132582,-1.3551062327$ C,0,2.9191135248,0.9815792951,-3.4112608711 C, $0,2.3044543184,1.1779470318,3.3638420624$ $\mathrm{H}, 0,2.9703230209,1.9223286094,-2.8600515701$ $H, 0,1.7803006223,2.0160645269,2.8882131347$ C, $0,2.0528506973,-1.8586832123,-2.771012813$ C, $0,2.5579730244,-1.8280481953,2.7834374194$ $\mathrm{H}, 0,1.2457495894,-2.3015520288,-2.1763211912$ $H, 0,1.9399594053,-2.5222676564,2.2015445759$ C,0,3.8314447876,0.2727358381,-0.0102378867 $0,0,4.9910637662,0.3567230769,-0.045636658$ C,0,2.3452120139,-2.1186096945,4.2628856119 C,0,1.7509894607,-2.0956569425,-4.2450560077 $\mathrm{H}, 0,1.3327678069,-1.8858936221,4.597771736$ $\mathrm{H}, 0,0.8322300722,-1.6044162722,-4.5703991063$ $H, 0,3.0470945487,-1.5571754929,4.8817802491$ $H, 0,2.5674076316,-1.7469381305,-4.8801203532$ $H, 0,2.5209230561,-3.1818376053,4.4537430887$ $\mathrm{H}, 0,1.6322224777,-3.1686290223,-4.4267837378$ C, $0,4.0083630693,-2.0554519395,2.3805424826$ C,0,3.3483237844,-2.5471206574,-2.3564873304 $\mathrm{H}, 0,4.6804024119,-1.3413574374,2.8607996624$ $\mathrm{H}, 0,4.2006911313,-2.1925076935,-2.9373948869$ $\mathrm{H}, 0,4.1379634139,-1.9737649484,1.3021909055$ $H, 0,3.560324585,-2.3849011356,-1.2991983643$ $H, 0,4.3213769368,-3.0592621797,2.6840116623$ $\mathrm{H}, 0,3.2619402326,-3.625351459,-2.5231441497$ C, $0,4.3383235407,0.4719439812,-3.6313497659$ C,0,3.7957216753,1.4875669897,3.3547354602 $\mathrm{H}, 0,4.3496522909,-0.4194029032,-4.2615738366$ $\mathrm{H}, 0,4.368162803,0.6936292279,3.8395195833$ $H, 0,4.843853449,0.2322748603,-2.6949314163$ $\mathrm{H}, 0,4.1806124937,1.6099638772,2.3410823683$ $H, 0,4.9289375527,1.2396041509,-4.1407906768$ $\mathrm{H}, 0,3.990501531,2.4143338592,3.9028234981$ C,0,2.2173982857,1.2671061502,-4.7339780118 C,0,1.7647411244,1.0674893275,4.7853272523 $\mathrm{H}, 0,1.2291543354,1.7052488804,-4.5869732707$ $\mathrm{H}, 0,0.7177149287,0.7609878117,4.8133043976$ $H, 0,2.1063379129,0.3712467888,-5.345637132$ $\mathrm{H}, 0,2.3408842557,0.3615075046,5.3826139114$ $H, 0,2.8110915216,1.9858134449,-5.3086235619$ $\mathrm{H}, 0,1.8335571199,2.0441091063,5.2750475107$ $\mathrm{H}, 0,-0.5953337321,2.3755168333,-0.5875788126$ $\mathrm{H}, 0,-0.2963760769,2.0287449317,1.3582822533$ $\mathrm{H}, 0,1.9452502671,2.5619944972,0.9504403845$ $\mathrm{N}, 0,1.9270233663,3.0025314164,-1.002816862$ $\mathrm{H}, 0,1.5373007247,3.9256343971,-0.8195485806$ $\mathrm{H}, 0,2.93748756,3.1082744329,-1.0124039784$
$\mathrm{H}, 0,-1.1344171368,-2.9537200285,-1.323174963$ $\mathrm{N}, 0,-1.3429932354,-1.2946622125,-0.0333186278$ C, $0,-0.1995952257,1.311236376,-3.2907461633$ C,0,-0.1792027477,1.2564449987,3.3079145558 $\mathrm{H}, 0,-0.8873879994,1.9229020188,-2.6945802652$ $\mathrm{H}, 0,-0.8207788301,1.9242867858,2.7232945615$ C,0,1.3344124113,-1.3062306621,-2.845688933 C,0,1.1527793086,-1.4641299191,2.806987827 $\mathrm{H}, 0,1.1062427855,-2.2291203607,-2.2976493307$ $\mathrm{H}, 0,0.8763629819,-2.3411822848,2.2085451103$ C, $0,0.1794684683,1.7661907519,0.0155437695$ $0,0,0.2947364334,2.9245433944,0.0335815588$ C, $0,1.0155552393,-1.8246995238,4.280856334$ C,0,1.2661001387,-1.6009512122,-4.3388086853 $\mathrm{H}, 0,-0.0117728691,-2.0596038237,4.5642359528$ $\mathrm{H}, 0,0.266049633,-1.8878486511,-4.6680524249$ $\mathrm{H}, 0,1.3686890372,-1.0128082081,4.9194804014$ $H, 0,1.5831390657,-0.7353592073,-4.9239138344$ $H, 0,1.6282610824,-2.7042960697,4.5022615016$ H,0,1.9434727919,-2.4254844296,-4.5821342883 C,0,2.5939730044,-1.1094038149,2.4621488562 C,0,2.7347798163,-0.8624117317,-2.4403795849 $\mathrm{H}, 0,3.2450909191,-1.9611629485,2.6812775139$ $\mathrm{H}, 0,3.4533467347,-1.6541999725,-2.6728198189$ $\mathrm{H}, 0,2.951394636,-0.2617765071,3.0492080661$ $\mathrm{H}, 0,3.0446983614,0.033130528,-2.9816604491$ $\mathrm{H}, 0,2.7004041359,-0.8591148768,1.4061213472$ $\mathrm{H}, 0,2.7922095166,-0.6490846757,-1.3724717341$ C,0,1.1163009292,2.0700679439,-3.4128302303 C,0,1.1839850306,1.9206254343,3.459604202 $\mathrm{H}, 0,1.8034958951,1.5528915712,-4.0856014565$ $\mathrm{H}, 0,1.8295907324,1.3484178022,4.1294085828$ $\mathrm{H}, 0,1.6120088156,2.1908650002,-2.4478222276$ $\mathrm{H}, 0,1.6974217134,2.0261550727,2.501745893$ $\mathrm{H}, 0,0.9369086183,3.0670314349,-3.8252696863$ $\mathrm{H}, 0,1.0655628893,2.9198494694,3.8883207781$ C,0,-0.8459010742,1.0742074327,-4.6496736537 C, $0,-0.8564227446,1.0265789429,4.6524346397$ $\mathrm{H}, 0,-1.7985288932,0.5479865924,-4.5668871904$ $\mathrm{H}, 0,-1.8440454415,0.5759369557,4.5410347182$ $\mathrm{H}, 0,-0.1982802702,0.5023947141,-5.3144809025$ $\mathrm{H}, 0,-0.2618227694,0.3901323876,5.3083865054$ $\mathrm{H}, 0,-1.0432678405,2.0376320795,-5.1304991068$ $\mathrm{H}, 0,-0.9910612153,1.9880286172,5.158085915$ $\mathrm{N}, 0,-3.2901139776,1.0556750432,-0.3136018207$ $\mathrm{N}, 0,-4.319267012,0.3886646534,-1.0817509296$ B, $0,-3.7584841117,1.7864500883,1.0152061516$ $\mathrm{H}, 0,-4.6697776646,-0.3472915803,-0.4732058473$ $\mathrm{H}, 0,-5.0828963787,1.0584730692,-1.1496696464$ $\mathrm{H}, 0,-4.2664557156,0.9401613916,1.7296388664$ $\mathrm{H}, 0,-2.7655105021,2.2714549552,1.5223943725$ $\mathrm{H}, 0,-4.5640557988,2.641070476,0.6866641419$ $\mathrm{H}, 0,-2.5494449853,0.346152557,-0.1324279191$ $\mathrm{H}, 0,-2.8440849845,1.7163779136,-0.9476829803$ 


\section{Solid-state NMR experiments}

Solid-state MAS NMR studies were conducted on a Bruker Avance III $300\left(v_{\mathrm{L}}\left({ }^{1} \mathrm{H}\right)=300.1 \mathrm{MHz}, 7.05 \mathrm{~T}\right)$ and a Bruker DSX $500\left(v_{\mathrm{L}}\left({ }^{1} \mathrm{H}\right)=500.2 \mathrm{MHz}, 11.74 \mathrm{~T}\right)$ spectrometer.

All ${ }^{11}$ B MAS NMR spectra were measured at $11.7 \mathrm{~T}(160.5 \mathrm{MHz})$ with a MAS frequency of $25 \mathrm{kHz}$. To achieve uniform excitation, a short pulse in the range of 0.5-0.75 $\mu$ s and a relaxation delay of $2 \mathrm{~s}$ was used. High-power ${ }^{1} \mathrm{H}$ decoupling was applied during acquisition $\left(\mathrm{SW}_{\mathrm{F}}-\mathrm{TPPM} 15, v_{\mathrm{RF}}=142 \mathrm{kHz}\right)$. The $1 \mathrm{D}$ and $2 \mathrm{D}{ }^{11} \mathrm{~B}$ MAS NMR spectra were referenced using $\mathrm{BF}_{3} \cdot \mathrm{OEt}_{2}(\delta=0.0 \mathrm{ppm})$.

The $2 \mathrm{D}{ }^{11} \mathrm{~B}$ MQMAS NMR spectra were measured at $11.7 \mathrm{~T}(160.5 \mathrm{MHz})$ with a spinning speed of $25 \mathrm{kHz}$ and the z-filtered MQMAS sequence. ${ }^{18}$ For excitation and conversion of triple-quantum coherences short pulses of $5 \mu \mathrm{s}$ and $2 \mu$ s were used, respectively, followed by a soft central-transition read-out pulse of $10 \mu \mathrm{s}$. High-power ${ }^{1} \mathrm{H}$ decoupling was conducted during the whole pulse sequence and acquisition (SWFTPPM15, $v_{\mathrm{RF}}=$ $142 \mathrm{kHz}$ ). Shearing of the data was performed within data processing.

The ${ }^{15} \mathrm{~N}\left\{{ }^{1} \mathrm{H}\right\}$ cross-polarization (CP)/MAS NMR spectra were acquired at 7.1 T (30.4 MHz) using a $5.8 \mu \mathrm{s}$ $\pi / 2$ pulse for ${ }^{1} \mathrm{H}\left(v_{\mathrm{RF}}=43 \mathrm{kHz}\right)$ for excitation followed by a ramped $5.0 \mathrm{~ms}$ spinlock pulse on ${ }^{1} \mathrm{H}$ from 70 to $100 \%$ to a maximum nutation frequency of $50 \mathrm{kHz}$. The spin lock pulse on ${ }^{15} \mathrm{~N}$ had used $v_{\mathrm{RF}}=43 \mathrm{kHz}$. A recycle delay of $1.5 \mathrm{~s}$ was employed and ${ }^{1} \mathrm{H}$ decoupling was applied during acquisition $\left(\mathrm{SW}_{\mathrm{F}}\right.$-TPPM15, $v_{\mathrm{RF}}$ $=63 \mathrm{kHz}$ ).

Spectral deconvolution was performed with DMFIT software (version 2011). ${ }^{19}$

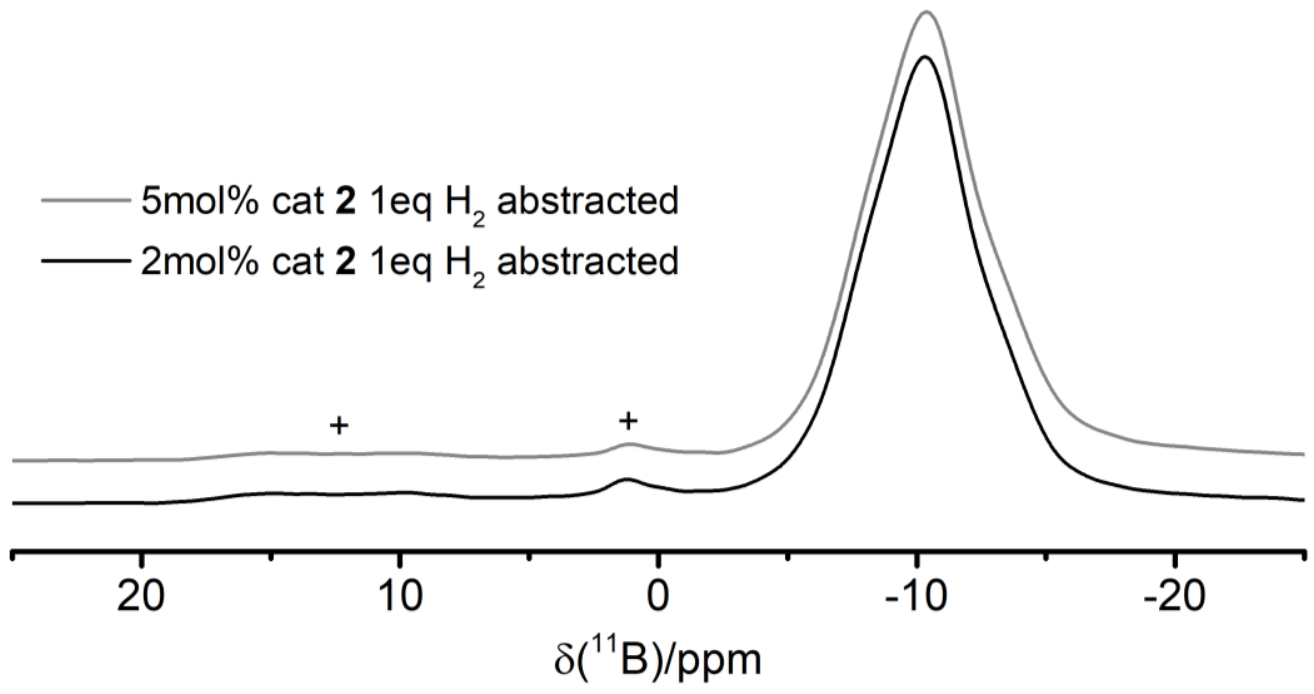

Figure S23. ${ }^{11}$ B MAS NMR spectra of $\mathbf{H B}$ dehydrogenated using 2 at a concentration of 2 mol\% (black) and $5 \mathrm{~mol} \%$ (grey) corresponding to an abstraction of up to one equivalent of $\mathrm{H}_{2}$. 
Table S4. Isotropic ${ }^{11} \mathrm{~B}$ chemical shifts and quadrupolar coupling parameters for the boron species in $\mathbf{H B}$ dehydrogenated with $2 \mathrm{~mol} \%$ of $\mathbf{1 - B \mathbf { H } _ { 3 }}$ up to an abstraction of 1 eq. $\mathrm{H}_{2}$ determined from analysis of the $2 \mathrm{D}{ }^{11} \mathrm{~B}$ MQMAS NMR (Figure 5) and the ${ }^{11} \mathrm{~B}$ MAS NMR spectrum (Figure 4, black line). Values in brackets are calculated using formula $S 1 . \eta_{\mathrm{Q}}$ is determined from the deconvolution of the ${ }^{11} \mathrm{~B}$ MAS NMR spectrum in Figure 4. The SOQE parameter is determined form of the MQMAS NMR spectrum.

\begin{tabular}{c|c|c|c} 
& Species & $\mathrm{A}$ & $\mathrm{B}$ \\
\hline \multirow{2}{*}{${ }^{11} \mathrm{~B}$ MQMAS } & $\mathrm{F} 2 / \mathrm{ppm}$ & -10.3 & -6.7 \\
& $\mathrm{~F} 1 / \mathrm{ppm}$ & -5.4 & -2.2 \\
& $\delta_{\text {iso }} / \mathrm{ppm}$ & -7.2 & -3.8 \\
& $\mathrm{SOQE} / \mathrm{MHz}$ & $1.78(1.65)$ & $1.71(1.55)$ \\
\hline \hline 1D spectral & $\delta_{\text {iso }} / \mathrm{ppm}$ & -7.3 & -4.0 \\
deconvolution & $\eta_{\mathrm{Q}}$ & 0.7 & 0.8 \\
& $\mathrm{C}_{\mathrm{Q}} / \mathrm{MHz}$ & 1.62 & 1.53 \\
& & & \\
& & & \\
\multicolumn{3}{c}{$S O Q E=C_{q} \sqrt{1+\frac{\eta^{2}}{3}}$} &
\end{tabular}

Table S5. Isotropic ${ }^{11} \mathrm{~B}$ chemical shifts and quadrupolar coupling parameters for the boron species in $\mathbf{H B}$ dehydrogenated with 2 mol\% of 2 up to an abstraction of 1 eq. $\mathrm{H}_{2}$ determined from analysis of the $2 \mathrm{D}{ }^{11} \mathrm{~B}$ MQMAS NMR (Figure S21) and the ${ }^{11}$ B-MAS spectrum (Figure 4, grey line). Values in brackets are calculated using formula $\mathrm{S} 1 . \eta_{\mathrm{Q}}$ is determined from the deconvolution of the ${ }^{11} \mathrm{~B}$ MAS NMR spectrum in Figure 4. The SOQE parameter is determined form of the MQMAS NMR spectrum.

\begin{tabular}{c|c|c|c} 
& Species & $\mathrm{A}$ & $\mathrm{B}$ \\
\hline \multirow{3}{*}{${ }^{11} \mathrm{~B}$ MQMAS } & $\mathrm{F} 2 / \mathrm{ppm}$ & -10.3 & -6.7 \\
& $\mathrm{~F} 1 / \mathrm{ppm}$ & -5.4 & -2.2 \\
& $\delta_{\text {iso }} / \mathrm{ppm}$ & -7.2 & -3.8 \\
& $\mathrm{SOQE} / \mathrm{MHz}$ & $1.78(1.65)$ & $1.71(1.55)$ \\
\hline \hline \multirow{2}{*}{ 1D spectral } & $\delta_{\text {iso }} / \mathrm{ppm}$ & -7.3 & -4.3 \\
deconvolution & $\eta_{\mathrm{Q}}$ & 0.7 & 0.8 \\
& $\mathrm{C}_{\mathrm{Q}} / \mathrm{MHz}$ & 1.63 & 1.53 \\
& & &
\end{tabular}




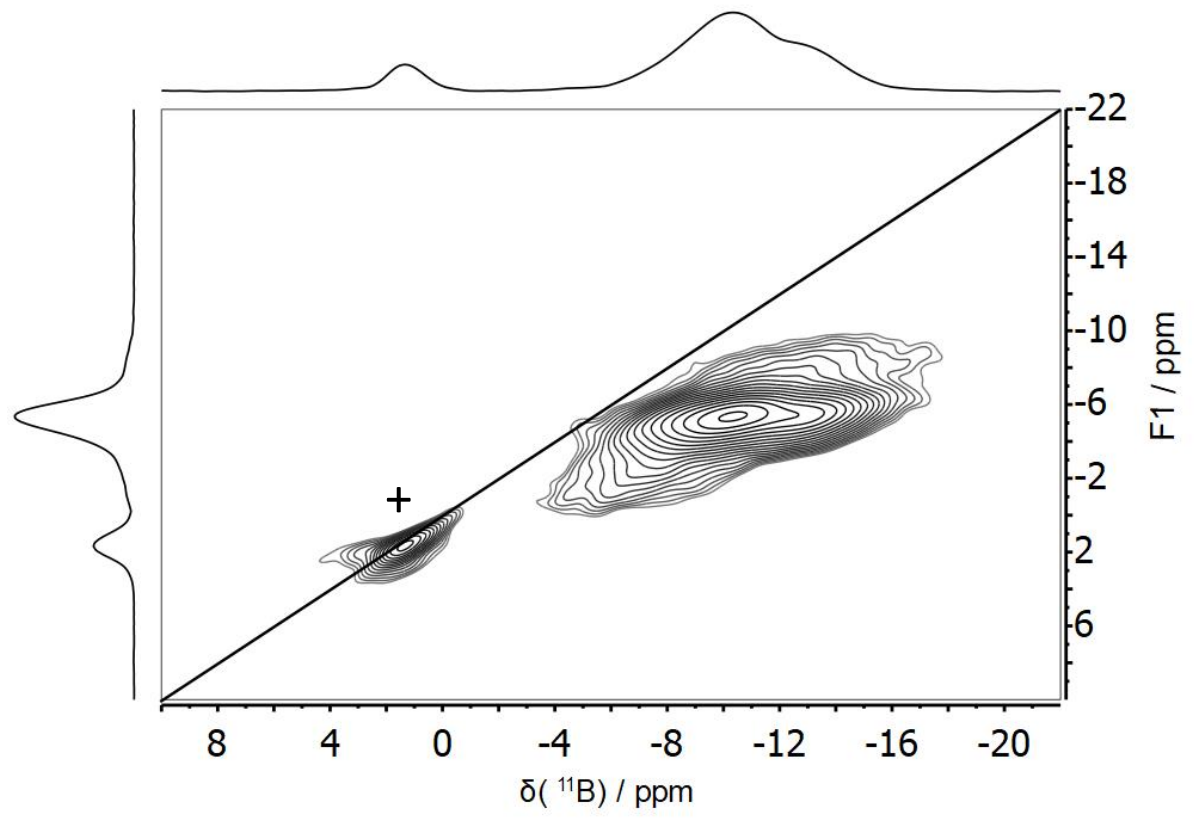

Figure S24. 2D ${ }^{11} \mathrm{~B}$ MQMAS NMR spectrum of $\mathbf{H B}$ dehydrogenated up to an abstraction of 1.0 eq. $\mathrm{H}_{2}$ using $2 \mathrm{~mol} \%$ of catalyst 2 , and measured at a magnetic-field strength of $11.7 \mathrm{~T}$ and a MAS frequency of $25 \mathrm{kHz}$. An impurity caused by reaction of the white precipitate with air is marked with a + sign. Table S6 summarizes the determined ${ }^{11} \mathrm{~B}$ chemical shifts and quadrupolar coupling parameters.

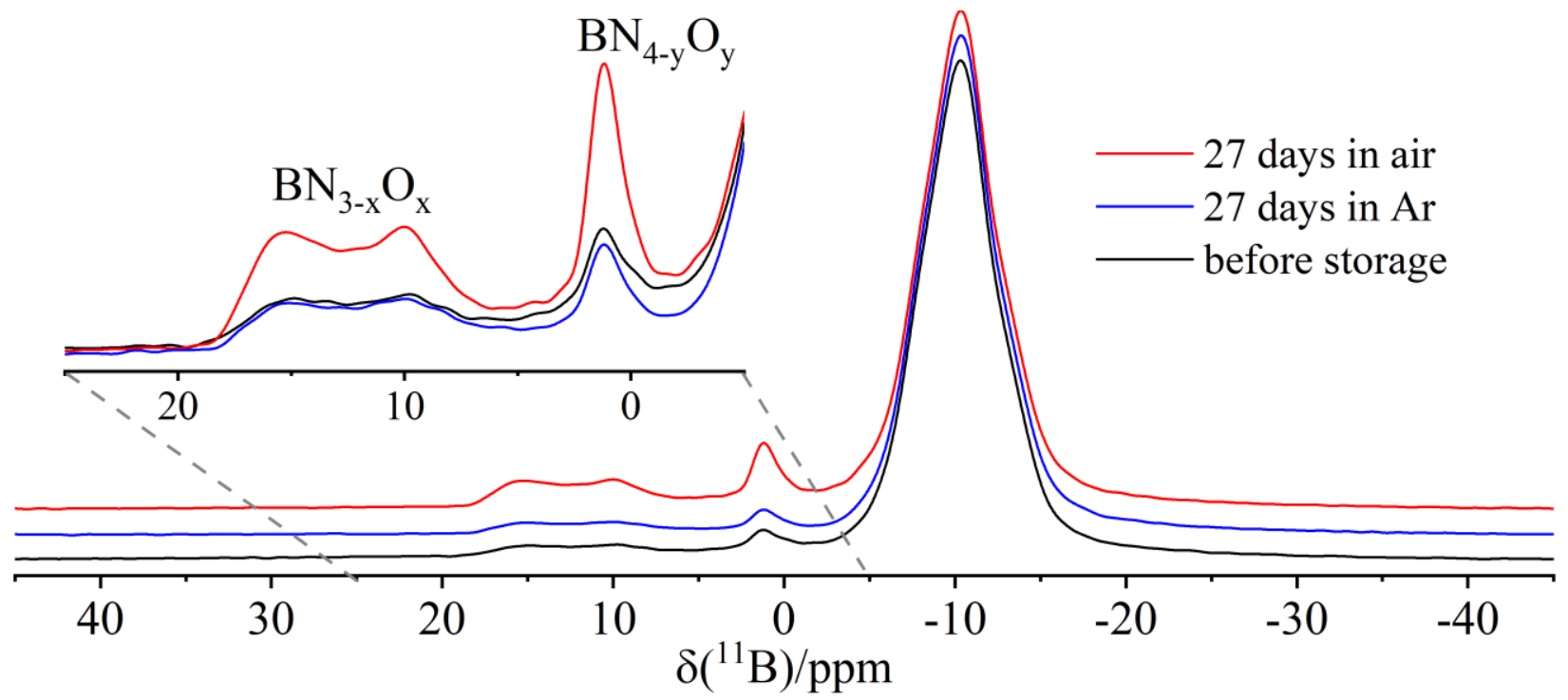

Figure S25. ${ }^{11}$ B MAS NMR spectra of HB dehydrogenated up to an abstraction of 1.0 eq. $\mathrm{H}_{2}$ using $2 \mathrm{~mol} \%$ of catalyst 2 . Depicted are the ${ }^{11}$ B MAS NMR spectra for three different fractions of the same sample. The first one was taken before storage of the powder (black line), while the other two samples were stored under argon (blue line) and in air (red line) for 27 days. Note that changes in the ${ }^{11}$ B MAS NMR spectra are only visible for the sample that was in air contact, showing increased signal intensity of boron species centered at 13 and $1 \mathrm{ppm}$. 


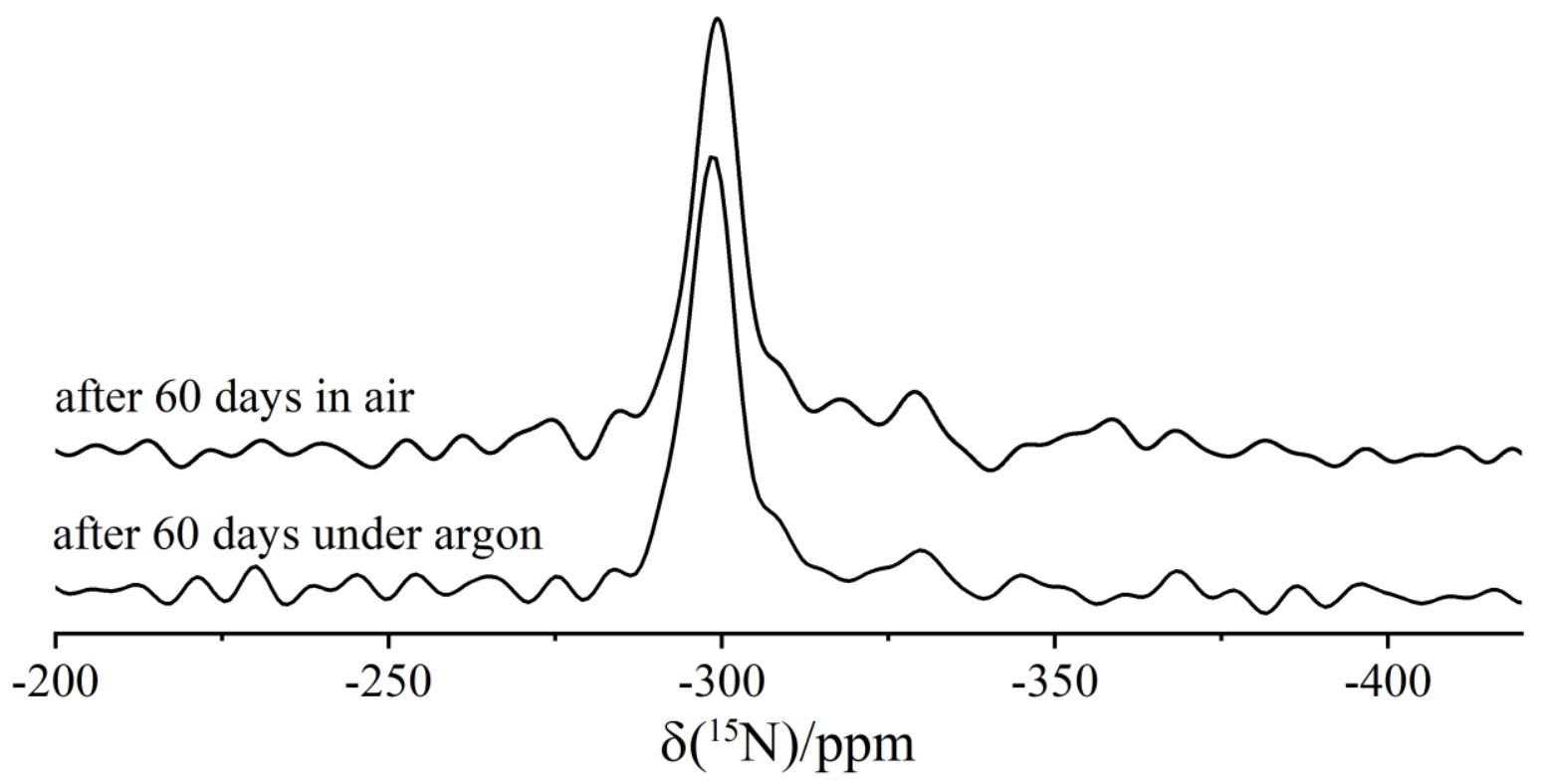

Figure S26. ${ }^{15} \mathrm{~N}\left\{{ }^{1} \mathrm{H}\right\}$ CP/MAS NMR spectra for $\mathbf{H B}$ dehydrogenated releasing 1.0 eq. $\mathrm{H}_{2}$ using 2 mol\% of catalyst 2. Depicted are the two ${ }^{15} \mathrm{~N}\left\{{ }^{1} \mathrm{H}\right\}$ CP/MAS NMR spectra for the same dehydrogenated sample stored in air (top) and under argon (bottom) for 60 days. No significant changes due to conversion of the dehydrogenated material in air can be observed.

DFT calculations: DFT calculations of NMR parameters were conducted starting from molecular structures created in $\mathrm{Jmol}^{20}$ followed by geometry optimization as described in the following. For polymeric structures, we used a chain of five monomers and evaluated the NMR parameters of the monomer in the middle of this chain. Geometry optimization were performed using the TPSS functional ${ }^{21}$ and NMR chemical shift calculations employed the B3LYP ${ }^{22,23}$ functional using TURBOMOLE (version $6.5)^{24,25}$ and a def2-TZVP basis set. ${ }^{26}$ The ${ }^{11} \mathrm{~B}$ quadrupolar coupling parameters were calculated at the GGA DFT level using GAUSSIAN (version GAUSSIAN09) ${ }^{27}$ and the B97-D functional. ${ }^{28}$ These calculations employed the def2-TZVP basis set obtained from the EMSL database ${ }^{29,30}$ and further included selected functions from the cc-pCVTZ basis set ${ }^{31,32}$ for boron to obtain the best description of the atomic wave functions at the nuclear site. As small distortions in the molecular geometry lead to minor deviations of NMR parameters for chemically equivalent nuclei, we chose the average value of the respective boron atoms.

Instead of referencing to only a single calculated compound, we have referred our ${ }^{11} \mathrm{~B}$ chemical shift calculations using the literature known isotropic ${ }^{11} \mathrm{~B}$ chemical shifts of four different reference structures (struct. 1-4, Figure 6). We calculated each of these structures, plotted the experimental shift known from literature $^{3,33,34}$ against the calculated chemical shielding (Figure S27) and applied a linear regression. Note that the slope of the resulting line is close to unity as expected. However, using this equation to calculate shifts of the discussed reference compounds, deviations of up to $1.7 \mathrm{ppm}$ (for $\mathrm{HB}$, see Figure 6) were obtained, which have to be kept in mind evaluating the computed structures and their DFT parameters. The experimental ${ }^{11} \mathrm{~B}$ isotropic chemical shifts of the precipitates obtained from HB dehydrogenation are in between those of the reference compounds. 


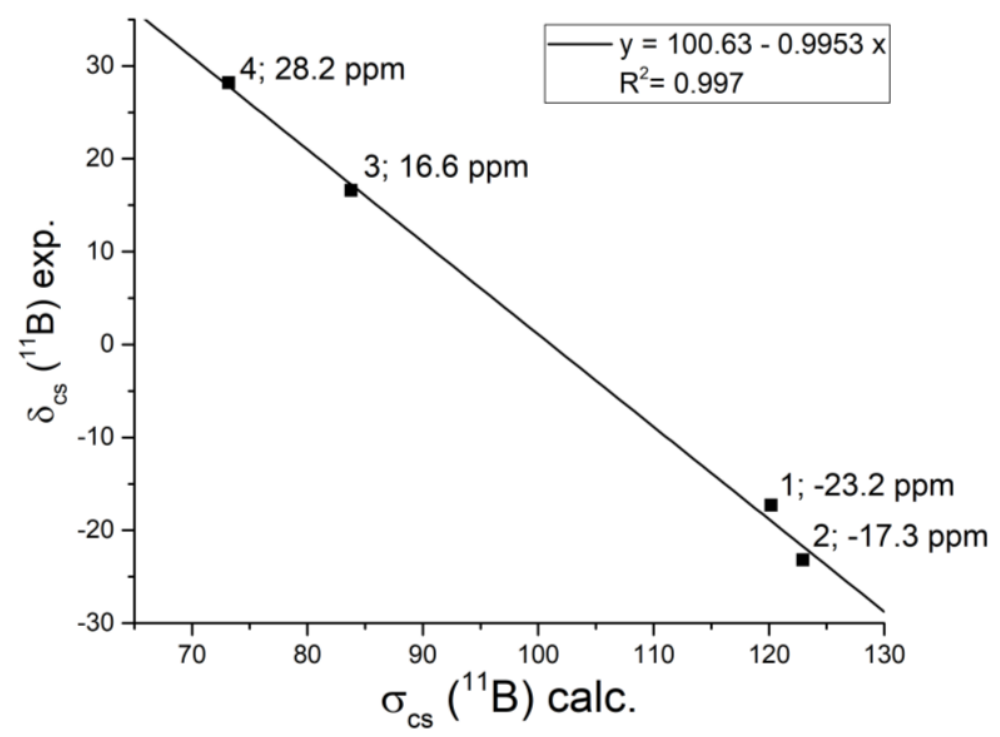

Figure S27. The experimentally determined ${ }^{11} \mathrm{~B}$ isotropic chemical shifts ${ }^{3,33,34}$ of reference structures 1-4 (Figure 7) plotted against calculated chemical shielding. Moreover, the function resulting from linear regression is depicted.

For referencing of ${ }^{15} \mathrm{~N}$ chemical shifts we utilized the same procedure as for the ${ }^{11} \mathrm{~B}$ chemical shifts described above, which included literature known ${ }^{15} \mathrm{~N}$ chemical shifts ${ }^{35,36,37}$ of five reference compounds (struct. N1-N4, and 2, see Figure S29). The linear regression depicted in Figure S28 results in a slope that differs from unity despite the good correlation coefficient $\mathrm{R}^{2}$ for the very broad chemical shift range of $\sim 360 \mathrm{ppm}$. This shows that it is particularly important for ${ }^{15} \mathrm{~N}$ chemical shifts to use several reference compounds to take this effect into account.

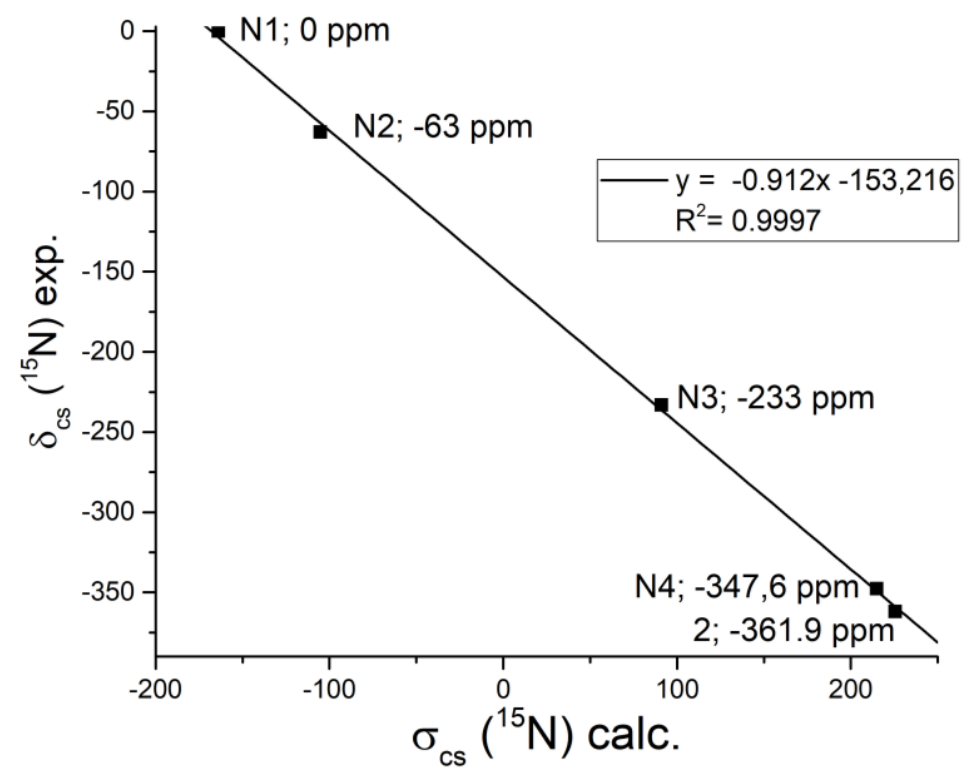

Figure S28. The experimentally determined ${ }^{15} \mathrm{~N}$ isotropic chemical shifts ${ }^{35,36,37}$ of reference structures N1-N4 and 2 (see Figure S29) plotted against calculated chemical shielding. The function resulting from linear regression is depicted additionally. 


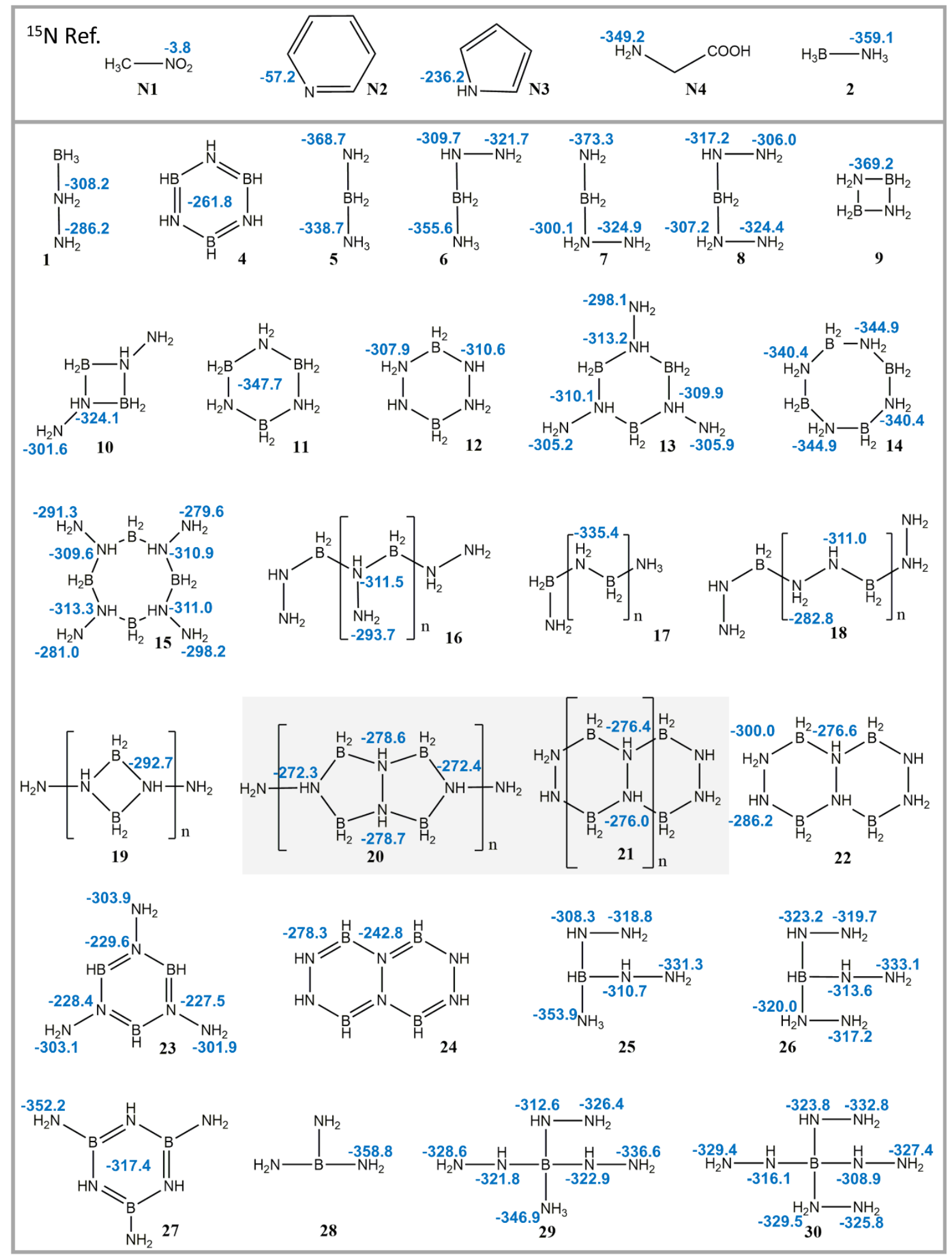

Figure S29. Summary of the $\mathrm{B}_{\mathrm{x}} \mathrm{N}_{\mathrm{y}} \mathrm{H}_{\mathrm{z}}$ structures used for DFT geometry optimization and calculation of isotropic ${ }^{15} \mathrm{~N}$ chemical shifts assigned to the respective nitrogen species given in blue. 


\section{XPS analysis}

The powder samples were attached to a conductive carbon tape and the resulting homogenous layer slightly pressed using a spatula. The sample was stored in the ultra-high vacuum chamber of the XPS for longer than $12 \mathrm{~h}$ to completely remove any volatile species before the actual measurements. XPS (Axis Ultra DLD, Kratos, U.K.) was measured using a monochromatic $\mathrm{Al} \mathrm{K}$ source $(\mathrm{h} v=1486.6 \mathrm{eV})$ at a 10 $\mathrm{mA}$ emission current and a $12 \mathrm{kV}$ accelerating voltage source power. To compensate for the charging of the sample, the charge neutralizer was used. The measurement was carried out at a $0^{\circ}$ angle of emission and with a pass energy of $40 \mathrm{eV}$ for core spectra (using the apparatus internal setting FOV2). The analysis area was approximately $100 \mu \mathrm{m} \times 100 \mu \mathrm{m}$. Sputter depth profiling was carried out using an Argon ion gun. The operating conditions were chosen to be a $3.8 \mathrm{kV}$ accelerating voltage and a $10 \mathrm{~mA}$ emission current for all measurements. The sputter crater diameter was $2 \mathrm{~mm}$. The angle between the surface normal and the Argon ion gun beam was $45^{\circ}$. The sputter procedure used was 1 time for $10 \mathrm{~s}, 1$ time for $60 \mathrm{~s}, 1$ time for $120 \mathrm{~s}$, and 1 time for $600 \mathrm{~s}$. The pressure within the analysis chamber was $10^{-7} \mathrm{mPa}$.

Automatic curve fitting was carried out using CasaXPS. A standard fit function GL(30) was chosen. Calibration of the binding energy (BE) of the measured spectra was performed by using the energy of the $\mathrm{C} 1 \mathrm{~s}$ peak (amorphous carbon at $\mathrm{BE}=284.5 \mathrm{eV}$ ) as an internal reference.

Table S6. Relative concentrations of the different B, N, C and O species in atomic\% determined via XPS analysis of the powdered precipitates and atomic ratios of boron to nitrogen and carbon to oxygen.

\begin{tabular}{|c|c|c|c|c|c|c|}
\hline \multirow{2}{*}{ Sample } & \multirow{2}{*}{$\begin{array}{c}\text { Species } \\
\text { and atomic } \\
\text { ratios }\end{array}$} & \multicolumn{5}{|c|}{ Overall sputter time / s } \\
\hline & & 0 & 10 & 70 & 190 & 790 \\
\hline \multirow{8}{*}{$\begin{array}{c}\text { HB dehy. with } \\
2 \text { mol\% of cat. } 2 \\
\text { up to } 1 \text { eq. } \mathrm{H}_{2} \\
\text { stored under argon }\end{array}$} & $\mathbf{O}$ & 8.32 & 8.13 & 7.84 & 7.37 & 6.99 \\
\hline & $\mathbf{N}\left(\mathrm{N}_{2} \mathrm{H}_{4}\right)$ & 0 & 0 & 0 & 0 & 0 \\
\hline & $\mathbf{N}(\mathrm{NBH})$ & 23.99 & 24.5 & 25.83 & 26.27 & 28.98 \\
\hline & C & 38.26 & 37.08 & 34.26 & 33.28 & 26.82 \\
\hline & $\mathbf{B}(\mathrm{BN})$ & 29.43 & 30.29 & 32.07 & 33.09 & 37.21 \\
\hline & $\mathbf{B}(\mathrm{BO})$ & 0 & 0 & 0 & 0 & 0 \\
\hline & $\mathrm{C}: \mathrm{O}^{\mathrm{a}}$ & 4.60 & 4.56 & 4.37 & 4.52 & 3.84 \\
\hline & B:N & 1.23 & 1.24 & 1.24 & 1.26 & 1.28 \\
\hline \multirow{8}{*}{$\begin{array}{l}\text { HB dehy. with } \\
2 \text { mol\% of cat. } 2 \\
\text { up to } 1 \text { eq. } \mathrm{H}_{2} \\
\text { stored at air }\end{array}$} & $\mathbf{O}$ & 13.73 & 13.29 & 13.33 & 13.24 & 12.84 \\
\hline & $\mathbf{N}\left(\mathrm{N}_{2} \mathrm{H}_{4}\right)$ & 15.9 & 13.44 & 11.08 & 7.44 & 7.76 \\
\hline & $\mathbf{N}(\mathrm{NBH})$ & 5.41 & 5.93 & 6.57 & 8.23 & 7.14 \\
\hline & C & 41.09 & 45.22 & 48.26 & 51.91 & 53.99 \\
\hline & $\mathbf{B}(\mathrm{BNH})$ & 16.18 & 14.53 & 13.29 & 12.51 & 11.86 \\
\hline & $\mathbf{B}(\mathrm{BO})$ & 7.69 & 7.59 & 7.47 & 6.67 & 6.40 \\
\hline & $\mathrm{C}: \mathrm{O}^{\mathrm{a}}$ & 2.99 & 3.40 & 3.62 & 3.92 & 4.20 \\
\hline & B:N & 1.12 & 1.14 & 1.18 & 1.22 & 1.23 \\
\hline
\end{tabular}

${ }^{\mathrm{a}}$ The $\mathrm{C}: \mathrm{O}$ ratio is expected to be close to four if the oxygen species only originates from solvent intercalation $\left(\mathrm{THF}, \mathrm{H}_{8} \mathrm{C}_{4} \mathrm{O}\right)$. 


\section{Raman spectroscopy}

The Raman spectrum was recorded using a LabRAM HR-800 spectrometer from Horiba Jobin Yvon equipped with a NdYAG laser at a wavelength of $532 \mathrm{~nm}$. A confocal microscope with 50-times magnification was applied in combination with a grid containing 1800 lines $/ \mathrm{mm}$. The sample was excited at ambient conditions for $60 \mathrm{~s}$ and 120 scans were acquired for signal averaging.

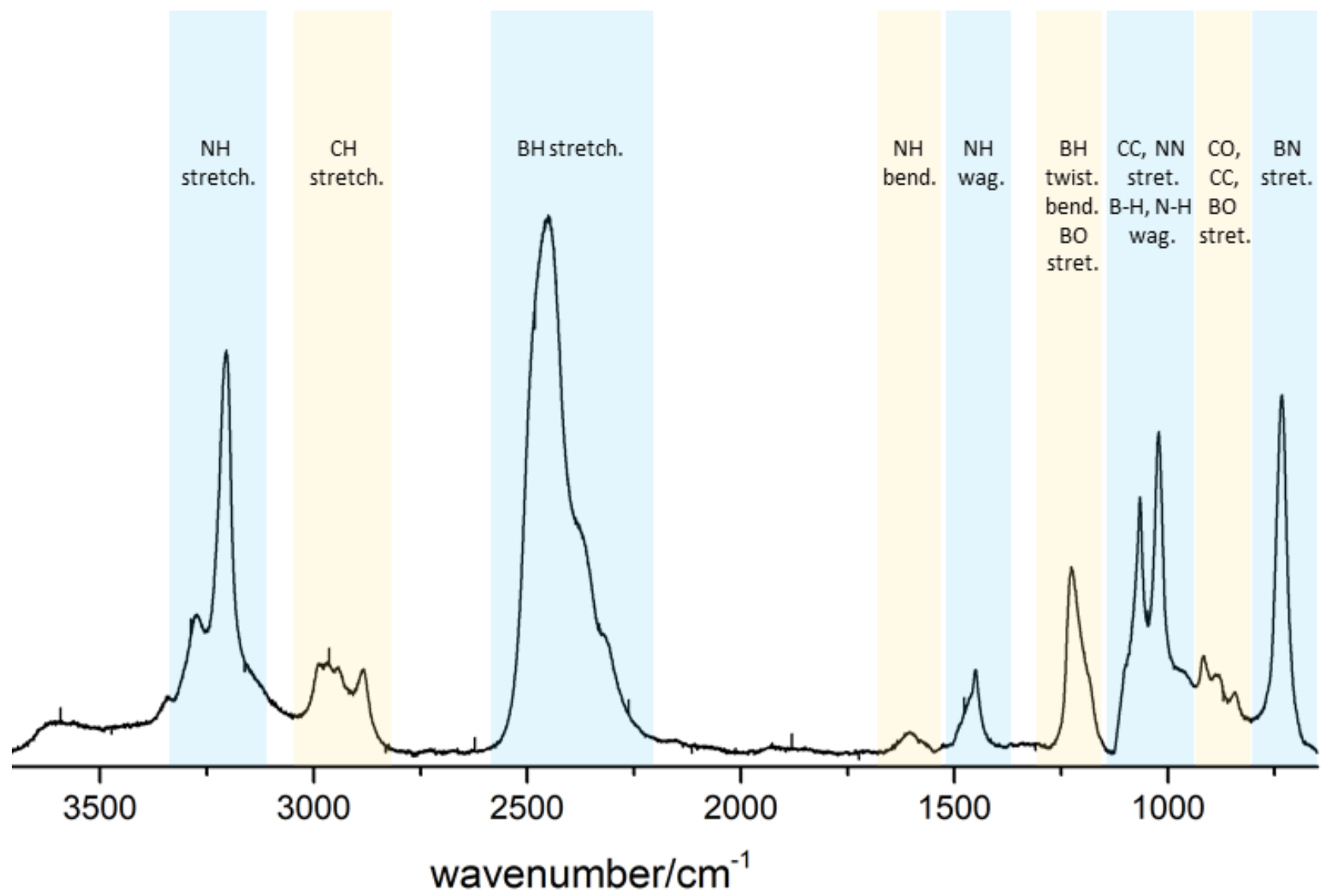

Figure S30. Raman spectrum of $\mathbf{H B}$ dehydrogenated up to an abstraction of 1.0 eq. $\mathrm{H}_{2}$ using 2 mol\% of catalyst 2 (stored in air for 2 month) and acquired at ambient conditions. Tentative assignment of all vibrations are based on literature data ${ }^{38,39,40,41,42,43}$ and depicted above the respective regions. 


\section{Validation of the combined NMR and DFT approach}

The structural characterization of the dehydrogenated material obtained from $\mathbf{H B}$ has been based on independent NMR experiments, DFT calculations, as well as elemental analysis. While the determination of the elemental composition from XPS and CHNS elemental analysis is straightforward, it is difficult to estimate the accuracy of calculated NMR parameters based on the gas-phase optimized model structures. The purpose of the following data is to justify this approach by comparison of experimentally determined and calculated NMR parameters for the literature known structure of poly(methylaminoborane), derived from dehydropolymerization of methylamine borane (MAB). ${ }^{44}$ The linear structure $\mathbf{S 1}$ is schematically depicted in Figure S31, which also includes NMR parameters that have been calculated for a short part of the linear polymer in analogy to the calculation of the model structures in the main text. The ${ }^{13} \mathrm{C}$ isotropic chemical shift offers an additional probe for the comparison of calculated and experimental data. In analogy to the calculated ${ }^{11} \mathrm{~B}$ and ${ }^{15} \mathrm{~N}$ isotropic chemical shifts (cf. Figure S27 and S28), the referencing has been performed via linear regression of experimentally determined chemical shifts from reference compounds reported in the literature plotted against calculated chemical shielding (see Figure S34).

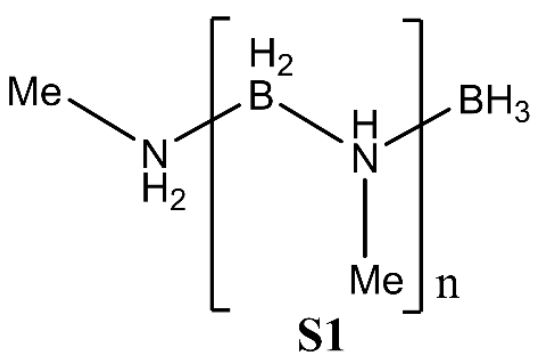

$\begin{aligned}{ }^{11} \mathrm{~B} \quad \delta_{\text {iso }}^{c s} & =-6.6 \mathrm{ppm} \\ C_{Q} & =1.65 \mathrm{MHz} \\ \eta_{Q} & =0.73 \\ { }^{13} \mathrm{C} \quad \delta_{\text {iso }}^{c S} & =37.2 \mathrm{ppm} \\ { }^{15} \mathrm{~N} \quad \delta_{\text {iso }}^{c S} & =-347 \mathrm{ppm}\end{aligned}$

Figure S31. Schematic representation of the linear polyaminoborane S1 obtained from abstraction of one equivalent $\mathrm{H}_{2}$ from methylamine borane. Additionally, ${ }^{11} \mathrm{~B},{ }^{13} \mathrm{C}$, and ${ }^{15} \mathrm{~N}$ NMR parameters calculated using DFT are listed.

Since the structure of the linear polymer is analogous to structure 16 (cf. Figure 6) similar NMR parameters were obtained for the ${ }^{11} \mathrm{~B}$ nuclei. To compare the calculated values to the experimental ones, ${ }^{11} \mathrm{~B}$ parameters have been extracted as demonstrated in the main text from ${ }^{11} \mathrm{~B}$ MAS and 3QMAS NMR spectra shown in Figures S31a and S31b, respectively. Compared to the results for the dehydrogenated hydrazine borane, similar spectra have been obtained containing a major and a minor ${ }^{11} \mathrm{~B}$ species. The ${ }^{11} \mathrm{~B}$ isotropic chemical shifts of these species are slightly higher than observed for dehydrogenated $\mathrm{HB}$, while the quadrupolar coupling constant is lower (cf. Table 1 and S6). The NMR parameters of the major ${ }^{11} \mathrm{~B}$ species are in good agreement with the DFT calculation as expected. While the experimentally determined and the calculated ${ }^{11} \mathrm{~B}$ isotropic chemical shifts are almost identical, the gas-phase calculated quadrupolar coupling constant is slightly larger than the experimentally determined one as has been similarly observed for the structures 20 and 21 assigned to dehydrogenated HB. However, the general good agreement between experiment and calculation for all discussed ${ }^{11} \mathrm{~B}$ NMR parameters clearly shows that structure determination based on DFT calculations on small model structures is a reasonable approach for the materials investigated in this study. 

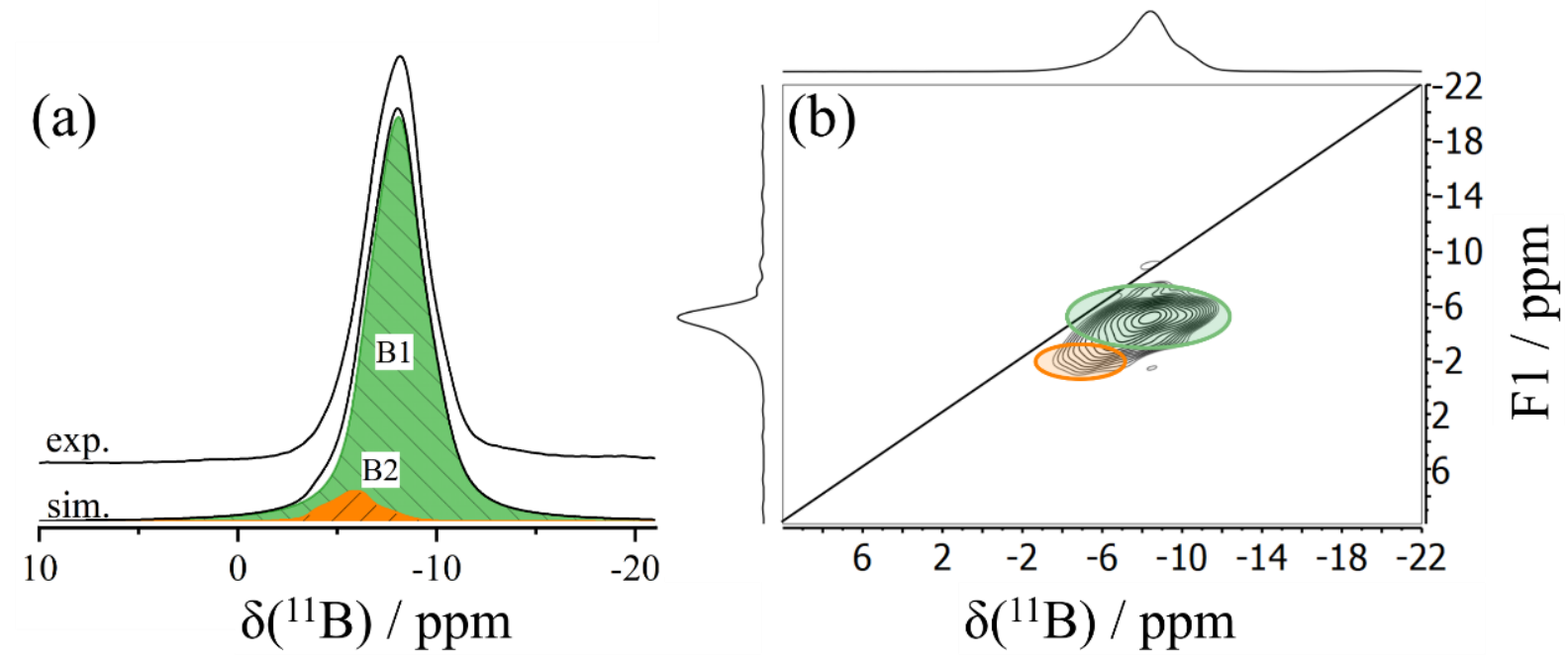

Figure S32. (a) Experimental ${ }^{11} \mathrm{~B}$ MAS NMR and (b) ${ }^{11} \mathrm{~B}$ 3QMAS NMR spectrum obtained for polyaminoborane $\mathbf{S 1}$ acquired at $11.74 \mathrm{~T}$ at a spinning frequency of $25 \mathrm{kHz}$ using ${ }^{1} \mathrm{H} \mathrm{SW}_{\mathrm{f}}-\mathrm{TPPM}$ decoupling. The dehydrogenated material was obtained after abstraction of one equivalent of hydrogen from MAB. The main component B1 (green) and the minor species B2 (orange) are highlighted in both spectra.

Table S7. Experimentally determined ${ }^{11} \mathrm{~B}$ chemical shift and quadrupolar coupling parameters for dehydrogenated methylamine borane $\mathbf{S 1}$ obtained by the release of one equivalent dihydrogen. The parameters result from line shape deconvolution of the ${ }^{11} \mathrm{~B}$ MAS spectrum and analysis of the center of gravity for species in the ${ }^{11} \mathrm{~B}$ 3QMAS spectrum in Figure S32.

\begin{tabular}{c||c|c|c|c|c|c|c}
\multicolumn{1}{c||}{} & \multicolumn{4}{c|}{${ }^{11} \mathrm{~B}$ MAS deconvolution } & \multicolumn{3}{c}{${ }^{11} \mathrm{~B}$ 3QMAS analysis } \\
$\begin{array}{c}{ }^{11} \mathrm{~B} \\
\text { species }\end{array}$ & $\begin{array}{c}\delta_{i s o}^{C S} \pm 0.5 \\
/ \mathrm{ppm}\end{array}$ & $\begin{array}{c}C_{Q} \pm 0.05 \\
/ \mathrm{MHz}\end{array}$ & $\begin{array}{c}\eta_{Q} \\
\pm 0.05\end{array}$ & $\begin{array}{c}\text { rel. int. } \\
/ \%\end{array}$ & $\begin{array}{c}\delta_{i s o}^{C S} \pm 0.5 \\
/ \mathrm{ppm}\end{array}$ & $\begin{array}{c}\text { SOQE } \pm 0.05 \\
/ \mathrm{MHz}\end{array}$ & $\begin{array}{c}C_{Q} \pm 0.05 \\
/ \mathrm{MHz}\end{array}$ \\
\hline \hline $\mathrm{B} 1$ & -6.1 & 1.34 & 0.7 & 94 & -6.3 & 1.47 & 1.36 \\
$\mathrm{~B} 2$ & -3.6 & 1.41 & 0.7 & 6 & -3.2 & 1.58 & 1.42 \\
\hline
\end{tabular}

Furthermore the ${ }^{13} \mathrm{C}$ isotropic chemical shift of the methyl group can be used as an additional probe to test the accuracy of the DFT-calculated NMR parameters of such amorphous compounds. In Figure S33a, the ${ }^{13} \mathrm{C}\left\{{ }^{1} \mathrm{H}\right\}$ CPMAS NMR spectrum acquired on dehydrogenated methylamine borane is depicted, from which an ${ }^{13} \mathrm{C}$ isotropic chemical shift of $36.2 \mathrm{ppm}$ has been extracted. Considering the comparatively large chemical shift range of ${ }^{13} \mathrm{C}$ nuclei in general, this value is in an excellent agreement with the calculated parameter of $37.2 \mathrm{ppm}$. This indicates that the calculated ${ }^{13} \mathrm{C}$ shifts might be used as well as the ${ }^{11} \mathrm{~B}$ parameters for the DFT-aided structure assignment of unknown carbon-containing BNH networks. In addition to the main species, the ${ }^{13} \mathrm{C}\left\{{ }^{1} \mathrm{H}\right\}$ CPMAS NMR spectrum contains a minor signal at about $20 \mathrm{ppm}$ which contributes approximately $4 \%$ to the overall signal intensity. Even though the CPMAS experiment is not quantitative, it is reasonable to attribute this resonance to ${ }^{13} \mathrm{C}$ nuclei at the end of the polymer chain. This can be confirmed via DFT calculation of the $\delta_{\text {iso }}^{C S}\left({ }^{13} \mathrm{C}\right)$ for the $\mathrm{NH}_{2} \mathrm{CH}_{3}$ group attached to the terminal boron atom in Figure S30, which leads to a ${ }^{13} \mathrm{C}$ isotropic chemical shift of $26 \mathrm{ppm}$. The larger deviation from the experimental value compared to the main component can likely be attributed to the weak representation of such terminal groups in gas-phase optimized structures. 
Finally, we performed an analogous analysis of the ${ }^{15} \mathrm{~N}$ isotropic chemical shift, which in the main text has been assumed to be less suitable for a structural assignment. The ${ }^{15} \mathrm{~N}\left\{{ }^{1} \mathrm{H}\right\}$ CPMAS NMR spectrum of dehydrogenated MAB is depicted in Figure $\mathrm{S} 33 \mathrm{~b}$ showing a single resonance at an ${ }^{15} \mathrm{~N}$ isotropic chemical shift of $-365 \mathrm{ppm}$. This value differs significantly from the calculated one by $22 \mathrm{ppm}$. A similar deviation has been obtained for the calculated ${ }^{15} \mathrm{~N}$ isotropic chemical shifts of structures $\mathbf{2 0}$ and $\mathbf{2 1}$ compared to the experimental value of dehydrogenated $\mathrm{HB}$.
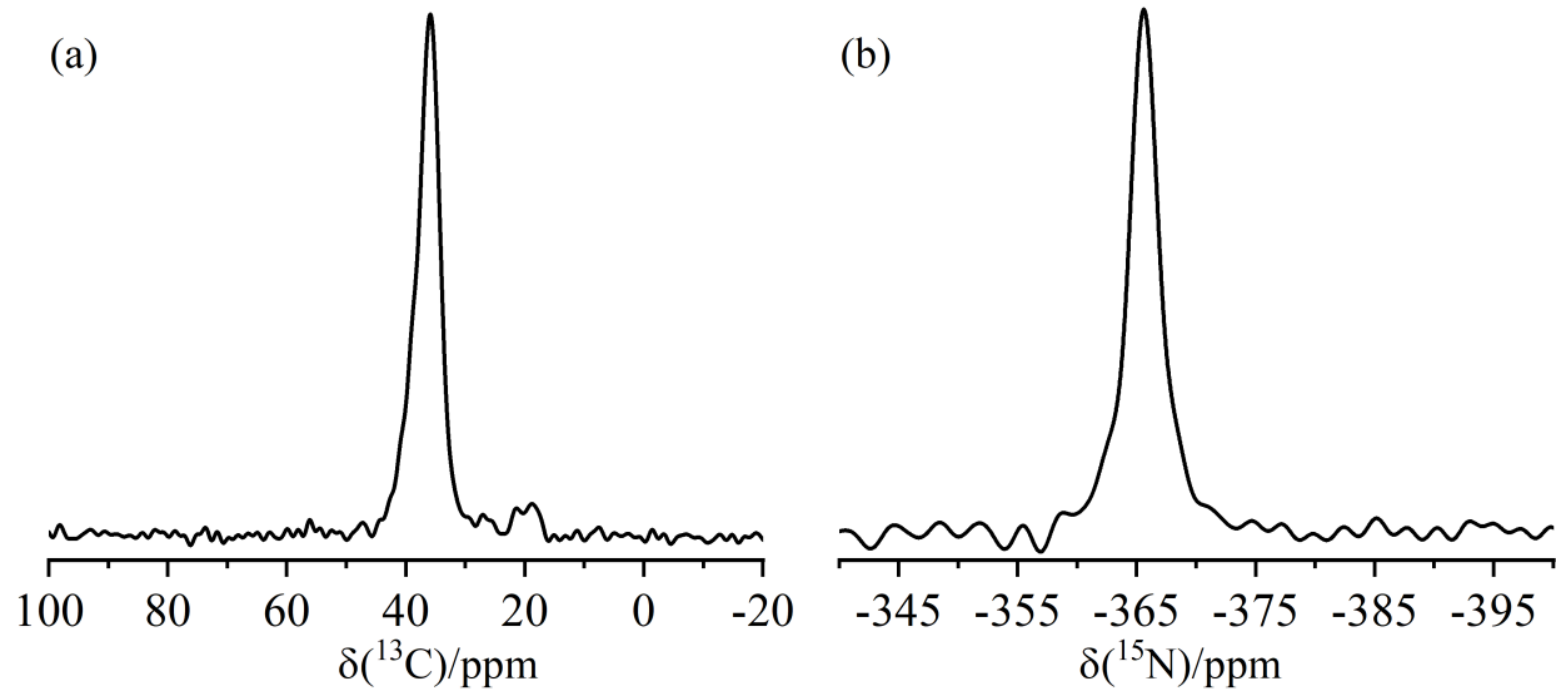

Figure S33. (a) Experimental ${ }^{13} \mathrm{C}\left\{{ }^{1} \mathrm{H}\right\}$ CPMAS NMR spectrum acquired at $11.74 \mathrm{~T}$ at a spinning frequency of $25 \mathrm{kHz}$ and (b) ${ }^{15} \mathrm{~N}\left\{{ }^{1} \mathrm{H}\right\}$ CPMAS NMR spectrum acquired at $7.05 \mathrm{~T}$ at a spinning frequency of $10 \mathrm{kHz}$, both obtained for the dehydrogenated MAB compound $\mathbf{S 1}$ using ${ }^{1} \mathrm{H} \mathrm{SW}_{\mathrm{f}}$-TPPM decoupling during acquisition. The dehydrogenated material was received after abstraction of one equivalent $\mathrm{H}_{2}$.

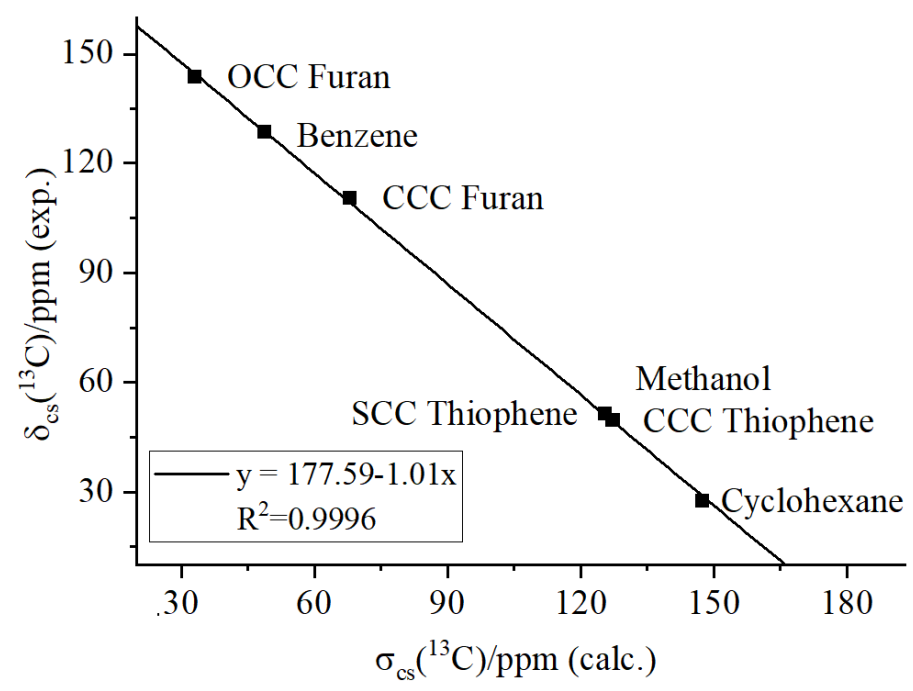

Figure S34. The experimentally determined ${ }^{13} \mathrm{C}$ isotropic chemical shifts ${ }^{45}$ of reference structures plotted against calculated chemical shielding. Additionally, the function resulting from linear regression is depicted. 


\section{References}

1 Alberico, E.; Sponholz, P.; Cordes, C.; Nielsen, M.; Drexler, H.-J.; Baumann, W.; Junge, H.; Beller, M. Selective Hydrogen Production from Methanol with a Defined Iron Pincer Catalyst under Mild Conditions. Angew. Chem. Int. Ed. 2013, 52, 14162-14166.

2 Chakraborty, S.; Brennessel, W. W.; Jones, W. D. A Molecular Iron Catalyst for the Acceptorless Dehydrogenation and Hydrogenation of N-Heterocycles. J. Am. Chem. Soc. 2014, 136, 8564-8567.

3 Moury, R.; Moussa, G.; Demirci, U. B.; Hannauer, J.; Bernard, S.; Petit, E.; van der Lee, A.; Miele, P. Hydrazine Borane: Synthesis, Characterization, and Application Prospects in Chemical Hydrogen Storage. Phys. Chem. Chem. Phys. 2012, 14, 1768-1777.

4 Beweries, T.; Thomas, J.; Klahn, M.; Schulz, A.; Heller D.; Rosenthal, U. Catalytic and Kinetic Studies of the Dehydrogenation of Dimethylamine Borane with an $i \operatorname{Pr}$ Substituted Titanocene Catalyst. ChemCatChem 2011, 3, 1865-1868.

5 Drexler, H.-J.; Preetz, A.; Schmidt, T.; Heller, D. in The Handbook of Homogeneous Hydrogenation, Vol. 1 (Eds.: de Vries, J. G.; Elsevier, C. J.), Wiley-VCH, Weinheim, 2007, pp. 257.

6 Jiao, H.; Junge, K.; Alberico, E.; Beller, M. A Comparative Computationally Study About the Defined M(II) Pincer Hydrogenation Catalysts (M=Fe, Ru, Os). J. Comput. Chem. 2016, 37, 168176.

7 (a) Alberico, E.; Lennox, A. J.; Vogt, L. K.; Jiao, H.; Baumann, W.; Drexler, H.-J.; Nielsen, M.; Spannenberg, A.; Checinski, M. P.; Junge, H.; Beller, M. Unravelling the Mechanism of Basic Aqueous Methanol Dehydrogenation Catalyzed by Ru-PNP Pincer Complexes. J. Am. Chem. Soc. 2016, 138, 14890-14904; b) Wei, Z.; Junge, K.; Beller, M.; Jiao, H. Hydrogenation of Phenylsubstituted $\mathrm{C} \equiv \mathrm{N}, \mathrm{C}=\mathrm{N}, \mathrm{C} \equiv \mathrm{C}, \mathrm{C}=\mathrm{C}$ and $\mathrm{C}=\mathrm{O}$ Functional Groups by $\mathrm{Cr}$, Mo and $\mathrm{W}$ PNP Pincer Complexes - A DFT Study Catal. Sci. Technol. 2017, 7, 2298-2307.

8 Perdew, J. P. Density-Functional Approximation for the Correlation Energy of the Inhomogeneous Electron Gas. Phys. Rev. B 1986, 33, 8822-8824.

9 Schaefer, A.; Huber, C.; Ahlrichs, R. Fully Optimized Contracted Gaussian Basis Sets of Triple Zeta Valence Quality for Atoms Li to Kr. J. Chem. Phys. 1994, 100, 5829-5835.

10 Gaussian 09, Revision C.01, M. J. Frisch, G. W. Trucks, H. B. Schlegel, G. E. Scuseria, M. A. Robb, J. R. Cheeseman, G. Scalmani, V. Barone, B. Mennucci, G. A. Petersson, H. Nakatsuji, M. Caricato, X. Li, H. P. Hratchian, A. F. Izmaylov, J. Bloino, G. Zheng, J. L. Sonnenberg, M. Hada, M. Ehara, K. Toyota, R. Fukuda, J. Hasegawa, M. Ishida, T. Nakajima, Y. Honda, O. Kitao, H. Nakai, T. Vreven, J. A. Montgomery, Jr., J. E. Peralta, F. Ogliaro, M. Bearpark, J. J. Heyd, E. Brothers, K. N. Kudin, V. N. Staroverov, T. Keith, R. Kobayashi, J. Normand, K. Raghavachari, A. Rendell, J. C. Burant, S. S. Iyengar, J. Tomasi, M. Cossi, N. Rega, J. M. Millam, M. Klene, J. E. Knox, J. B. Cross, V. Bakken, C. Adamo, J. Jaramillo, R. Gomperts, R. E. Stratmann, O. Yazyev, A. J. Austin, R. Cammi, C. Pomelli, J. W. Ochterski, R. L. Martin, K. Morokuma, V. G. Zakrzewski, G. A. Voth, P. Salvador, J. J. Dannenberg, S. Dapprich, A. D. Daniels, O. Farkas, J. B. Foresman, J. V. Ortiz, J. Cioslowski, and D. J. Fox, Gaussian, Inc., Wallingford CT, 2010

11 Marenich, A. V.; Cramer, C. J.; Truhlar, D. G. Universal solvation model based on solute electron density and a continuum model of the solvent defined by the bulk dielectric constant and atomic surface tensions. J. Phys. Chem. B 2009, 113, 6378-6396.

12 Grimme, S.; Ehrlich, S.; Goerigk, L. Effect of the damping function in dispersion corrected density functional theory. J. Comp. Chem. 2011, 32, 1456-65 
13 Xia, T.; Wei, Z.; Spiegelberg, B.; Jiao, H.; Hinze, S.; de Vries, J. G. Isomerization of Allylic Alcohols to Ketones Catalyzed by Well-Defined Iron PNP Pincer Catalysts. Chem. Eur. J. 2018, 24, 4043-4049.

14 a) Yang, X. A Self-Promotion Mechanism for Efficient Dehydrogenation of Ethanol Catalyzed by Pincer Ruthenium and Iron Complexes: Aliphatic versus Aromatic Ligands. ACS Catal. 2013, 3, 2684-2688. b) Qu, S.; Dai, H.; Dang, Y.; Wang, Z.-X.; Guan, H. Computational Mechanistic Study of Fe-Catalyzed Hydrogenation of Esters to Alcohols: Improving Catalysis by Accelerating Precatalyst Activation with a Lewis Base. ACS Catal. 2014, 4, 4377-4388.

15 Budweg, S.; Wei, Z.; Jiao, H.; Junge, K.; Beller, M. Iron PNP-Pincer-catalyzed TransferDehydrogenation of Secondary Alcohols. ChemSusChem 2019, 12, in press.

16 a) Wei, Z.; Jiao, H. Bifunctional aliphatic PNP pincer catalysts for hydrogenation: Mechanisms and scope. Adv. Inorg. Chem. 2019, 73, 323-384; b) Wei, Z.; de Aguirre, A.; Junge, K.; Beller, M.; Jiao, H. Benyzl Alcohol Dehydrogenative Coupling Catalyzed by Defined Mn and Re PNP Pincer Complexes - A Computational Mechanistic Study. Eur. J. Inorg. Chem. 2018, 2018, 4643-4657; c) Wei, Z.; de Aguirre, A.; Junge, K.; Beller, M.; Jiao, H. Exploring the mechanisms of aqueous methanol dehydrogenation catalyzed by defined PNP Mn and Re pincer complexes under base-free as well as strong base conditions. Catal. Sci. Technol. 2018, 8, 3649-3665; d) Alberico, E.; Lennox, A. J. J.; Vogt, L. K.; Jiao, H.; Baumann, W.; Drexler, H.-J.; Nielsen, M.; Spannenberg, A.; Checinski, M. P.; Junge, H.; Beller, M. Unravelling the Mechanism of Basic Aqueous Methanol Dehydrogenation Catalyzed by Ru-PNP Pincer Complexes. J. Am. Chem. Soc. 2016, 138, 1489014904; e) Jiao, H.; Junge, K.; Alberico, E.; Beller, M. A comparative computationally study about the defined $\mathrm{m}$ (II) pincer hydrogenation catalysts $(\mathrm{m}=\mathrm{fe}$, ru, os). J. Comput. Chem. 2016, 37, 168176.

17 Glüer, A.; Förster, M.; Celinski, V. R.; Schmedt auf der Günne, J.; Holthausen, M. C.; Schneider, S. Highly Active Iron Catalyst for Ammonia Borane Dehydrocoupling at Room Temperature. ACS Catalysis 2015, 7214-7217.

18 Amoureux, J. P.; Fernandez, C.; Steuernagel, S. Z Filtering in MQMAS NMR. J. Magn. Reson Ser. A 1996, 123, 116.

19 Massiot, D.; Fayon, F.; Capron, M.; King, I.; Le Calvé, S.; Alonso, B.; Durand, J.-O.; Bujoli, B.; Gan, Z.; Hoatson, G. Modelling One-and Two-Dimensional Solid-State NMR spectra. Magn. Res. Chem. 2001, 40, 70-76.

20 Jmol: an open-source Java viewer for chemical structures in 3D. http://www.jmol.org/

21 Tao, J.; Perdew, J. P.; Staroverov, V. N.; Scuseria, G. E. Climbing the Density Functional Ladder: Nonempirical Meta-Generalized Gradient Approximation Designed for Molecules and Solids. Phys. Rev. Lett. 2003, 91, 146401.

22 Becke, A. D. Density-Functional Thermochemistry. III. The Role of Exact Exchange. J. Chem. Phys. 1993, 98, 5648-5652.

23 Stephens, P. J.; Devlin, F. J.; Chabalowski, C. F.; Frisch, M. J. Ab Initio Calculation of Vibrational Absorption and Circular Dichroism Spectra Using Density Functional Force Fields. J. Phys. Chem. 1994, 98, 11623-11627.

24 Ahlrichs, R.; Bär, M.; Häser, M.; Horn, H.; Kölmel, C. Electronic Structure Calculations on Workstation Computers: The Program System Turbomole. Chem. Phys. Lett. 1989, 162, 165-169. 
26 Weigend, F.; Ahlrichs, R. Balanced Basis Sets of Split Valence, Triple Zeta Valence and Quadruple Zeta Valence Quality for H to Rn: Design and assessment of Accuracy. Phys. Chem. Chem. Phys. 2005, 7, 3297-3305.

27 Gaussian 09, Revision A.02, M. J. Frisch et al., Gaussian, Inc., Wallingford CT, 2016

28 Grimme, S. Semiempirical GGA-Type Density Functional Constructed with a Long-Range Dispersion Correction. J. Comput. Chem .2006, 27, 1787-1799.

29 Feller, D. The Role of Databases in Support of Computational Chemistry Calculations. J. Comput. Chem. 1996, 17, 1571-1586.

30 Schuchardt, K. L.; Didier, B. T.; Elsethagen, T.; Sun, L.; Gurumoorthi, V.; Chase, J.; Li, J.; Windus, T. L. Basis Set Exchange: A Community Database for Computational Sciences. J. Chem. Inf. Model. 2007, 47, 1045-52.

31 Woon, D. E.; Dunning, T. H. Gaussian Basis Sets for Use in Correlated Molecular Calculations. V. Core-Valence Basis Sets for Boron Through Neon. J. Chem. Phys. 1995, 103, 4572-4585.

32 Peterson, K. A.; Dunning, T. H. Accurate Correlation Consistent Basis Sets for Molecular CoreValence Correlation Effects: The Second Row Atoms Al-Ar, and the First Row Atoms B-Ne Revisited. J. Chem. Phys. 2002, 117, 10548-10560.

33 a) Stowe, A. C.; Shaw, W. J.; Linehan, J. C.; Schmid, B.; Autrey, T. In Situ Solid State ${ }^{11}$ B MASNMR Studies of the Thermal Decomposition of Ammonia Borane: Mechanistic Studies of the Hydrogen Release Pathways From a Solid State Hydrogen Storage Material. Phys. Chem. Chem. Phys., 2007, 9, 1831-1836; b) Yot, P. G.; Yadav, V.; Ould Amara, S.; Itiè, J.-P.; Demirci, U. B.; Maurin, G. Unraveling the Mechanical Behaviour of Hydrazine Borane $\left(\mathrm{NH}_{2}-\mathrm{NH}_{2}-\mathrm{BH}_{3}\right)$. Phys. Chem. Chem. Phys., 2018, 20, 2845-2850; c) Sethio, D.; Daku, L. M.; Hagemann, H. A Theoretical Study of the Spectroscopic Properties of $\mathrm{B}_{2} \mathrm{H}_{6}$ and of a Series of $\mathrm{B}_{x} \mathrm{H}_{\mathrm{y}}{ }^{{ }^{-}}$species $(x=1-12, y=3-14$, $z=0-2)$ : From $\mathrm{BH}_{3}$ to $\mathrm{B}_{12} \mathrm{H}^{122-}$. Int. J. Hydrogen Energy, 2016, 41, 6814-6824.

34 Li, J.-s.; Zhang, C.-r.; Li, B.; Cao, F.; Wang, S.-q. An Investigation on the Synthesis of Borazine. Inorg. Chim. Acta 2011, 366, 173-176.

35 Gunaydin-Sen, O.; Achey, R.; Dalal, N. S.; Stowe, A.; Autrey, T. High Resolution ${ }^{15}$ N NMR of the $225 \mathrm{~K}$ Phase Transition of Ammonia Borane $\left(\mathrm{NH}_{3} \mathrm{BH}_{3}\right)$ : Mixed Order-Disorder and Displacive Behavior. J. Phys. Chem. B 2007, 111, 677-681.

36 Bertani, P.; Raya, J.; Bechinger, B. ${ }^{15} \mathrm{~N}$ Chemical Shift Referencing in Solid State NMR. Solid State Nucl. Mag. 2014, 61-62, 15-18.

37 Städeli, W.; von Philipsborn, W. ${ }^{15} \mathrm{~N}$ NMR Substituent Effects in Pyridines and Pyrimidines. Organic Magnetic Resonance 1981, 15, 106-109.

38 Durig, J. R.; Bush, S. F.; Mercer, E. E. Vibrational Spectrum of Hydrazine- $d_{4}$ and a Raman Study of Hydrogen Bonding in Hydrazine. J. Chem. Phys. 1966, 44, 4238-4247.

39 Pylypko, S.; Petit, E.; Yot, P. G.; Salles, F.; Cretin, M.; Miele, P.; Demirci, U. B. Key Study on the Potential of Hydrazine Bisborane for Solid- and Liquid-State Chemical Hydrogen Storage. Inorg. Chem. 2015, 54, 4574-4583.

40 Hanousek, F.; Haruda, F. Infrared and Raman Spectra of Hydrazine-bis(trihydroborane). Collect. Czechoslovak. Chem. Commun. 1973, 38, 2692-2696.

41 Ziparo, C.; Colognesi, D.; Giannasi, A.; Zoppi, M. Raman Spectra of Ammonia Borane: Low Frequency Lattice Modes. J. Phys. Chem. A 2012, 116, 8827-8832.

42 Takasu, Y.; Matsumoto, S.; Fujii, Y.; Nishio, I. Raman Study of the Low Temperature Behavior of Tetrahydrofuran Molecule in the Cage of Clathrate Hydrate. Chem. Phys. Lett. 2015, 627, 39-43. 
43 Jun, L.; Shuping, X.; Shiyang, G. FT-IR and Raman Spectroscopic Study of Hydrated Borates. Spectrochim. Acta A: Mol. Biomol. Spectr. 1995, 51, 519-532.

44 a) Anke, F.; Han, D.; Klahn, M.; Spannenberg, A.; Beweries, T. Formation of high-molecular weight polyaminoborane by Fe hydride catalysed dehydrocoupling of methylamine borane. Dalton Trans. 2017, 46, 6843-6847; b) Staubitz, A.; Soto, A. P.; Manners, I. Iridium-Catalyzed Dehydrocoupling of Primary Amine-Borane Adducts: A Route to High Molecular Weight Polyaminoboranes, Boron-Nitrogen Analogues of Polyolefins. Angew. Chem., Int. Ed. 2008, 47, 6212-6215.

45 a) Gottlieb, H. E.; Kotlyar, V.; Nudelman, A. NMR Chemical Shifts of Common Laboratory Solvents as Trace Impurities. J. Org. Chem. 1997, 62, 7512-7515; b) Block, E.; Birringer, M.; DeOrazio, R.; Fabian, J.; Glass, R. S.; Guo, C.; He, C.; Lorance, E.; Qian, Q.; Schroeder, T. B.; Shan, Z.; Thiruvazhi, M.; Wilson, G. S.; Zhang, X. Synthesis, Properties, Oxidation, and Electrochemistry of 1,2-Dichalcogenins. J. Am. Chem. Soc. 2000, 122, 5052-5064; c) AlvarezIbarra, C.; L. Quiroga-Feijóo, M.; Toledano, E. An analysis of substituent effects on ${ }^{1} \mathrm{H}$ and ${ }^{13} \mathrm{C}$ NMR parameters of substituted furans. Linear free energy relationships and PM3 semiempirical calculations. J. Chem. Soc., Perkin Trans. 2 1998, 679-690. 